\title{
INTRODUCTION
}

\section{FENG YOULAN'S WORK OF A CENTURY}

\author{
Diane B. Obenchain, Editor and Translator
}

Part One: Openings

Many working in the field of Chinese philosophy today began by tracking with Feng Youlan ${ }^{1}$ through his well-known two-volume $A$ History of Chinese Philosophy, published in Chinese in 1930 and 1934 and in English in 1952.3.2 For greater audiences of Chinese and nonChinese alike, Feng's $A$ History of Chinese Philosophy opened doors and windows into the walled-in heritage of Chinese philosophical thought that had been accessed by privileged few for most of the Middle Kingdom's three-thousand years of recorded history. Being one of few sources of its kind, ${ }^{3}$ particularly in English, it was along with Feng Youlan, as he penetrated through the issues of Chinese philosophical thought for himself in these volumes, that those keen to learn more of China's philosophical past began their engagement in the long, ceasless hard work of penetrating through Chinese philosophy for themselves as well. And, having in time moved on to first-hand, direct study of the Chinese primary sources Feng Youlan himself had used, many, who continue to track the path of Chinese philosophy today, keep a copy of Feng's work near to hand, an honest and reliable friend on the way.

This action in itself gives great tribute to one whom many assess as the greatest philosophical thinker of the Chinese twentieth century.

On November 26, 1990, Professor Feng Youlan passed on at almost the age of ninety-five. Many had already made travel arrangements to 
attend an "International Seminar on the Thought of Feng Youlan" to be held in Beijing during the first week of December, at which time we planned to celebrate with him his ninety-fifth birthday on December 4 . Most carried through with those plans once the news of his passing was known: we came together to commemorate a lifetime of contribution to China, to the Chinese people.

Feng's ninety-five years spanned the whole of the great organismicshaking of China's twentieth century that cried out, and, indeed, at times seemed utterly destined, to change everything, from start to finish, to wipe the slate clean, to begin again. Considerable transposing in the expression of Feng's own views there has been as he, alongside others, was taken up into the upheaval and through it endeavored to discern and to track the way of Chinese philosophical thinking down through the ages while at the same time to step forth into a "future unknown."

As the twentieth century gathered momentum, more voices around the world from West Asian deserts, to the illo tempore river's edge of the Ganges, to China's Mount Tai, have added volume to an initially dischordant but increasingly appreciated two-part harmony of "change what must be changed, but hold on to what is true." It is a strain we hear not only in the Middle East, in India, in China, but, indeed, all over the world, in North and Scuth America, in Europe, in Africa - everywhere. As China, along with others, carries on with new variations of its continuing song, we have been priviledged to come alongside and listen to the piping of one of its transmitters, Feng Youlan, who, along with important others, gave himself to the responsibility, the calling (ming), of handing down to generations yet to come China's distinct melody: retuning it to harmonize with new melodies coming from abroad, while yet not abandoning the old instruments.

This small collection of papers here presented in tribute to Professor Feng Youlan does not attempt to explain and to interpret in detail the whole of Feng's subtle and multi-faceted thinking nor those events, particularly political, that pressed upon his life and thought. Analysis, whole and fine, of the times and philosophy of Feng Youlan will surely engage 
the lifetime work of more than one scholar. ${ }^{5}$ To such efforts perhaps this small collection of translated philosophical essays from the commemorative conference within days of Feng's passing may serve as a resource of collected vision for investigating and assessing Feng Youlan's contributions to Chinese philosophy as well as to contemporary world philosophy. In addition, having gathered with others during the first week of December 1990 to attend the "International Seminar on the Thought of Feng Youlan," one's purpose here is also to share with those who were not able to attend the moment of Feng's passing and what it meant at the time to his colleagues, family, and friends.

The mood of the "International Seminar on the Thought of Feng Youlan" was complex. On the one hand, there were the reverential $l i$ (ceremonial rites) of mourning and gratitude. On the other hand, there were the often times heated and intense debates about what Feng Youlan actually said, what he truly meant, and what difference it all makes to the direction Chinese philosophy is going now. In presenting images and sounds from both sets of events, this Editor and translator seeks to let voices of others do most of the talking. This is to emphasize that Professor Feng's life and work, significantly and differently, touched many.

A documentary film began the conference on the morning of December 4. Professor Feng is present throughout the film and for those who knew him, it was warming to see him still thinking, exploring, expounding. ${ }^{6}$ The film in part presents author Zong Pu, ${ }^{7}$ Feng Youlan's daughter, narrating poignent moments with her father. Zong Pu was with her father through the end of his days at Beijing University. With this palpable closeness to Professor Feng in heart-mind, we moved from the film presented during the opening ceremonies in the morning to the "gaobie (saying goodbye)" ceremonies which took place in the afternoon.

A translation of excerpts of the documentary film and a remembrance of the "gaobie" ceremonies as recalled through the eyes of Mr. Thanh Van Tran comprise "Section One: Perspectives" of this volume. In addition, Mr. Thanh in his essay reviews main issues spanning seven 
decades of Feng's philosophical work and takes one into the heart of what matters in Feng's philosophy. In so doing, Mr. Thanh offers further orientation to what follows in this volume.

The second and third days of the conference were given to plenary and small group discussions of papers. The papers delivered in plenary session "put on the board," as it were, the topics and range of subject matter which were taken up in small group discussion. The papers offered in this volume are a selection of papers from both plenary and small discussion sessions. The papers are largely those of members of the International Academy of Chinese Culture (Zhongguo Wenhua Shuyuan) ${ }^{8}$ which, along with some participation by members of the Chinese Confucius Society, sponsored the conference. ${ }^{9}$ Translations of the papers and of the documentary film are those of the Editor who takes responsibility for any misrepresentation contained therein. ${ }^{10}$

Translation of papers in this volume is intended for readers of Feng Youlan's work in both English and Chinese. Translation of papers herein is also to assist those starting in the field of Chinese philosophy as well as to provide further depth of detail for those advanced in the field. Because Feng's A History of Chinese Philosophy, whether in long or short version, is one work with which Western students often begin their study of Chinese philosophy or, at least, is one work to which students turn frequently for reference, undergraduates and graduates engaged in the study of Chinese philosophy in English may find these translations helpful as a kind of companion volume, lending perspective to and critique of Feng's thinking.

Given the range of readers to whom this translation is addressed and given the range of issues to which Feng Youlan's philosophy gives expression, we have thought it helpful to provide an Introduction that is more detailed than is the usual case with collected papers of a conference. As a collection of translated papers, with an effort to be consistent in use of translated English terms throughout the volume, in this Introduction we seek to introduce not only the conference and overall concerns of this volume (Part One), significant events in the life of Feng Youlan 
and the essays of the conference as they speak to these events (Part Two), but also the method of translation which we have used and reasons for specific choices in English of terms which can convey consistently the subtlety of both Feng Youlan's thinking and his critics (Part Three).

One problem in translation work of this kind is that Chinese-English dictionaries or dictionaries between any two languages are, in practice, matching manuals. Each term of any language is shaped and colored by its relationship with other terms in that language, with the whole of a language being structured by certain principled discernments of the world, on the part of a group of people speaking that language. Expression of these principled discernments changes over time as individual persons see more in and add more to the terms of a language. Hence, one word in one language rarely, if ever, means exactly what it is matched with in another language. Matchings of meanings may come very close or they may not. Some matchings may be literal; some may be notional.

A second problem in translation work of this kind is that, on the one hand, Chinese philosophical terms are used most often with traditional and customary Chinese meanings and patterns. On the other hand, these same Chinese terms are increasingly used to translate into Chinese certain prominent Western philosophical notions. Deciding between these two types of usage when translating from Chinese into English of ten becomes subtly complex and difficult, especially when working with twentieth century thinkers such as Feng Youlan, who was extremely well-read in both Chinese philosophy and Western philosophy.

We introduce two examples of these two translation problems here. These examples and others will be explored further in Part Three of this Introduction.

First, Chinese $l i$ has been matched with English "pattern," when a more visual image of its meaning is preferred, and with English "principle," when a more abstract sense of its meaning is preferred. ${ }^{11}$ We have put both together in our usual rendering of the matching as "li" (pattern, principle). Going the other way round, that is, when translating some English or Western philosophical terms into Chinese, it has 
become standard to use Chinese $l i$ in the matching process. For example, English "rational" is rendered in Chinese as lixing de, meaning literally in Chinese "li-natured." English "reasonable" is rendered as lizhi de, meaning literally in Chinese "of the wisdom of li." English "idea" is matched with Chinese linian, literally "recitation of li." English "theory" is Chinese lilun, literally "discussion of $l i$." All of these matches from English to Chinese build upon a, perhaps, unconscious yet fundamentally assumed, match between Plato's "idea" or Aristotle's "eidos" (form) and Chinese $l i$, from which all the other matches just listed are derived. Feng Youlan himself assumed and, indeed, argued for this very fundamental match.

A problem arises, however, when one who is thinking and writing in Chinese wants to question whether this match of Plato's idea and Chinese $l i$ is as close as philosophers have assumed. How is one going to talk about the problem in Chinese? What vocabulary will one use? For example, if, in Chinese, one asks whether $l i$ is rational, given the EnglishChinese match between "rational" and lixing, one ends up asking whether $l i$ is lixing de. Literally, this is to ask whether $l i$ is "li-natured," which, to say the least, is redundant! The same is true when asking in Chinese whether $l i$ is reasonable: one ends up asking whether $l i$ is lizhi de, which is literally to ask if $l i$ is "of the wisdom of $l i$." So also with the fundamental match of $l i$ and "idea": one ends up asking in Chinese whether $l i$ is "recitation of $l i$ " (linian). In other words, in these and other examples of English philosophical terms matched with Chinese terms using $l i$, if we want to question the match between $l i$ and "idea," it is difficult to do so in contemporary Chinese without using Chinese terms which assume the very match which we wish to question. In the end, we are not able to ask anything of what we want to ask comparatively.

The problem seems not to occur as often the other way round, that is, when using Western or English terms to translate Chinese terms, although occur the problem does, as we shall see when we turn to our second example below. In recent times, good effort has been made not to substitute, or at least not so patly, one Western philosophical term for 
one Chinese philosophical term. Instead, having made careful linguistic as well as philosophical exploration of a Chinese philosophical term, English romanization of the Chinese term is offered first, followed by one or more suggested English matches put in parentheses next to it. This method allows the Chinese term to speak, as much as possible, on its own rather than through and, possibly distorted by, English substitutes for it. It is this method which we endeavor to employ throughout this volume. ${ }^{12}$

Of course, Chinese philosophers will say that they know very well when an instance of Chinese $l i$ in combination with another Chinese term is a Chinese fabricated term for translating English "rational" or English "reasonable" and that they do not ever take the fabricated term literally. As true as this may be, still this practice does allow for some casual glossing over assumed matches between terms that many today want to re-explore. Is Chinese $l i$ a thorough-going or complete match for Western "idea" or vice versa? Where are the similarities? Where are the differences? Getting at these similarities and differences while thinking and writing in Chinese may well require a new Chinese approach to handling Western terms, so as to avoid unexplored assumptions confounding the very investigation one wants to make.

This same problem comes up even more subtly in the translation of Chinese "you" meaning literally "to have" or "having." which is not a fabricated Chinese term but which, nonetheless, is used to translate, indeed, "substitute for," Western philosophical notions of "there exists/ there is," "existence," and "Being." Again, like Chinese li (pattern, principle), Chinese you (have, having) has its own philosophical meaning or meanings long within the Chinese philosophical tradition. While traditional Chinese meanings and uses of Chinese you sometimes match with English "there exists/there is," "existence," and "Being," significantly, they often do not. Additionally., in Western philosophical thinking, "existence" and "Being" are often conceived quite differently, sometimes oppositely, and, yet, the same Chinese term you is used for translating both. 
When Chinese you is used in more complex Chinese fabricated terms which translate Western philosophical notions into Chinese, then, the task of translating from Chinese back into English is straightforward. On the other hand, when Chinese you is used alone, it is only very careful discern ing and thinking as much as possible as a Chinese person would that allows one to make accurate judgment as to whether an instance of Chinese you follows traditional Chinese usage or is a Chinese translation for a Western philosophical term - and in the latter case, to make additional accurate judgment as to which Western term is intended. Here, also, we would suggest that a new method of rendering Western philosophical terms in to Chinese, which does not simply substitute Chinese terms for Western ones, is needed. Without such a new method, it becomes very difficult to explore comparatively in what ways Chinese you is similar to English "existence" or "Being" and in what ways it is different. This question is very important to evaluating Feng Youlan's philosophical work as will be seen in the essays of this volume.

Translation of papers of this volume proceeded fairly easily, using customary matching of Chinese you with English "to exist," until the articulate essay of Professor Chen Lai simply forced an entire re-thinking of, and eventual rejection of, this customary matching. After several translation efforts, I found that if I translated Chinese you with English "exist" or "there exists/there is," I could not bring together into one English conversation (as Professor Chen was doing in Chinese) both Feng Youlan's use of you and traditional Chinese philosophy's use of you.

Thus began some rather serious and lengthy exploration of what at root is the problem. Having located at least one source of the problem, solving the problem required more literal and consistent rendering of Chinese you into English as "to have" rather than "to exist," even though Feng Youlan himself states that Chinese "you" is Chinese "cunzai" (exist). ${ }^{13}$ As will be set forth in detail in Part Three of this Introduction, Feng's use of Chinese you is traditional enough in linguistic pattern and meaning to enable a literal translation of you as "to have" or "having" in English for his writings as well as for those of other authors of this 
volume. This one consistent, more literal translation of Chinese you as "to have" or "having" in all essays in this volume, not only has allowed Feng and his critics to talk with one another in English, but also, it has allowed for that conversation to be exacting and make sense.

Adopting this solution, however, meant re-translation of each of the other essays in this volume using the new more literal approach to Chinese you. Initially, a reader of English may find a more literal translation of Chinese you as to "to have" or "having" somewhat less fluid. But our purpose here is not poetry, but rather precise, detailed philosophical discernment and discussion which cannot make the same demands on fluidity of translation as literature does neither can science for that matter.

One hopes that the reader will find, as the Editor has, that a more literal and exact rendering of Chinese you into English offers increased insight into aspects of Chinese thinking not usually open to Western view. In fact, the Editor has found that questions uniquely posed in Chinese metaphysics suddenly appear lucidly in English translation when previous customary or notional substitutions of Western philosophical terms for Chinese philosophical terms tended to obscure more than they revealed. Importantly, use of more literal translation of Chinese you as "to have" or "having" has been far more efficient in that one has needed far less explanatory annotation in Endnotes to each paper.

Solving these translation problems concerning Chinese you and $l i$ is essential to any translation of Feng Youlan's philosophical work and the work of those analyzing Feng Youlan's philosophy. Put simply, while a bridge encouraging initial inter-cultural exploration has already been built by customary substituting of some Western terms for Chinese terms and the other way round, the time appears to have come for a re-examination of these customary matchings or substitutions and for a more exacting method of communicating ideas from one language to another that allows deeper, comparative questions to be asked. If we do not continually seek greater precision in this regard, we shall ever be at risk of discussing Western philosophy while thinking we are discussing Chinese 
philosophy.

In this volume, then, we have tried to enable Chinese thinking to speak literally in English as much as possible. The philosophy and writing of Feng Youlan, who thought and worked in both English and Chinese, demand this. Translations in this volume are the result of many drafts and revisions of drafts. I leave the reader to decide whether or to what extent these efforts at more literal translation of Chinese philosophical thinking succeed.

To address these and other problems of translation in a manner helpful to the reader, the Editor offers the following forms of assistance.

First, specifies of the solution which has worked for this volume in translating Chinese you more literally as "to have" or "having" rather than English "there exists/there is," "existence," or "Being" are detailed in Part Three of this Introduction. Those with strength of heart-mind enough to work through Part Three, having there explored semantic and syntactic constructions of Chinese you (have, having), zai (is [present] in), cunzai (exist, existence) and $w u$ (not have, not having), will find rewarding the last sub-section on translating specific terms of Feng's thinking. Those desiring simply a list of Feng's terms and this volume's translation of them will find such a list in the last sub-section of Part Three.

Second, while working in grammatical English, the Editor has sought to preserve, when possible, Chinese word order in English.

Third, we present both English translation and Chinese pinyin romanization for significant terms under discussion. The usual pattern is to offer English translation first, followed by pinyin romanization in parentheses. However, we invert this order to Chinese followed by English in parentheses or no English at all in three cases:

1. when discussing the four central terms of Feng's New Lixue system: li (pattern, principle), qi (energy-matter), daoti (embodiment of dao [Way]), and daquan (great whole);

2. when our concern or attention is with the Chinese term or expression itself; 
3. when a Chinese term is used repeatedly in discussion such that English translation is no longer necessary.

With this combined approach, it is hoped that the reader of English or Chinese or one beginning study of Chinese will be able to track with precision the matching of certain Chinese terms with English terms.

Fourth, for readers of Chinese, a Glossary of Chinese terms used frequently in this volume is presented at the end of the volume. Romanization of Chinese terms together with Chinese characters appear in alphabetical order.

Fifth, when questions of translation do arise, to clarify an author's intention, the Editor has offered in the text and in the Endnotes some annotated explanation of terms put in brackets [ ].

The author of each paper is responsible for the Endnotes of the paper. In some cases, following Chinese academic custom, Endnotes were submitted in a much abbreviated form and needed considerable amplification for publication in English. To assist with this, the Editor returned to Beijing University to discuss and complete notes with each author. Nonetheless, some notes still remained for the Editor to work out and to provide additional information. Additional material in the translated text and Endnotes that has been provided by the Editor is set off with brackets [ ] to indicate clearly where the Editor is responsible for information so that no misrepresentation of an author is made. In the Endnotes, titles of Feng Youlan's writings appear without mention of Feng Youlan as author.

A Selected Bibliography of works mentioned in the papers and in the Endnotes may be found at the end of the volume. Within this Selected Bibliography, works of Feng Youlan are listed separately according to collection and date of publication. Assistance with dates, titles, publishers and translations of Feng Youlan's writings has come from a Bibliography prepared for the "International Seminar on the Thought of Feng Youlan" by Cai Zhongde, the husband of Zong Pu, Feng Youlan's daughter. He is currently preparing a complete bibliography of 400,000 characters, including all of Feng's published and lesser known writings. 
Compiling, editing and translating for a volume such as this involves many unforeseen tasks. The Editor made two trips back to Beijing during the summers of 1992 and 1993 for these purposes. She wishes to thank Feng Zong Pu whose hospitality and helpfulness on both occasions are gratefully remembered and appreciated. During the most recent visit, the Editor remembers sitting in the Feng living room with Professor Tu Youguang, ${ }^{14}$ General Editor of Feng's Sansongtang Quanji (The Collected Works at the Hall of Three Pines), and Professor Tu's student John Flower, a Ph.D. candidate from the University of Virginia, who has lived and done research in the Chengdu region for several years, as we carefully went over Professor Tu's essay for this volume. One sensed Professor Feng sitting across the table in his usual chair. The work continued on into twilight as Feng's great library of old, traditionally bound books along the walls receded into darkness. As though from far in the distance, the sound of Zong Pu's dot-matrix printer in an adjoining room wafted to our attention. The inevitable new infusing the old.

It was fitting, although altogether unplanned, that on my final day in Beijing this past September, with all tasks completed and most questions, at least for the moment, answered, 1 returned to the Feng family home. Zong $\mathrm{Pu}$ in her gracious manner responded to my last queries and notes and then, as a final blessing, invited us ${ }^{15}$ to the last room, around the inner quadrangle, along the old wooden floors, at the back of the Feng family home. There a family shrine was laid with pictures of Feng and his wife illumined in soft light. Around the room on all sides werc Feng's original writings. High on one of the wood-andglass bookcases were two worn leather suitchases - those which Feng had carried to Columbia University on his first trip from China in 1919? Quietly I was left for a final moment of recollection and silence. Then we were gone through the garden of Three Pines, leaving the gate open.

Part Two: The Essays

Papers of this volume are arrayed into sections according to questions xii 
and issues discussed as well as to significant developmental moments in Feng Youlan's thinking over time. Section One entitled "Perspectives" presents a translation of the documentary film and Mr. Thanh's remembrance of the gaobie (goodbye) ceremonies along with his review of main issues and debates of the conference. Section Two entitled "Questions of Culture and Epistemology (1920s-1930s)" considers Feng Youlan's contributions to Chinese intellectual debate on Chinese and Western culture and pertinent epistemological problems therein. Section Three entitled simply "New Lixue (1940s)" settles into thorough-going presentation, analysis, and assessment of Feng Youlan's deeply and broadly conceived "New Lixue" philosophical system. Section Four entitled "Marxist-Maoist Themes (1950s-1980s)" considers Feng's enduring post-1949 philosophical responses to and conversation with Marxists and Mao Zedong. Section Five entitled "Alternatives (1980s. 1990s and beyond)" presents work of those who, while standing on the shoulders of Feng Youlan and others, take considerable leave from Feng's kind of philosophical effort to engage in philosophy of quite different kinds.

As regards Section Two, it may be of assistance to those new to Chinese philosophy and China's recent history to provide here a brief synopsis of events involved in and beyond May 4,1919, during which renewed debated about Chinese and Western culture took place. Background and context offered here by the Editor should not be construed in any way as representing the views of the three authors whose papers comprise Section Two.

The May Fourth period or movement is named after a May 4, 1919 incident, when students, numbering in thousands from several universities, marched to Tiananmen (Gate of Heavenly Peace) to protest an agreement within the Versailles Peace Treaty ending World War I. According to this agreement, the province of Shandong was to be transferred from German hands to Japanese hands. Although China had contributed to the Allied cause, the warlord Chinese government needed Japanese support for its own survival. Students, feeling a kind of national humiliation in being 
handed from one colonial power to another, marched to Tiananmen to pressure Chinese leaders to reject the transfer, which, in the end, did take place. As events pressed on, some students marched to the residences of President Cao Rulin and Zhang Zongxiang, burning down Cao's house and beating up Zhang. Thus began a many-faceted period of "awakening" bearing the name "May Foruth" which lasted well into three decades and continues to influence events in China today. ${ }^{16}$

The best of Chinese intellectual criticism took part in the May Fourth movement using every genre: literature, history, philosophy, politics, journalism, and more. Views were many with no two voices exactly the same. ${ }^{17}$ At root, it was a movement against foreign imperialism and internal corruption among China's leaders, but within it developed another movement, the "New Culture" movement, which sought a release from decaying remains of traditional Chinese culture, particularly $R u$ (Confucian) culture, and a life-giving transfusion from Western ideas, particularly science and democracy. A new, wakeful awareness of the May Fourth period solidified into valuing the people, valuing the individual, and valuing the nation. ${ }^{18}$

Many intellectuals who had studied traditionally in China and later studied abroad, either in the West (the United States or Western Europe) or in Japan, heralded these aspirations with a view to the effect that China is weak in culture and the West is strong; therefore, China should abandon its own heritage (if not all of it, then most of it) and learn from the West. Within these efforts to comprehend better China's current weakness and the West's current strengths, came the formation of a Chinese Communist Party in 1920-1, ${ }^{19}$ which, over the next three decades, would promote a Marxist ideology, both in theory and practice, that, through brilliant campaign and strategy of its own and through political failures of others, would overthrow the old and install a revolutionary new approach to government in 1949 with the founding of the People's Republic of China. As turbulent political changes from 1919-1949 took place, another more subtle shift was taking place as well: multi-voiced analytic sorting through Chinese traditional culture alongside critical sorting through Western 
culture was reluctantly, perhaps, unintentionally abandoned. In its place single-voiced allegiance to Marxism and, later, Maoism, took both assent and ascent. ${ }^{20}$

In the early 1920s, however, times had been different as hard work in sorting through strengths and weaknesses of Chinese culture and Western culture carried forward earlier efforts of Yan Fu (1853-1921), Kang Youwei (1858-1927), Tan Sitong (1965-1898), Zhang Binglin (1869-1935) and Liu Shipei (1884-1919) among others. ${ }^{21}$ This multivoiced, explorative approach engaged a diversity of views including that of Liang Shuming (1893-1988), a young instructor of Indian philosophy at Beijing University, who, in complete sympathy with students of the May 4 demonstrations, nonetheless, was not ready to abandon Chinese culture and, instead, worked to conserve what, in his view, was the best of China's cumulative heritage. It was Liang Shuming in 1921 who re-focused the culture question or "cultures controversy" in a series of lectures entitled "Eastern and Western Cultures and Their Philosophies" (Dong Xi Wenhua ji Qi Zhexue), which in their published form (Shanghai, 1922), made Liang nationally known as a defender of the Chinese $R u$ (Confucian) tradition. $^{22}$

To these many-voiced discussions and explorations seeking to find and to ponder carefully what Chinese culture has in fullness and what it lacks - complex work, to which elder and younger Chinese scholars have been able to return in the last fifteen years wherein deeper, more enduring answers may yet be found - Feng Youlan, a Ph.D. candidate at Columbia University, added his own developing reflections and essays. It is Feng Youlan's early and maturing views on Chinese and Western culture and the epistemological questions which debates on culture raised that Professors Li Zhonghua, Wang Shouchang, and Tang Yijie outline and assess in their essays.

Professor Li Zhonghua begins with an outline of three stages in the development of Feng's cultural views. Initially, Feng, along with others of the 1920s "new culture" movement, worked from what was known on the surface level of cultural differences to the effect that the West was 
characterized as "outward directed" and China was characterized as "inward directed." Then Feng moved more towards examining what Western and Chinese culture share in common. In the 1930s, in a third phase of his understanding, Feng found difference between Chinese and Western culture to be a difference of medieval and modern times. Refining this view, Feng described the difference between medieval and modern as a difference of "methods of production." (This discernment would link well with Marxist and Maoist views on forces of production, which later carried the day in China). By locating the difference of culture in methods of production, China's way forward into renewed cultural strength was made plain.

Professor Wang Shouchang continues this discussion of Feng Youlan's views on Chinese and Western culture by offering an overview specifically of Feng's adoption of ideas and methods of two major schools of modern Western philosophy: New Realism and the Vienna Circle. New Realism enabled Feng to find a connection between his developing, Western influenced ways of thinking, while studying at Columbia University, and the tradition of Song-Ming Lixue. Then the methods of logical positivism, advocated by the Vienna Circle, moved Feng to re-establish Chinese metaphysics on more logically constructed epistemological ground. Feng did so by following strictly the Vienna Circle's methods of logical analysis. However, to correct what Feng perceived as a one-sidedness of the Western positivist approach, Feng added the Chinese intuitive, experiential approach to his system of thought as well.

Professor Tang Yijie takes this discussion of Chinese and Western culture yet further with his insightful exploration of epistemology in Feng Youlan's work Xin Zhi Yan (A New Treatise on the Methodology of Metaphysics). As no traditional Chinese philosophy had developed its own system of epistemology, Feng's plan in Xin Zhi Yan is quite clearly to survey the history of Western methodological use of epistemology to establish philosophical systems. Then, using analytic methods of New Realism and the Vienna Circle, Feng went on to generate his own new metaphysics in Xin Lixue (New Lixue). Through formal analysis of 
events/things and existence, Feng attained four concepts: li (pattern, principle), qi (energy-matter), daoti (embodiment of dao [Way]), and daquan (great whole). Using these four concepts, Feng set forth four sets of propositions. To this positive method, Feng added what he called the "negative method" of direct experience and recognition. With these combined methods, Feng established an epistemological foundation for his "New Lixue" system and made his contribution towards resolving the problem of epistemological theory in Chinese philosophy. Chinese philosophers contemporary with Feng Youlan (1895-1990)-Xiong Shili (18851968), He Lin (1902-1990), Jin Yuelin (1895-1984), and Zhang Dongsun (1886-1962)-also made contrioutions towards resolving the same problem. With these efforts, Chinese philosophers have addressed questions raised by Western philosophy, while yet advancing strengths already within Chinese philosophy.

Section Three's essays on Feng's New Lixue as a philosophical system are replete with detail as to historical circumstances and purposes for which New Lixue as a metaphysical system was generated. Each essay sets forth the main themes and structure of Feng's New Lixue system followed by careful critique. About the circumstances and purposes of his New Lixue, Feng Youlan himself has also written. In the last chapter of his A Short History of Chinese Philosophy (written in English), Feng recounts that his Zhongguo Zhexue Shi (A History of Chinese Philosophy), completed in 1934, was translated into English with the first volume being published in Peiping (Beijing) three months before the SinoJapanese War began in the summer of $1937 .{ }^{23}$ The purpose of that work, as Feng affirms, was to apply Hanxue (Han study) ${ }^{24}$ scholarly techniques, as well as Western analytic methods, "to clarify the ideas of these philosophers [of the past]" and "to tell us what the words of the philosophers of the past actually meant to these [philosophers] themselves." The purpose of Feng's history of philosophy, then, was not to tell us what he thought the words of the philosophers ought to mean. ${ }^{25}$ Nonetheless, as a philosopher himself, in "a process of development from the old to the new," Feng wanted to push the ideas of the philosophers of the 
past to their "logical conclusions in order to show their validity or absurdity." This work now is "no longer the scholarly one of an historian, but the creative one of a philosopher." Hence, having finished his history, Feng "immediately prepared for new work" just as the Sino-Japanese War broke out. ${ }^{26}$

During the war (1937-1945), Beijing University, Qinghua University and Nankai University formed the Southwest Associated University which was located for four months in Hengshan before moving farther southwest to Kunming in the spring of 1938 . Feng remembers vividly the four months in Hengshan, a time of national crisis, transposition, and also inspiration, when Feng and his close colleagues, Tang Yongtong and Jin Yuelin, each finished significant philosophical work. ${ }^{27}$ Feng's work, Xin Lixue, was the first in a series of six books he would call Zhen Yuan Liu Shu (Purity Descends, Primacy Ascends: Six Books), ${ }^{28}$ all written during the war. The collection presents what is known as Feng's New Lixue metaphysical system of thought, combining New Realism, Vienna Circle logical analysis, and Cheng.Zhu Lixue. This was the creative effort to which he had aspired in the 1930s, the core of his philosophical contributions, and what many consider today as his highest achievement as a contemporary world philosopher.

Elder among Chinese intellectuals today, Professor Zhang Dainian opens this section on Feng's New Lixue with a discerning historical assessment of Feng's contributions over time. Professor Zhang analyses first the meaning of Feng's title for his Zhen Yuan Liu Shu, which when China was coming into victory in the War of Resistance against the Japanese, was a patriotic effort to put attention towards a new beginning. Then, turning to Feng's New Lixue system of thought contained in Zhen Yuan Liu Shu, Professor Zhang lucidly presents: 1) Feng's theory of "two worlds," namely, actuality and truth; 2) Feng's four main concepts inferred from four main propositions, namely, $l i$ (pattern, principle), $q i$ (energy-matter), daoti (embodiment of dao [Way]), and daquan (great whole); and 3) Feng's theory of "four realms," namely, the realms of spontaneity, utility, morality, and transcendence (Heaven and Earth). 
With the main concepts and structure of Feng's New Lixue in hand, Professor Zhang moves on to assess what was and what was not accomplished in Feng's New Lixue. Zhang acknowledges first Feng's fervent love for his country and his hope for China's people to rise again with confidence upon victory in the War of Resistance against Japan. Second, along with others, such as Xiong Shili and Jin Yuelin, Feng Youlan endeavored to find some link between Chinese and Western philosophy. Feng achieved this through linking what Professor Zhang calls the "orthodox" schools of philosophy on both sides: Chinese Cheng-Zhu Lixue and Western Platonic rationalism, both of which in recent times have declined in prominence. Third, after liberation in 1949, Feng Youlan along with others began to explore dialectical materalism and historical materialism. Feng's decision to move in this direction was self-chosen and based in his search for truth.

Having made this decision, in the 1950s Feng Youlan wrote a series of essays criticizing his own New Lixue system of philosophy. One aspect of this self-criticism was an incisive look at the question of whether $l i$ (pattern, principle) is prior to events/things or whether $l i$ is within events/ things. Whereas earlier Feng had expressed his discernment of $l i$ as $a$ priori, in his later years, Feng argued against $a$ priorism and expressed his discernment of $l i$ as $l i$ within events/things. Feng also criticized his theory of spiritual realms stating that the realm of Heaven and Earth is formed "out of thin air." In Professor Zhang Dainian's view both these selfcriticisms are profound in that they show forth Feng's courage and humility in his on-going search for truth. Professor Zhang further commends Feng for his Zhongguo Zhexue Shi Xinbian (New Edition of A History of Chinese Philosophy) which explores the development of Chinese philosophy using Marxist methods. Feng's aspiration was to nourish the future of philosophy through this Marxist re-consideration of Chinese philosophical history. Feng Youlan poured out all he had, endeavoring to enable China's old culture to be a source for China's new culture. Professor Zhang closes with admiration for Feng Youlan's keeping pace with his times. 
Professor Azuma Juji's essay takes us into exacting exploration of the link Feng Youlan discerned between Cheng-Zhu Lixue and Platonic/ New Realism. Professor Azuma presents first the philosophical particulars of Feng's New Lixue and then goes on to investigate carefully its philosophical sources. Feng's early philosophical development began with initial interest in logic and traditional Chinese philosophy. Then Feng moved on to Western philosophies of Henri Bergson, pragmaticism, and New Realism. As an innovative movement at the beginning of the twentieth century, New Realism took a firm stand against idealism and argued for recognition of objective reality that does not depend upon a knower. Further, what we know of reality presented in thought is not considered "consciousness only" but, rather, objective reality itself before our eyes. These two points would prove very important for Feng Youlan's matching of Cheng-Zhu Lixue with New Realism. Feng's doctoral dissertation at Columbia University in 1924 did not yet incorporate Feng's engagement with New Realist views, but his revision of his dissertation upon return to China later that year did. Building upon initial linkages between ChengZhu Lixue and New Realism which Feng discerned in the 1920s, Feng augmented and integrated these linkages into a complete system of New Lixue presented in his Zhen Yuan Liu Shu of the 1940s.

Professor Azuma carefully lays out one-to-one parallels between New Realist concepts and those of Feng Youlan. Turning first to Bertrand Russell's influence on Feng Youlan, Azuma examines in particular Russell's early philosophy and finds a close match between Russell's analysis of universals of category/class (inclusive of things, events, and relations) as a priori and Feng's analysis of $l i$ (pattern, principle). Further, Russell's universals which subsist and events/things which exist match with Feng's $l i$ which hiddenly exist (qiancun) and shi (events/things) which exist (cunzai). Azuma goes on to trace Feng's trying on and rejecting Russell's later 'Neutral Monism," keeping in the end with Russell's earlier view.

Beyond Russell an even stronger influence on Feng Youlan's thinking was that of W. P. Montague, in his The Ways of Knowing, parts 
of which Feng translated into Chinese in the late 1920s. Using passages from both Montague and Feng, Azuma carefully shows that in holding 1) that every particular has a universal, 2) that universals are more basic than particulars, 3) that universals are independent of particulars and prior to particulars, 4) that universals are not only thoughts in the mind, and 5) that universals, in themselves, are not the whole of an event/thing, Montague's and Feng's positions are closely parallel. Even their expressive forms are similar. Azuma, therefore, suggests that when Feng translated Montague's work into Chinese around 1927, the foundational structure of Feng's own New Lixue system took shape. Professor Azuma closes his exploration of Feng's New Lixue with a look at Feng's dismissal of the Vienna Circle's rejection of metaphysics and at Feng's theory of spiritual realms.

Professor Yin Lujun's essay is the first of two, this and the next by Professor Chen Lai, which offer critique of Feng Youlan's New Lixue. Professor Yin again reviews the basic conceptual theory of Feng's New Lixue and examines the influence of Plato on Feng's thinking. Yin finds that Feng's world of $l i$ has all the characteristics of Plato's realm of idea except, importantly, for Feng, universals and particulars are both real, whereas for Plato only universals or ideas are real. Feng's rejection of Plato on this issue led him to New Realism and eventually back to the Chinese $R u$ (Confucian) view of our everyday world as perfectable.

Examining parallels with New Realism, Professor Yin focuses on a difference in Feng's methodological approach. In arguing for the objectivity of knowledge, the objectivity of ideas, New Realists relied upon the Western traditional of Realism which could be traced back to Plato. Feng, by contrast, endeavors to establish the objectivity of $l i$ (pattern, principle) through formal, analytic statements, an approach which New Realism avoided. Professor Yin takes Feng's "something exists," with which Feng begins to construct his metaphysical system, as a logically true analytical statement. ${ }^{29}$ An analytic statement is one in which the predicate is implicitly contained in the subject. Professor Yin traces how, from "something exists," Feng logically deduces the four core propositions and concepts of his New Lixue system and comes to the conclusions that 
the world of $l i$ subsists prior to things, that $l i$ may subsist without the existence of things, and that $l i$ are real, independent of space-and-time.

Professor Yin points out that Feng Youlan is the first Chinese philosopher to re-construct Cheng-Zhu Lixue using Western logical methods. Feng hoped in this way to bring about a new way for Chinese thinking. Professor Yin criticizes Feng not for making this kind of effort, but for the particular way in which he makes this effort. For, in Professor Yin's assessment, Feng's process of analysis involves a form of invalid reasoning. Professor Yin argues this has been avoided in the West since Kant's distinction of analytic and synthetic statements gave positive refutation to Anselm's argument for the existence of God. According to Kant, "being" or "existence" is not a predicate which can be added to the concept of a thing. In an analytic statement the adding of being or existence to a thing merely posits that thing as an object in relation to one's concept of it. In other words, an analytic statement can make no claim on matters of fact. In analytically deducing class or type of thing from "something exists," then from class or type of thing deducing li (pattern, principle) by which a class or type of thing is discerned as that class of thing, and consequently from there deducing the hidden existence or subsistence of that by which a class or type of thing is discerned as that class of thing, Professor Yin argues that Feng makes the same kind of mistake that Anselm did of attaching a predicate "exist" (in this case, "hiddenly exist or subsist") to a concept.

Professor Yin notes that Feng's use of New Realist themes to reconstruct Cheng-Zhu Lixue through rational analysis of $l i$ contrasts with traditional Lixue scholarly efforts to provide empirical evidence for $l i$. In addition, Feng's analysis of Cheng-Zhu Lixue is not only logical but linguistic in that Feng, like Russell, appeals to the linguistic activity of predication for support of his theory of $l i$ as universal. This contrasts with much of traditional Chinese philosophy which does not find truth as a function of language. Finally, Feng varies from traditional Lixue in not emphasizing understanding acquired through personal experience (tiren). 
Professor Chen Lai in his essay further examines and critiques Feng's New Lixue system. Having been a "conversation partner" with Professor Feng for several years, meeting weekly to discuss writing in which Feng Youlan was currently engaged, Professor Chen Lai's analysis of Feng's thinking is penetrating. Turning first to actuality (shiji), Chen Lai discriminates between 1) "event/thing in actuality" as a certain, individual thing and 2) "actuality" as a universal (category/class name) and as a general name referring to the factual world. Turning to truth (zhenji), it is a logical world in which $l i$ subsist (or hiddenly exist) and is different from our everyday world of actual events/things which exist. $L i$ (pattern, principle) is in (zai) truth and events/things are in (zai) actuality. A question then arises: do events/things have $l i$ ? If events/things do have $l i$, then is $l i$ in actuality? If $l i$ is in actuality, how can $l i$ also be in truth?

To seek an answer to this question, Professor Chen Lai makes careful examination of Feng's claim in Xin Lixue that actuality implies truth. In other words, "having a world of actuality" means "must have that upon which the world of actuality relies by means of which [the world of actuality] is so." Professor Chen Lai points out that giving a category/ class name to something - for example, when one says, "this is dog"is a knowledge determination. We can say that a category/class name is in knowledge, but one cannot go on to say that this category/class or universal or $l i$ exists. For this would be to take a logical a priori as an existential a priori. Professor Chen Lai's criticism here is perhaps somewhat parallel to that of Professor Yin Lujun above, although argued quite differently.

Turning to the question of li's "is in" (zai), Professor Chen Lai examines $l i$ as "that by which so" (suo yi ran), which, in traditional Lixue, is discerned as internally within events/things. According to Feng's New Lixue, $l i$ is objective, real, but not internally within shape-and-form in space-and-time, that is, not within events/things. Therefore, one cannot speak spatially or temporally about $l i$ at all. One cannot say that $l i$ is above events/things nor can one say that $l i$ is within events/things. Rather, according to Feng, one can say only that events/things "rely upon" li; one cannot say that events/things "have $l i$." This being the case, what 
happens to traditional Lixue statements to the effect that "within things" has $l i$ or "above things" has $l i$ ? Feng cannot make such statements, for then $l i$ would change to in (zai) actuality instead of in truth.

By contrast, Feng does state that events/things have nature (xing). However, it is not $l i$ in truth that is a thing's nature, but the manifesting in actuality of $l i$ in truth which is a thing's nature. Similarly, the order or sequence of events/things is not $l i$ in truth, but the manifesting in actuality of $l i$ of order or sequence; $l i$ remains in truth. Hence, in contrast to traditional Lixue which discerns $i i$ as original nature within events/things, Feng's New Lixue places $l i$ in truth and not within events/things as original substance or original nature.

Feng's concept of " $q$ " (energy-matter) also differs significantly from traditional Lixue. Feng's $q i$ "is that upon which events/things in actuality rely as a basis" to become events/things in actuality. As such, Feng's $q i$ is neither a thing in actuality nor truth; it is a logical concept, and like all logical concepts is subjective and not objective in Feng's view. But Feng's notion of $q i$ as a logical concept presents problems. First, qi's lacking objectivity puts it out of balance with $l i$, which in Feng's discernment is objective. Second, qi's lacking objectivity makes it difficult to understand what a thing's relying upon $q i$ as a basis means. In Xin Lixue, Feng distinguishes two concepts of $q i$ : $q i$ as factual existence and $q i$ as logical concept. But then what is the relationship between these two concepts of $q i$ ? Perhaps, suggests Chen Lai, Feng would have done better to let go of New Realism and render $l i$ a logical concept like $q i$.

Finally, turning to traditional Lixue's notions of substance and function, Chen Lai states that we cannot criticize New Lixue by saying that it is not the thought of former $R u$ (Confucian) scholars, for neither was Song-Ming Lixue the thought of former, pre-Qin $R u$ scholars. Feng did not endeavor to pattern after Song-Ming scholars, but rather to receive and to carry forward the work they had done, giving new life to it. With regard to substance and function, Feng's Platonic-styled $l i$ in truth, upon which the form of events/things in actuality relies, is not in actuality, is not internally within events/things, and cannot regulate the movement of

xxiv 
events/things. This differs from traditional Lixue which discerns original li internally within events/things, transforming within $q i$ to become $l i$ of $q i$ and guiding the flow of $q i$, that is, guiding the movement of events/ things. Feng's two world theory in this way greatly differs from SongMing discernment of substance and function as one. Thus, the interconnection between Feng's New Lixue and Song-Ming Lixue is missing on this point. Feng, later, with his notions of intension and extension, began to overcome this difficulty. In final analysis, the questions Feng's New Lixue raises are important ones and part of its contribution is its urging us toward more correct and precise answers.

Papers of Section Four concern Feng's writings of the next thirty years and beyond. Comprehension and appreciation of issues and arguments of these papers require some initial charting of transformative events in China during these three decades. Our purpose is simply to provide a general backdrop for what happened to Feng Youlan and his work during this time. Feng Youlan's Xin Zhi Yan (A New Treatise on the Methodology of Metaphysics), the last of his Zhen Yuan Liu Shu, was published in 1946. Feng Youlan was fifty-one years old. Some would assess this collective effort as the peak of Feng's philosophical work, yet Feng's life was just one half begun.

The year 1946 was a year of triumph as the Chinese people claimed victory in the War of Resistance against Japan. But there were yet other more severe winds blowing in China. The question of who would lead China through reconstruction and modernization, whether the Nationalists (Guomingdang) commanded by Chiang Kai-shek or the Communists (Gongchandang) commanded by Mao Zedong, had not yet been decided. Economic losses and political failures on the part of Guomindang forces rendered them vulnerable to loss of foreign support and paved the way to their eventual defeat at the hands of the brilliantly trained, disciplined and strategically astute People's Liberation Army on the Communist side. In late 1948 and early 1949, with the taking of Tianjin and Beijing and the rest of the North China plain (efforts led by Zhu De, Lin Biao, and Deng Xiaoping), Chiang Kai-shek and the Nationalists were forced to the south, 
later to move to Taiwan as the last bastion of their power. With continuing victorious campaigns south and west, uniting China under the Communist flag, Mao Zedong took the helm. On October 1, 1949, at the Gates of Heavenly Peace, Mao proclaimed the beginning of the People's Republic of China. ${ }^{30}$ Now began the massive tasks of recuperation from a century of war, of centralizing power, of re-education, of building a domestic united front led by the working class, including the peasantry and urban professionals, of land-reform-all following principles of Marx, Engels, Lenin, and Stalin.

Since May 4, 1919, under the influence of a generation of New Culture critics, traditional Chinese learning had been increasingly challenged. Additionally, a great number of teaching personnel at the universities and academies as well as those in scientific professions had studied abroad. During the ascendence of Communist leadership in the 1930s and 1940s, these sorts of intellectuals were given labels such as "feudal," "reactionary," "capitalist," and so forth. Their loyalty to the Communist Party was in question. Nonetheless, intellectuals at home and those returning from abroad were encouraged and reassured that Communist China offered a new way forward even for these. ${ }^{31}$ Many intellectuals initially welcomed the new Communist leadership for the unity. financial security, and lack of corruption it promised. ${ }^{32}$

Yet, as has always been the case in China, there was a tension between political leaders and those of the literati (scholarly-intellectual) class, members of which over three thousand years have taken as their personal responsibility speaking out to those in power on behalf of the people. In so doing, literati have risked loss of position, banishment and even death (including family members), in seeking to urge those who lead to put aside selfishness and to return to care and nurturing of the people. $^{33}$ Just as leaders of the early Han, which also worked to unite a massive China, needed the help of $R u$ (Confucians), i.e. those who were literate, so also those who came to power in 1949 needed the help of the learned to modernize China productively and scientifically. What they did not what from the literati was their independence of thought, their plura- 
lism, and their non-materialist aspirations. Thus, in 1950 would begin a series of warm-up, cool-off campaigns which continued for over a quarter of a century until the fall of the Gang of Four in 1977. By means of these compaigns political leaders sought to bring intellectuals under tighter ideological and political control, a control not previously experienced in China except during the unifying fifteen-year rule of the Qin [221-206], which "buried the scholars" and "burned their books." In Communist China, scholars were not offered, as in China's more traditional past, the dignity of their protests being rejected on the part of those who lead ${ }^{34}$ Instead, in this modern era with new means of communication, intellectuals were required to participate in, and were carefully monitored through, thought reform. These efforts began quietly in 1950 and 1951 and came to a crescendo in the Cultural Revolution (1966-1976) during which China's intellectual elite were brought to self-criticism, jail, and sometimes madness. ${ }^{35}$ Given the three-millenia-old calling of scholars to work on behalf of the people for the promotion of a nurturing and kind government, most intellectuals of the People's Republic of China engaged in thought reform campaigns with a true sense of duty during the early period. Such was their responsibility on behalf of a new Chinese people now being born.

We turn now to the question of how events of this massive turnabout in China's political and economic affairs engaged Feng Youlan.

On May 4, 1946, the Southwest Associated University was closed. In August, Feng Youlan returned to the United States, taking a post at the University of Pennsylvania as a one-year Visiting Scholar in Philosophy. There he began work with Derk Bodde to translate his two-volume Zhongguo Zhexue Shi (A History of Chinese Philosophy). In 1947 the English translation by E. R. Hughes of Feng's Xin Yuan Dao (The Spirit of Chinese Philosophy) was published in London. In the same year, Feng Youlan received an Honorary Doctorate from Princeton University. In 1948, Feng Youlan chose to return to China, stopping en route to teach the Spring term at the University of Hawaii. Then, sailing against the tide of many leaving China, Feng returned to Beijing in the summer of 
1948. That fall Feng was selected as a member of the Central Research Academy (Zhongyang Yanjiu Yuan) in Nanjing, where he went to attend a conference meeting. In this same year, Feng's A Short History of Chinese Philosophy (originally written in English) was published in New York. In 1949 in Beijing, Feng participated in land-reform and gave his support to Mao Zedong's leadership. Back at Qinghua University during the same year, Feng briefly assumed duties as Head of the University's Administration Committee. ${ }^{36}$

In 1950 when half-year long courses in revolutionary re-education for intellectuals began, Feng Youlan, along with tens of thousands of other scholars, participated. The purpose of these courses was to awaken intellectuals to their privileged class backgrounds and to the thought of Mao Zedong, Marx, Engels, Lenin, and Stalin. In small groups each individual's personal case was jointly discussed. Scholars prepared self-critical autobiographies, confessing their past errors as well as those of their fathers and grandfathers. ${ }^{37}$ It is not always possible to know completely the inner intentions of such confessions, which no doubt, at least to some extent, were sincere at the time. In 1950, Feng advanced his first selfcriticism against his New Lixue system. ${ }^{38}$ In 1951, Feng went to India for scholarly exchange and was awarded an honorary doctorate at Delhi University. As all Chinese higher education institutions underwent reform, only one Department of Philosophy remained, that of Beijing University, where Feng Youlan was appointed in 1952.

The start of 1953 brought some relaxation in ideological control, but this was quickly reversed in 1954 with the announcement of the First Five-Year Plan requiring conformity in all sectors of the population. The thought reform campaign continued into 1955 with a refutation of $\mathrm{Hu}$ Feng. Author, editor and Communist Party member, Hu had argued for higher literary standards and greater intellectual divergence of view. ${ }^{39}$ Other intellectuals were criticized as well: Hu Shi (1891-1962) for his Western-informed pragmatism and Liang Shuming (1893-1988) for his conservativism. Feng Youlan along with other intellectuals participated in these criticisms.

xxviii 
In 1956, when China began to move away from the Soviet Union's model of Communism to develop more of an indigenous model, some in leadership positions considered knowledgeable skills of intellectuals essential to the success of the first Five-Year Plan. However, there was a rift on this issue among those at the center of power. ${ }^{40}$ Mao, wanting to work towards improved relations with the people and intellectuals, on May 2nd set forth to a closed session of the State Council a policy of "let a hundred flowers bloom and let a hundred schools contend." 41 When the campaign was publicized in late May, intellectuals were encouraged to express their own opinions in the arts and sciences, but not on political issues. Intellectuals could criticize officials, however, for the sake of improving bureaucratic efficiency. ${ }^{42}$ Under this policy, hesitantly at first, renewed intellectual discussion began to take place in the latter half of 1956. Given the above-mentioned rift among Communist Party leaders and reluctance on the part of Party cadres who feared the emergence of intellectual superiority, the Hundred Flowers campaign was not long afterward temporarily suspended in early $1957 .^{43}$

To get the campaign going again, Mao used much personal persuasion. In January, Mao's collected poems were published for the first time. In February, Mao offered his "On the Correct Handling of Contradictions Among the People" speech, in which he responded to the question of repression..$^{44}$ In Mao's view, non-antagonistic contradictions could coexist in Communist society, but these were to be openly discussed and resolved by what he termed "democratic methods" of reasoning and education. Mao trusted that intellectuals had been sufficiently instructed in his new line of thinking and were loyal. Mao now invited their responsible criticism to keep the leadership responsive to and not alienated from the people. ${ }^{45}$ Taking part in the effort, philosophers began to sort through Chinese tradition for those approaches which, through critique and remoulding, could align with Marxist principles. ${ }^{46}$ To this end a series of debates based on Marxist tenets on how properly to inherit tradition began at Beijing University in January $1957 .{ }^{47}$

According to the Marxist approach, ideas and attitudes are generated 
by social conditions and practical concerns within those conditions. Matter, in this view, is primary; sensation, ideas, and consciousness are secondary. While a Marxist would argue that the world can be objectively known, objective truth comes only through practice, only through living and adjusting to material conditions. So, in the Marxist view, abstract ideas do not exist; they are not material substance. However, ideas or thinking cannot be separated from matter. Rather, there is a dialectical relationship between matter, that is, material conditions, and thoughts or ideas in the mind. This materialist view is linked with politics in that just as matter is primary, so also workers, those whose work engages them in the economic modes of production as the forces of history, are primary and progressive. By contrast, those who promote idealism, that ideas are not tied to material conditions and may exist separately from matter, are the exploiters, the oppressors, the reactionary forces of history.

A. A. Zhdanov, a Soviet Party spokesman on cultural matters, had argued forcefully since 1947 that the history of Western European philosophy was the history of the struggle between materialism and idealism. Zhdanov's view held great sway in China and became the line followed by Chinese philosophers in the early 1950s, when China leaned so heavily towards the Soviet Union. ${ }^{48}$ However, by the mid-1950s Chinese scholars, holding that Chinese philosophy was separate and different from Western philosophy in its focus on ethics rather than metaphysics and epistemlogy, began to argue somewhat more favorably for idealism and the positive role it had played in the history of philosophy. He Lin, a colleague of Feng Youlan's during the War, ${ }^{49}$ had been severely criticized in 1953 and 1955 for "suggesting that idealism had some good points." 50 With the Hundred Flowers campaign of 1956 and 1957, other philosophers who would speak more openly on behalf of idealism began to do so. A few months prior to the January 1957 debate at Beijing University, Feng Youlan published an article, entitled "Two Problems Concerning the Study of the History of Chinese Philosophy," 51 which inquired into the meanings of "idealism" and "materialism" and argued that these terms were not absolute. In his view, many traditional Chinese philosophers had 
both idealist and materialist aspects in their thinking. ${ }^{52}$

Feng Youlan presented a second paper at the January 1957 debates at Beijing University entitled "Zhongguo Zhexue Yichan de Jicheng Wenti" ("On the Question of Inheriting the Legacy of Chinese Philosophy"). ${ }^{53}$ In this piece, Feng argued that while his earlier New lixue philosophy had placed too much emphasis on abstract meaning, rather than concrete meaning, philosophical discussion in the mid-1950s was in danger of going the other way, of putting too much emphasis on the concrete, neglecting the abstract, and thereby neglecting what could be inherited from China's traditional philosophical past. Feng took several examples from traditional Chinese to make his point, arguing from the lun $Y u$ (Analects), Meng $Z i$, and Zhuang $Z i$. In Feng's assessment, principles from all these older texts could be abstracted from the texts, leaving behind their concrete content, and applied in any age. ${ }^{54}$ It was this argument by Feng which caused a great stir. In the months and years of ensuing debate, Feng agreed with his critics that traditional philosophy had to be critically analyzed and transformed, before being inherited, but the question was how. In May 1957, a second conference on inheriting the Chinese philosophical legacy was held which brought even more explicit questioning of the importation of a non-Chinese framework to resolve the question of what could be inherited and what could not. ${ }^{55}$

The swelling tide of debate and criticism with regard to certain efforts of the Communist Party, for example, regarding exclusive control on the part of leaders, restricting access to foreign information and arts, campaigns against landlords, and more, had not been expected, at least not by Mao. ${ }^{56}$ In May and early June 1957, universities around the country joined in as did middle schools. "Democracy Wall" at Beijing University was created for posters expressing diverse individual points of view, often critical of those in the lead. As on May 4, 1919, students took to the streets with rallies for better academic administrative policies and greater academic freedom. ${ }^{57}$

The backlash against the Hundred Flowers campaign began in June of 1957 , driven by those in power who had been reluctant towards the 
campaign in the first place. Mao, too, came over to their side, changing his February 1957 speech to the effect that intellectual freedom was to be used only for strengthening socialism. ${ }^{58}$ In July an "Anti-Rightist" campaign was announced against critics of the Party, even though intellectuals had been officially invited to do so. The brief Spring of "one hundred flowers blooming" came to an end with a thought reform campaign more severe than any previous campaign. Virtually all intellectuals were forced to participate in the anti-rightist campaign, whether they had been critical of the Communist Party or not. Labeled "rightist," losing their professional positions, and in many cases their careers for life, more than 300,000 were sent to labor camps or to jail; some would not return. ${ }^{59}$ As the Great Leap Forward (1958-1962) got under way, the gap between central leaders and intellectuals widened rather than narrowed in spite of the hope of Mao in the Spring of 1956. As creativity on the part of the people was extolled, intellectuals went into even greater isolation. ${ }^{60}$

In 1958 intensifying voices from the left, led by Guan Feng, labelled the 1957 debates on cultural inheritance "revisionist," in that scholars had "revised" Marxist philosophy by obscuring the line between idealism and materialism. They had done so by arguing that there had been materialist approaches prior to Marx, and by arguing that some philosophical propositions transcend class. ${ }^{61}$ Guan Feng's main criticisms were directed towards Feng Youlan and his "method of abstract inheritance," in particular, Feng's examples from Zhuang $Z i$ that an ethical code (dao) could be abstract and beyond class. ${ }^{62}$ In 1959, Guan Feng went yet further in criticizing Feng Youlan's views on Zhuang $Z i$. A formidable Zhuang $Z i$ scholar himself as regards the text and its dating, Guan Feng viewed the man Zhuang $\mathrm{Zi}$ (399? B.C. -295? B.C.) as a reactionary and a negativist. Zhuang $\mathrm{Zi}$ in Guan Feng's view represented the despair and defeat of the slave-owning class to which Zhuang $\mathrm{Zi}$ belonged. Similarly, the thinking of Feng Youlan, as a latter-day student of Zhuang Zi, was deemed reactionary and erroneous. ${ }^{63}$

With this challenge of Feng's views began two years (1960-1961)

$\mathrm{xxxii}$ 
of intense, personalized debate on the dating and content of the Zhuang $Z i$ text. These debates included other well-known scholars in addition to Guan Feng and Feng Youlan. ${ }^{64}$ During these 1960-61 discussions on Zhuang $Z i$, Feng was already under considerable attack for his "method on abstract inheritance." Yet, rather than retreat, Feng chose to head straight into more severe attacks by Guan Feng and others who had political power on their side. In an effort to take Marxist principles seriously, Feng argued for highest knowing in the midst of material experience. ${ }^{65}$ Additionally, Feng argued that while Zhuang Zi's thinking was reactionary, perhaps, in that it was used to ridicule the ruling class in the last days of the slave era, still Zhuang Zi's thinking was progressive in that it was used to criticize the new ruling elite of the Han. ${ }^{66}$

As regards Feng Youlan's participation in the "one hundred flowers bloom" campaign and in subsequent academic/political debates on Zhuang $Z i$, we are offered two glimpses in the first two essays of Section Four. Essays of this section focus on Feng's post-1949 philosophical engagement with Marxist themes. The first glimpse, very brief, yet poignantly full of meaning, is offered by Professor Pang Pu, taking us into the moment of "kaifang" (liberation) and Feng Youlan's return to the classroom in 1957 after years of no teaching. Feng's lectures were on selections from Zhuang $Z i$ and Meng $Z i$. As to $Z$ huang $Z i$, Feng elaborated upon the "pivot of the Way," wherein what is human does not extinguish what is Heaven-given. On Meng $Z i$, Feng lectured on the "unwavering heart-mind" and the "flood-like energy," wherein the ten thousand things are complete in one. self. The former was by way of discussing Feng's realm of Heaven and Earth, the latter was by way of discussing Feng's realm of morality, although Feng could no longer use his earlier terminology to speak about these as he had in his Zhen Yuan Liu Shu of the 1940s. Instead, in the spring of 1957 Feng argued for a non-absolute, non-relative arriving at the center wherein to know the truth.

A second glimpse into Feng Youlan's participation in the Hundred Flowers compaign takes us into Feng's "method of abstract inheritance," a centerpiece of the January 1957 symposium at Beijing University, and 
into responses to this method over time. Professor Yang Disheng presents in his essay excerpts from Feng Youlan's January 1957 paper "Zhongguo Zhexue Yichan de Jicheng Wenti" ("On the Question of Inheriting the Legacy of Chinese Philosophy") ${ }^{67}$ mentioned above, in which Feng argues against neglecting the abstract meaning of philosophical propositions altogether while favoring strongly the concrete meaning of these propositions. The controversy which Feng's paper engendered has lasted until today. In 1958.59 Chen Boda and others challenged that Feng, with this method of abstract inheritance, was merely covering up his idealist New Lixue with a disguise, hiding "restore the old" feudal morality. In response Feng Youlan re-phrased his argument, but kept the core approach, that general principles of the past could be inherited while specific instances in which the principle is expressed would fall away. ${ }^{68}$

Professor Yang traces a tendency in the early years of the People's Republic to deal simplisitically with complex and difficult issues. Yang recounts Feng's and Mao's study-meeting together to discuss this issue and Mao's affirming, "we cannot simplify." Yang goes on to point out, however, that despite Mao's remark, for two decades the problem was not handled well. Two nihilistic thought reform campaigns and years of catastrophe were the result of simplication in handling the question of how to inherit culture. Thirty years later, lacking a resolution to this problem, China still finds itself inheriting the dregs of tradition, whether knowing it or not. Offering examples from military strategy and debates concerning what is right and what is profitable, Professor Yang argues that inheriting the general and not the specific has been going on for thousands of years. The question is how to do this in a Marxist, reasoned manner in contemporary China's socialist age.

Professor Yang goes on to discuss Feng's efforts "to elucidate the old country to assist the new mandate, ${ }^{169}$ a phrase epitomizing Feng's "method of abstract inheriting." Yang discerns that fundamentally the problem of inheriting the past is the problem of the relationship of universals to particulars, a problem discussed in philosophy in every culture, past and present. Feng's conclusion as expressed in his 1984 
Sansongtang Zixu (Autobiography at the Hall of Three Pines) was that $l i$ (pattern, principle) is within shi (events). ${ }^{70}$ By this time, Feng's views had developed over time through incorporating Marxist and Maoist thinking. Feng was ever seeking the truth and offering it as best he could to the Chinese people to build a new nation. Feng respected and trusted Mao Zedong even in his later years. His respect and trust for Mao were an expression of Feng's fervent love for his country and people, motivating him to write a Zhongguo Zhexue Shi Xinbian (New Edition of A History of Chinese Philosophy) using Marxist principles to revise his earlier history of Chinese philosophy. Professor Yang concludes that to the end Feng was convinced that, in a manner consistent with Marxist-Leninist principles, Chinese classical philosophy is an important form of nourishment for Chinese Marxist future development.

As the Great Leap Forward (1958-62) brought with it extensive famine and other disasters, the Anti-Rightist campaign receded, bringing a moderate relaxation of political efforts to control scholars, beginning in 1961 and lasting until mid-1962. Led this time by Lui Shaoqi, this new relaxation sought assistance of scholars toward scientific, technical, and economic development. In philosophy, the early 1960s saw a revival of discussion of Kong $\mathrm{Zi}$ (Confucius), his teaching methods (making no distinction as regards class) and his social ethic which puts the social whole before self-interest. Feng Youlan added to this multi-viewed discussion of Kong $\mathrm{Zi}$ with a series of eleven articles "On Kong $\mathrm{Zi}$ " published from 1960-1963. Outstanding of these pieces is "Lun Kong Zi Guanyu 'Ren' de Sixiang" ("Kong $\mathrm{Zi}$ On Thinking About Benevolence"). ${ }^{71}$ In this piece, Feng cites from Marx and Engels concerning a new class replacing a ruling class. The new class offers its ideas in universal form, expressing that this new class is, in fact, opposed to class and represents the whole of society. ${ }^{72}$ Using this citation from Marx, Feng Youlan argued that ren (benevolence), put forward by the feudal class in opposition to the slaveowning class, is of universal value. Similarly, in his Zhongguo Zhexue Shi Xinbian, the first volume of selections being published in 1962, Feng argues for universality of ren and other traditional Chinese concepts. This 
was the next of Feng's efforts in abstract inheriting, in gleaning resources from Chinese philosophy in a manner consistent with Marxist principles to build a new China.

Feng published a second volume of his New Edition in 1964, but the constant turning of political events delayed publication of the remainder of the work for two decades. While scholars continued to debate materi. alist aspects of $\mathrm{Kong} \mathrm{Zi}$, Meng $\mathrm{Zi}$, Lao $\mathrm{Zi}$ and $\mathrm{Zhuang} \mathrm{Zi}$, increasing priority was given, by those in power, to views of $\mathrm{Xun} Z \mathrm{Z}$ and Hanfei $\mathrm{Zi}$, which easily lent themselves to methods of control. The Cultural Revolution (1966-76) sought complete control of intellectuals, designed as it was to break or smash any vestige of the old, of non-conformity, of individual voice. Feng Youlan was seventy-one years old when the Cultural Revolution began. Detained and criticised, forced to undergo further thought reform, Feng and other scholars suffered deeply. Under these pressures, Feng would again discuss Kong $\mathrm{Z} i$, this time in a more critical manner, ${ }^{73}$ in the early 1970s. Of more enduring value, however, were Feng's continuing efforts during these difficult years to penetrate through to the Way (Dao), to refine his system of New Lixue along Marxist lines in his New Edition.

With the death of Mao Zedong in 1976, the fall of the Gang of Four in 1977, and Deng Xiaoping's taking the lead in 1978, Feng and other intellectuals were able gradually to return to and to publish their philosophical work. Seven volumes of Feng's New Edition were completed and published from 1982.1992. ${ }^{74}$ In addition, Feng Youlan was able to tell his own story of his life, thinking, and final integrations in his Sansongtang Zixu (Autobiography at the Hall of Three Pines), published in 1984. In 1985 began continuous publication of Feng's Sansongtang Quanii (The Collected Works at the Hall of Three Pines or The Collected Works of Feng Youlan). ${ }^{75}$

Professor Tu Youguang, Compiler and Editor of Feng Youlan's Collected Works, transcriber of Feng's Autobiography, translator of Feng Youlan's A Short History of Chinese Philosophy into Chinese, and main discussion partner during Feng's final work on his New Edition, knows Feng Youlan's heart-mind and thinking perhaps better than anyone. It

xxxvi 
is to Professor Tu's essay, the last in Section Four, that we turn for the last word on Feng Youlan's New Lixue system and its re-tuning in a Marxist key.

Significantly, Professor Tu gives thorough-going consideration, more than any other paper in this volume, to Feng's "theory of realms" as the core of Feng's New Lixue system. In fathoming the realm of Heaven and Earth, we are brought back full circle to Feng Youlan's perennial theme, namely, "arriving at highest illumination, one tracks in the way perfectly in ordinary living." This theme Feng Youlan kept in the center of his work even to his final days. ${ }^{76}$ Professor Tu then illumines Feng's reconceptualizing of his New Lixue system, as a result of his participation in agrarian land-reform of the early 1950s, with the terms "intension" and "extension." It is with this re-understanding of his New Lixue, using a Marxist perspective, that Feng Youlan carried on with his New Edition. This was surely the culmination of a life-time of careful, discerning effort.

In Part One of his essay, Professor Tu Youguang puts our attention on the lasting intention of Feng Youlan's philosophical work: to raise the realm of human living. In Feng's discernment, this is the function of any and all philosophical work. Feng calls the highest realm of human living the "realm of Heaven and Earth" (Tian Di jingjie). Feng's Xin Yuan Ren (New Treatise on the Nature of Man) discusses the realm of Heaven and Earth at length and is the central work of Feng's New Lixue system. Even in 1950, in his first self-criticism after thought reform campaigns had begun, Feng with courage used his theory of realms of human living to distinguish between different persons' comprehension of labour. Professor Tu goes on to carefully sort out each of Feng's four realms (of nature, of utility, of morality, of Heaven and Earth). Putting our attention on the last of these, the realm of Heaven and Earth, Professor Tu presents four successive stages within this highest realm (knowing, serving, rejoicing in and being one with Heaven). Professor Tu points out that human reason initially draws a line between Heaven and human person, but when the final stage is attained, this boundary is transcended as human reason itself is transcended. Tu then compares this highest stage with similar 
attainments in other traditions and Western philosophy. Explaining clearly and precisely what this stage of being one with Heaven is, Professor Tu emphasizes that one continues to live within the material world in a moral, useful, and natural manner, while yet having this realm of Heaven and Earth.

From his analysis of the centrality of the theory of realms in Feng's New Lixue, Professor Tu goes on to discuss why human beings have the realm of Heaven and Earth. This is because human beings each have li (pattern, principle) of human being (ren) upon which they rely to be human beings, including having the realm of Heaven and Earth. Professor $T u$, then, presents Feng's metaphysical theory of $l i$, which Feng derived from one affirmation concerning actuality, that is, from one statement of fact: some event/thing exists. In using Western logic to advance a new understanding of Lixue, Feng's New Lixue is quite different from old Lixue. Professor Tu lucidly examines a problem area in Feng's logical derivation of $l i$, a problem area which Professors Yin Lujun and Chen Lai, in their individual essays, have examined as well. Tu's argument is that to make the logical inferences that Feng does, one must stipulate certain conditions as premises. However, these conditions as premises are exactly the cunclusions to which Feng's inferences are intended to lead. So the theoretical demonstration is circular. Given the times of 1948 when these problems were being discussed, resolution had to wait.

Feng continued to ruminate over these problems during his participation in agrarian land reform in 1950. Learning through experiences during that time, Feng grasped universals and particulars newly in terms of Hegel's "concrete universal." Although his new thinking was not expressed in writing until 1985 (in Volume Four of his New Edition), it was originally worked out when working in rural areas and thinking over the problem of class. He discerned that each class has its intension or universal and its extension, which is particulars. From extension, one may infer intension, but not the other way around. Feng's starting point now was changed to category or class name which has li. An individual, as a member of a category or class, is an extension of that category or class 
name. $L i$ is the intension of that category or class name. If an individual relies upon $l i$ of that category or class name to the farthest point, one has the realm of Heaven and Earth.

Although the circularity of Feng's argument was now resolved with this new starting point, there is still the problem that, in Feng's view, category/class has $l i$, whereas in Professor Tu's discernment individuals have $l i$. One problem with the view that category/class has $l i$ is that it offers no meaningful way of explaining or encouraging differences in individual initiative. Another problem is that the only existence that category/ class has is the existence on the part of the individuals of that category/ class. Third, knowledge begins with knowing the qualities and relations between individuals. $L i$ of category/class is a generalization derived from knowing qualities and relations of individuals. In Professor Tu's discernment, the realm of Heaven and Earth manifests not the perfection of category/class, but rather the perfection of an individual. Such is the Daoist understanding of the realm of Heaven and Earth.

Professor Tu Youguang's essay firmly plants us on the solid ground of Feng Youlan 's integrative, refined, biographically reflective work of the early 1980s, a freer, more relaxed period for scholars than had been the case since the 1950s. Essays in Section Five of this volume take us one step further into the late 1980s, the early 1990s, and beyond in the work of two contemporary Chinese philosophers. Professor Zhang Liwen and Professor $\mathrm{Li}$ Zehou both offer assessment of the kind of philosophy in which Feng Youlan and others have been engaged. In addition, they argue for new approaches or new alternatives in Chinese philosophy, each connecting with a dimension of Western philosophy different from that with which Feng Youlan connected, i.e. New Realism. Throughout these two essays an important question again and again emerges: on what grounds, by what criteria, within what boundaries, if any, is one's philosophical work deemed tracking the Way (Dao)?

Professor Zhang Liwen's paper opens his discussion of Feng Youlan's work and of an alternative way of doing Chinese philosophy, with an historical outline of three schools of Song-Ming Lixue: 1) that which 
takes $l i$ (pattern, principle) as the root, 2) that which takes xin (heartmind) as the root, and 3) that which takes qi (energy-matter) as the root. Professor Zhang then goes on to explore developments in the 1940s within each of these schools of study (xue), calling these: New Lixue, New Xinxue, New Qixue. All three schools consider their work to connect with and succeed (jiezhe) Song-Ming Lixue. Professor Zhang understands this connection to mean that half of what one is thinking comes from those who have come before, and the other half comes from oneself. Zhang offers Feng Youlan's New Lixue as a good example of such connection in that Feng's truth and actuality carry forward Song-Ming Lixue's "substance" ( $t i)$ and "function" (yong), respectively. Another example of connecting with and succeeding Song-Ming Lixue may be found in what Professor Zhang calls "New Xinxue." He Lin, taking heart-mind as $l i$ and substance, took thing, which manifests heart-mind, as function, and carried forward the teaching of Lu Xiangshan and Wang Yangming. To the thinking of these scholars, He Lin also brought the thinking of Kant, Hegel and Spinoza by way of adding explanatory interpretation. "New Qixue" is exemplified in the work of Zhang Dainian [1909. ], who carries forward the study of qi established by Zhang Zai in the Song period and links it with contemporary Marxist materialist philosophy.

From 1949 onward, New Ru (Confucians) (Xin Ru Jia) of the New Xinxue view in Hong Kong and Taiw an criticized Feng Youlan in his New Lixue for neglecting Cheng-Zhu teachings on heart-mind $(x i n)$ and inborn nature (xing). Moreover, these scholars argued for the primacy of LuWang Xinxue teaching in Song-Ming $R u$ (Confucian) study, with Cheng. Zhu Lixue teachings as a branch of Song-Ming $R u$ (Confucian) study. During these same years, on the Chinese mainland, New Qixue scholars explore the developmental history of Qixue along with its theoretical integration in Marxist thought.

Professor Zhang, however, notes a problem with these efforts in that these "new" schools have now become systematized in themselves and are in need of renewed creativity, of higher level discussion which comes from within oneself. Each of these three new schools has not yet gone

$\mathrm{xl}$ 
beyond the philosophical tradition of metaphysical ontology which investigates the original source - whether $l i, x i n$, or $q i$-of the world as we know it. Science and technology of today urge a new kind of philosophical work. Secondly, these three points of view have had a tendency to become dictatorial and exclusivistic, claiming what they discern to be the source as the one true, good, and beautiful, while all else is false, evil and ugly. When these views are linked with political power, the result can be to use $l i, x i n$, or $q i$ to "kill" people in the absence of objective legal procedures. Thirdly, these three schools lack critical self-consciousness without which they lose their creative transforming vitality.

A new approach to philosophical theory would be what Professor Zhang calls the "study of harmony and integration" (hehexue). Zhang gives a preliminary outline of this new approach, linking major concepts in Chinese philosophy with Marxist dialectics and quantum theory in physics. First, all events/things are produced within a process of moving transformation. Within this movement, a tendency towards equilibrium causes events/things to manifest definite qualities and inter-relationships. Next, opposition and unification of different qualities result in a fusing together which becomes a new event/thing. Finally, what is produced blends characteristics of both, such that the best of each quality shows forth more fully. Using this kind of approach, Zhang urges rejection of mechanistic determinism and greater comprehension of the fact that any form of understanding includes the examiner within the examined and, thus, is relative. The theory of harmony and integration studies events/ things in motion and transcends Song-Ming $R u$ (Confucian) study and its successors, which analyze stillness. Professor Zhang concludes that the theory of harmony and integration best expresses China's cultural essence and vitality.

Professor Li Zehou sets forth yet another approach to doing Chinese philosophy today which differs both from traditional Chinese philosophy and from Zhang Liwen's alternative to traditional Chinese philosophy. Professor Li Zehou, leaning more towards a link between Chinese philosophy and Western continental phenomenology and existentialism, starts 
his philosophical discussion with what his calls the "proto-phenomenon" of "I live" or "one lives" (ren huozhe). This proto-phenomenon or base contains three questions: 1) How do I live?, 2) Why do I live?, and 3) In what manner do I live? Li discerns "I live" as a kind of unchosen "throwness" into an existence shared with others in the world. "How do I live?" comes before "Why do I live?" Answering "How do I live?," which is always together with others, eventuates in the creation of tools, including language. Yet it is answering "Why do I live?" that gives existence its authenticity. Answering this question requires that each person investigate, discover and choose for oneself why one lives.

Answers to "How do I live?" and "Why do I live?", in turn, blend together to answer the third question, "In what manner do I live?" In other words, in what realm of living do I live? Here Professor Li reviews Feng Youlan's theory of realms and Feng's view that philosophy consists in raising the realm of human living. Professor $\mathrm{Li}$ assesses that Feng's theory of realms connects with and succeeds Chinese Song-Ming Lixuc, what Professor Li calls "New Ru study" (Xin Ruxuc). Feng's contribution in this line of connection and succession could be considered part of "modern New Ruxue" (xiandai Xin Ruxue). ${ }^{77}$ Professor Li finds a link between his own aesthetic realm and Feng's realm of Heaven and Earth.

However, the point which Li Zehou wants to make here is that the effort which Feng Youlan made in his New Lixue is one means among others for opening up and reviving the spirit of Chinese tradition. What $\mathrm{Li}$ Zehou is calling for is a "modern Ruxue" (xiandai Ruxue), which is different from Feng Youlan's and others' "modern New Ruxue." What Li Zehou would call "modern Ruxue" is "post-Marxist Ruxue hermeneutics."

Professor Li then turns to those who would object to Feng Youlan's being included in the group called "modern New Ruxue." To some, Feng's political character and his focus on a world of $l i$, rather than upon $l i$ in action, suggest that Feng does not belong to this group. In contrast with these views, Professor Li holds the view that modern New Ruxue in its wider sense includes not only the continuation of the Lu-Wang school, 
which may be considered the main school, but also the Cheng-Zhu school. Because Feng Youlan's New Lixue in fact does connect with and succeed Cheng-Zhu explanations, Feng Youlan in Li Zehou's assessment does belong to modern New Ruxue.

The papers of Section Five present two approaches to doing Chinese philosophy today which are quite different from each other and from Feng Youlan's approach. Their efforts differ from those of Feng Youlan in that they do not seek to connect with and succeed (jiezhe) Song-Ming Lixue. Instead, each sees his approach as breaking from what has come before. Still, both Professor Zhang Liwen and Professor Li Zehou in their own remarks about their philosophical efforts evince an intention to inherit, revive and carry forward the spirit of Chinese culture and tradition. More than this, they seek to carry forward the spirit of Chinese culture and tradition by participating in a larger world philosophy as inheritors and enliveners of Western philosophical discernment as well. In these intentions and efforts, their philosophical work is not unlike Feng Youlan's own.

\section{Part Three: Chinese Philosophical Terms in English Translation ${ }^{78}$}

\section{Guidelines}

Translation and critical discussion of Feng Youlan's philosophical work brings to the surface just about every major issue in Chinese philosophy today as well as many issues in comparative philosophy, none of which we can adequately pursue here. Our purpose, rather, is to cut a path through to a few terms important to Feng Youlan's philosophical thinking and to explore alternative matches for these terms in English translation.

In the main, we attend to discernments, linguistic patterns, and arguments for matching Chinese "you" more literally with English "to have" and "having," rather than matching it notionally with English "there exists," "existence" or "Being." Necessarily involved in a discus- 
sion of Chinese "you" are discussions of Chinese "zai" (is in) and "cunzai" (exist, existence). Additionally, we shall examine Chinese "qianyou" (literally translated as "hiddenly have" or "hidden having") and "qiancun" (literally translated as "hiddenly exists" and "hidden existence"). Feng used qianyou and qiancun to translate Western philosophical terms "subsistence" and "subsist" respectively. In so far as Feng and his critics discuss whether things have (you) li (pattern, principlc) above them (wushang) or whether things have (you) li within them (wuzhong), a discussion of Chinese you also involves some consideration of $l i$ and how $l i$ is discerned. Ways in which Chinese terms may be translated into English using "is" or "to be" will emerge, inevitably taking us to the work of Angus Graham on the topic. Using translated examples, we shall compare some differences between Graham's approach and our own.

In examining alternative translation possibilities, it is abundantly clear that simply by the choice one makes of words in one language to match those in another language, translation is interpretation, no matter how true to an author's intention one seeks and endeavors to be.

Three guidelines direct our translations. First, ways of formulating philosophical questions emerge, in considerable part, from syntactic and semantic contexts. As Angus Graham has described, "the way we think is affected, not only by the language we speak, but by the grammar we impose on it and by the languages in which the problems were originally stated." Further, Graham acknowledges that "each language has its own sources of confusion, some of which are exposed by translation [from one language] into the other. ${ }^{" 80}$ Experience in translating essays of this volume confirms that philosophical discernment and linguistic expression of philosophical discernment are intimately related. Moreover, philosophical discernment and linguistic expression of philosophical discernment differ from one language to another. In this volume, we have tried to hold onto Chinese philosophical discernment and Chinese linguistic expression of that philosophical discernment in English translation. In this manner, we seek to keep open a unique Chinese window on philosophical inquiry even in English translation.

xliv 
Second, no one language, together with philosophical questions expressed through that language, is a better or worse "instrument of thought." ${ }^{81}$ In other words, we cannot take one language's (or a group of languages') ways of speaking and philosophical questions expressed through those ways of speaking as standard for another. Focusing on the Western term "Being," Graham observes that "the verb 'to be' is a characteristic of Indo.European languages which is highly unusual." He further observes that languages outside this group use terms such as "have," "this," "make" in places where English uses "to be" or "Being."82 Graham concludes that "there is no concept of Being which languages are well or ill fitted to express." ${ }^{83}$ "Being" is one way of talking about our world and our engagement in it, but is not the standard way of talking about the world and our engagement in it. Therefore, in this volume, we have chosen not to take the Western-European notion of "Being" as standard for questions of Chinese philosophy and have not matched Chinese "you" with English "Being." Nor have we matched Chinese "wu" (the negative of "you") with English "non-Being." It is our view that these notional matchings are often misleading in that they not only suggest that Western "Being" is standard for Chinese thinking (or any thinking), but also eclipse what is unique in traditional and contemporary Chinese philosophical discernment.

Third, matching conventions, customs, and uses of terms between languages should include matching of syntactic patterns in which terms are imbedded. In other words, between languages we need to find not only a semantic match of terms (those which would appear to refer similarly to events/things in the world), but also a syntactic match of patterns in which these terms relate to other terms within a language. Finding both semantic and syntactic matches of terms yields more literal translation, which, in our view, reveals more of what each language and thinking have to tell us.

Our effort here, as Graham admits about his own elsewhere, is "to pin down a problem which will remain elusive for a long time to come." One hopes with this effort to encourage others restless with problems of 
earlier translations to add their voices as well to clarify this "obstinately obscure subject." 84

\section{Chinese Actualist Discernment}

Our main thesis is that Chinese thinking, interrelated with visual characteristics of Chinese language itself, is "actualist." 85 That is to say, discriminations or patterns of activity on the part of the human heartmind $(x$ in) are deemed to cohere tightly with patterns discerned in actual events/things in the world. To go one step further, in Chinese actualist discernment, patterns of discrimination in the human heart-mind are deemed to be one and the same as patterns discerned in actual events/ things. The device or instrument which conveys this coherence or unity between patterns of discrimination in the heart-mind and patterns discerned in actual events/things is language, in particular, the linguistic function of naming (ming). ${ }^{86}$

A pattern of discrimination on the part of the heart-mind is given a name, a mnemonic device imaged in a character. A name, together with its visual character, points to or refers to an actual event/thing. In so doing, a name, together with its visual character, the latter itself being a pattern of lines, conveys or connects a pattern of discrimination in the heart-mind with pattern discerned in an actual event/thing. ${ }^{87}$ In this manner, a name, together with its visual character, corresponds with a patterned event/thing in the actual world. We need to take account of four other points regarding names.

First, a pattern of discrimination in the heart-mind is deemed to be in one-to-one correspondence, via a name, with a pattern discerned in one actual event/thing. ${ }^{88}$ That is to say, names are specific: one name corresponds to one event/thing. ${ }^{89}$

Second, names compound together. That is to say, while holding to the view that one name corresponds to one event/thing, in everyday use, it is a combination of names which corresponds to one actual event/ thing. If names did not combine to refer to one actual event/thing, the 
list of names would be as long as the number of separate events/things in the world, that is to say, infinite. In fact, however, every name combines with other names such that one compound name refers to one specific event/thing in the world. To illustrate, the Chinese people have almost always not been concerned with "chair" or "chairness" abstractly, but, rather, for example, with "the third chair to the left of the person at the head of the table." Knowing the specific, combined name of that one chair in a specific time and place, one knows how to act vis a vis that one specific chair. It is the appropriate action in this example, sitting or not sitting in the "third chair to the left of the person at the head of the table," depending upon one's relationship with the person at the head of the table and others to his or her left or right, which is what matters in Chinese society and thinking-not universal "chairness," which cannot possibly inform one whether to sit or not to sit in a specific chair. ${ }^{90}$

Third, as just illustrated in the above example, patterns of discrimination in the heart-mind, which are deemed to be in one-to-one coherence-via names (ming)-with patterns discerned in actual events/ things, are not patterns of stillness but patterns of action. ${ }^{91}$ In addition, a name or a combined name, associated with a pattern of discrimination in the heart-mind which is deemed to cohere with a pattern discerned in an actual event/thing, is not descriptive of what an event/thing is; to the contrary, it is prescriptive of what an event/thing ought to do and what someone vis a vis an event/thing ought to do. ${ }^{92}$ Were we to use the Chinese term "dao" (literally, "way" or "path") to substitute for "patterns" or "pathways of activity," we could say that a name conveys or connects dao in the heart-mind with dao in actual events/things. Learning names, then, is to be instructed in dao (pathways of action). Learning names or accumulations of names (oral-written tradition) enlivens dao in heart-mind so that how one lives aligns with dao in actual events/things.

Fourth, names (together with visual characters) that are associated with patterns (pathways of activity) in the heart-mind, which themselves are deemed to cohere with patterns (pathways of activity) discerned in 
actual events/things, are learned through convention, custom, and use. ${ }^{93}$ In Chinese, practiced use of "this" (shi) and "not this" ( $f e i)$ is one instrument, among others, to narrow down the range in our actual experienced world which social convention and custom determine as corresponding to a name and the heart-mind's discriminations associated with that name. ${ }^{94}$ As one learns a language, one is, at the same time, socialized, "customized," into thinking patterns and values of a cultural group. ${ }^{95}$ Names are collectively known and shared, and, given the mnemonic visual peculiarities of Chinese characters which enable unusually tight cohesion among 1) patterns of discriminative activity in heart-mind, 2) patterns in names and 3) patterns of activity in actual events/things, it is easy to see how a change in names leads directly to changed patterns of social interactions within the actual world. New names, conveying newly cognized patterns of discrimination in heart-mind deemed to be in one-to-one correspondence with newly discerned aspects of patterns in the actual world, result in newly desired ways of socially interacting in that world. These new names function in the same way as the old ones did: to bring social conformity as well as conformity between human and natural activity. ${ }^{\%}$

From early on Chinese scholars have been aware that the same name can be associated with different patterns of discrimination on the part of the heart-mind, which patterns are deemed to be in cohesion with pattern in one and the same actual event/thing. This does not contradict our "actualist" thesis presented here, but further confirms it in that persons are aware that pattern in actual events/things can be discerned differently or incompletely by different persons. While pattern in actual events/things may itself change, our collective and individual discernment of pattern in events/things surely changes, deepens, and transforms over time. One could say that the history of Chinese intellectual thinking is a history of efforts to discern or to know pattern in the actual world from diverging points of view. Differing fragments and corners of Chinese actualist discernment have emerged at different times often giving rise to wrangling debates. That history is not yet closed.

xlviii 
Throughout this history the whole point of knowing, of engaging in patterns of discriminative activity in the heart-mind which are deemed to cohere with patterns discerned in actual events/things, has been to encourage and otherwise enable one's or a group's actions to align with patterned action discerned as already present in (zai) the world. Why? The traditional Chinese discernment has been that inborn or Heaven-given pattern (whether called de [inner moral force] or xing [inborn nature] or xin (heart-mind]) in human persons and in actual events/things is good, life-supporting, and harmonious: Heaven-given pattern is the pattern or pathway of activity leading to abundance, the best that life can offer. No wonder efforts to align with pattern (whether called Tian, dao, li [ritual], or $l i$ [principle]) in actual events/things, to adjust to it, to cohere to it, to live and move with it, have engaged the seriousness and sincerety of Chinese people for thousands of years. The effort has been to track in the pathway of life itself.

One problem that Chinese people have struggled with, given this actualist discernment, is how to arrive at complete, and accurate knowing (that is, complete, accurate engaging in patterns of discriminative activity on the part of the heart-mind) of pattern in the actual world. Precise knowing of patterned action discerned as already present in actual events/ things enables precise alignment of one's actions with patterned action in actual events/things. Such precise alignment leads to abundance, life, and all that is good.

Traditional $R u$ (Confucian) and Daoist views are those which seek to align with patterned action discerned as already present in (zai) events/ things in the actual world. Neither $R u$ or Daoists seek to change pattern already present in events/things or to impose pattern upon events/things. ${ }^{97}$ However, $R u$ and Daoists differ in their views of the extent to which pattern discerned as already present in events/things fully or completely manifests of-itself-so (ziran) in events/things. Daoists tend to take that which is of-itself-so (ziran), untouched by human intentions (wu wei), as complete and perfect manifestation of dao in a human person or an event/thing. $R u$ tend to take that which is of-itself-so as not yet fully 
manifesting dao in a person or an event/thing. In $R u$ discernment, what is inborn or "endao-ed" we could call "the grain in the wood." What is needed is cultivation, pruning, cutting away (xiu yang) "to bring the grain out in the wood,"98 to manifest fully pattern which is discerned as already in the wood. In either the $R u$ or Daoist view, discernment of dao begins with discerning doo in actual events/things here in the everyday world and leads to ever finer discernment of dao in itself in the end. Yet, ever finer discernment of dao in itself in the end does not abandon discernment of dao in actual events/things in everyday living. Rather, ever finer discernment of dao leads to ever truer tracking with dao in everyday life. ${ }^{99}$

In closing this sub-section, we would return to the point that pattern discerned as already present in actual events/things, may be incompletely cognized, known, or illumined in one's heart-mind. Incomplete knowing of pattern discerned as already present in the world results in action which is out of alignment with patterned activity in actual events/things. If such incomplete knowing of pattern is shared by a large number of persons, the result is chaos.

Importantly, in the traditional Chinese view, pattern can be manifested perfectly in actual events/things here in the everyday world. In the traditional Chinese view, what gets in the way of complete knowing of, alignment with, and resulting manifestation outwardly of pattern in human activity and in actual events/things has to do with some aspect of the human heart-mind's engagement with "qi" (energy-matter). Aspects of $q i$ which are considered to block or muddy patterned discriminative activity in heart-mind include self-interest, excess emotion, and lack of study-practice (xuexi). In the Chinese case, the solution to the problem of knowing involves purification of $q i$, that is, some form of self-cultivation which eliminates self-interest, excess emotion, and lack of study-practice. This is true for members of both $R u$ and Daoist schools, although the terms, methods, and what is studied differs between them.

Interestingly, the Platonic Realist school has it somewhat the other way around. For Plato, pattern or idea is real and can be known perfectly 
in the mind. By contrast, manifestation or expression of pattern in actual things here in the world is always incomplete and, therefore, actual events/ things are always to some extent unreal. What gets in the way of events/ things completely manifesting pattern or form is events/things' engagement with matter, not the heart-mind's engagement with matter as in the Chinese case. The upshot of this view is that purification of the mind from effects of matter is not required for complete knowing of idea or form. That is to say, Chinese-style self-cultivation is not required for complete knowing of idea. Instead, because idea is discerned to be logical in the Realist view, the more logical one's thinking is, the more idea is known. Logical rigor does not require elimination of self-interest, moderation of emotions, or study-practice of books or methods from oral-written tradition. Therefore, Western logical training is a kind of learning which is quite different from Chinese self-cultivation.

\section{Chinese "you" (have, having) and "zai" (is (present) in)}

Were we to express Chinese actualist discernment in Chinese, we could put it thus: tianxia you dao (under Heaven has dao). Let us examine the three terms of this expression.

First, "tianxia" (under Heaven) refers to "our everyday world" as we know it. Tianxia also refers to "ten thousand (infinite) events/ things" within our everyday world. Hence, we can translate Chinese tianxia as either "under Heaven" or "all under Heaven." As we shall see, both expressions refer to that which may serve semantically as a general location.

Second, "you" is quite similar, both semantically and syntactically, to English "have." One may have (you) a book (shu), have (you) a friend (pengyou), have (you) a life (shenghuo), have (you) a way (dao), and so forth. Chinese syntactic constructions using "you" are similar to English syntactic constructions using "have" in that the "agent" of you may be a person, an animate being, or an inanimate event/thing. In Chinese, when an inanimate event/thing as a location serves as agent of you, it has a loca- 
tional suffix attached to a noun (which refers to an inanimate event/ thing). In English, the Chinese noun with locational suffix may be literally translated into English using a prepositional phrase. For example, in Chinese one may say "ta you yiben shu" (he has a book). Or, may say "ta de shouli you yiben shu" (literally, in his hand has a book). The usual case in English is that a noun alone, not a prepositional phrase, expresses an inanimate event/thing as a location which serves as agent of having: e.g. the table has a book; his hand has a book, the house has a gate. Hence, in the expression we are considering, tianxia you dao, while "under Heaven has dao" is a literal English translation, a more grammatical English translation is "all under Heaven has dao."

Third, as regards, the Chinese term "dao," Chinese tradition has had several terms for "pattern" discerned as already present in (zai) events/ things in the actual world: tian (Heaven), dao (Way or Path), li (pattern, principle), among others. Each highlights a different facet of the Chinese discernment of pattern in actual events/things. In the discussion which follows concerning Chinese "you" (have, having) and "zai" (is in), we shall start by using the term "dao" (pathway of action) and then turn to " $l i$ " (pattern, principle), a term which $R u$ (Confucians) of the Song-Ming period used as did Feng Youlan.

We want now to take note briefly of some general aspects of Chinese linguistic patterns. We cannot explore here, as we have elsewhere, the "ergative" deep structure of Chinese semantics, which takes action undergone by an object as the primary semantic unit. By contrast, Western languages take a subject performing an action, possibly upon an object, as the primary semantic unit. While Chinese semantic relations may also be expressed as subject-action-object relations, we would suggest that imposing Western semantic relationships upon Chinese expressions may result in some distortion of what is originally intended in Chinese. In this regard, we have found "case order," or even better, "role order," to work best in describing Chinese syntax..$^{100}$

In Chinese expressions, focus is on action which an object undergoes. ${ }^{101}$ An agent in an action which an object undergoes may be known, 
but often may not be stressed in the meaning of an expression. Hence, although a known agent of an action which an object undergoes has a syntactic role in an expression, the agent in this syntactic role may be left implicit and not expressed. Again, semantically, what matters is action undergone by an object. Therefore, a meaningful expression in Chinese need only express syntactically an action (in action role) and an object which undergoes that action (in object role). Moreover, once an object which undergoes an action has been presented or is known, expression of the object in syntactic object role can also be dropped, although it retains its syntactic place, even if unexpressed.

Let us consider how these general remarks about Chinese syntax are exemplified in the expression "tianxia you dao" (under Heaven has dao or all under Heaven has dao). You refers to an action serving in syntactic action role. Dao refers to an object, serving in syntactic object role, which undergoes the action you. Tianxia refers to a location in which the action you, which dao undergoes, takes places. In "you" constructions, the location where action takes places serves in syntactic agent role. But, as stated above, the agent of an action is often unstressed and, although known and having a syntactic role to play, may be left implicit. Hence, Chinese "tianxia you dao" (all under Heaven has $d a o$ ), and "you dao" (has dao) have the same meaning, when the location is known but implicit in the second instance. What is central in the meaning of either expression is the action you (in action role) that dao (in object role) undergoes. Tianxia (in syntactic agent role), as the location where the action takes places, is integral to the meaning of the expression, but not stressed. In addition, once we know an object which undergoes an action-in this case, dao-we can drop explicit mention of the object in syntactic object role and simply state the action in syntactic action role-in this case, "you"-which is a thoroughly meaning. ful and syntactically complete utterance in Chinese. ${ }^{102}$

Another Chinese expression "dao zai tianxia" (dao is under Heaven or dao is in all under Heaven) is in mirror-like relationship with the expression "tianxia you dao" (under Heaven has dao or all under Heaven has 
dao). In this new expression, Chinese "zai" refers to an action which dao in syntactic object role undergoes. Let us take a moment to examine Chinese "zai" before analyzing the "zai" construction of this new expression.

Chinese "zai" refers to an action which in English could be expressed by fabricating an English verb "to in" a location. Normally, "in" is a preposition requiring by rules of English syntax the addition of "is" prior to "in" in order to have a proper English verb, "is in," referring to the action of "in." The English term "is" in "is in," which we use to translate Chinese "zai," is what we would call "floppy is" in that it carries no deeper meaning, certainly no existential meaning or existential claim, but simply, given requirements of English syntax, connects the object undergoing an action and the action it undergoes, in this case, the action of "to in" a location. ${ }^{103}$

We could expand upon our English translation of Chinese "zai" with the expression "is present in" a location. We find this expansion helpful in that it makes clear in English that Chinese "zai" refers to an action. In addition, when the location is known but left implicit in an expression using "zai," "zai" is best translated as "is present" rather than simply "is," as the latter expression suggests an existential claim which is not intended in expressions using Chinese "zai." Accordingly, the Chinese expression "shu zai" is best translated as "a book is present" or "a book is here" (adding a general location word). English statements such as "a book is" or "a book exists" are not what is intended by Chinese "shu zai."

Further, English translation of "zai" (is [present] in) may be modified somewhat depending upon the location in which an object is affirmed to be present. For example, if the location is an inanimate event/thing with an attached locational suffix shang (above, on top), then, "zai" in English becomes "is [present] above, is [present] on top" as in "shu zai zhuozishang" (a book is [present] on top of the table). The same is true for other locational suffixes in Chinese such as zhong (within), xia (below), nei (inside), wai (outside), xian (before, in front of), hou (after, behind), and so forth. Accordingly, English translation of Chinese liv 
"zal" (is [present] in) is modified by changing the preposition in English to correspond with a locational suffix in Chinese. Hence, "zai" may be translated into English as "is [present] above," "is [present] within," "is [present] below," and similarly for inside, outside, before, after, and so forth.

Chinese "zai" may also be used in conjunction with other action terms in Chinese, for example, "zhanzai" (stands in, above, within, below, before, after) or "fangzai" (put in, above, within, below, before, after). In combination with another action term, "zai". indicates the action of "in" or "present in" a location in conjunction with another action under. gone by an object in a location.

In general, in this volume, we translate "zai" simply as "is in" (above, below, within, before, after, and so forth). This translation works well whether "zai" stands alone as a single action term in syntactic action role or whether " $z a i$ " is used in conjunction with another action term in syntactic action role. In some instances, for clarification, we will use an expanded English translation "is [present] in (above, below, within, and so forth)" to make clear that Chinese "zai" refers to an action of "presence in" and not to an existential claim.

If we analyze semantically the second expression (dao zai tianxia) in which "zai" appears, "zai" is an action, "dao" is an object/entity which undergoes an action, and "tianxia" (under Heaven or all under Heaven) is a location where an action "zai" takes place. Syntactic roles in this second expression are somewhat different from those in the first expression "tianxia you dao" (under Heaven has dao), which this second expression appears to mirror. In the second expression, "zai" serves in syntactic action role and "dao," which is an object/entity undergoing an action "zai," serves in syntactic agent role. "Tianxia," where an action takes place, serves in syntactic locative or location role and may be preceded by Chinese " $y u$," which marks terms serving in syntactic locative role.

In the "zai" construction, as in the mirror-like "you" construction, the meaning of an expression centers on an action and an object which 
undergoes an action. However, in the "zai" construction, an object which undergoes an action serves in syntactic agent role and is stressed. Not only is an object undergoing an action given greater stress in the role of agent in the "zai" construction, the location where action takes place serves in locative role and is also given greater emphasis than in the "you" construction. In fact, the "zai" construction is often used to make explicit and precise the location where action takes place, while that location is often left implicit or ambiguous in the you" construction.

In summary, the "you" construction and the "zai" construction in mirror-like ways make the same affirmation: an action is undergone by an object in a location. The "you" construction retains underlying ergative semantic structure in that an object which undergoes an action does not syntactically serve as agent in the expression. A location, serving as agent in the action, is unstressed, and, although having a syntactic role, often remains implicit. The "zai" construction, while retaining ergative semantic deep structure, is syntactically much more rigorous in stressing an object which undergoes an action by placing it in syntactic agent role and in stressing a location in which an action takes place by placing it in locative role.

As with the "you" construction, in the "zai" construction if the location is known and not under scrutiny, it may be dropped from explicit mention while retaining its syntactic place. Hence, "dao zai tianxia" and "dao zai" have the same meaning, when the location is known but implicit in the second instance. Furthermore, if the object in question is known and not under scrutiny, it also may be left implicit, leaving simply " $z a i$ " (is present, is here). This expression of a simple "zai," like its counterpart "you," when the location of an action and an object undergoing an action are known but implicit, is a semantically and syntactically complete utterance in Chinese.

What is interesting about "you" and "zai" constructions is that one construction can always be converted into the other. That is to say, an expression which states that a location has (you) an object can always be converted to an expression which states that an object is in (zai) a loca-

lvi 
tion. Four aspects of these mirror-like syntactic patterns draw our attention.

First, as stated above, "zai" expressions do not make existential claims of the sort that an object is or an object exists. Rather, "zai" expressions intend to affirm "presence in" a location on the part of an object. Given the convertability of "zai" expressions to "you" expressions, "you" expressions also do not make existential claims. Rather, "you" expressions intend to affirm that a location "has" an object. One may want to compare certain specific Chinese "you" and "zal" expressions with certain specific Western expressions that use "exist" or "is," but this would be a comparative analysis of specific expressions that are, perhaps, similar in meaning. One could not, however, on the basis of some sinilarity in meaning between certain specific expressions, go on to say that all Chinese "you" expressions are "equivalent" to English "exist" expressions, such that "you" can substitute for "exist" or vice versa.

Second, in "you" constructions the location in which the action of "having" (you) takes place is often left implicit. By converting a "you" construction to a "zai" construction, not only is the location where the action takes place rendered explicit and stressed, the object which undergoes an action in a location is stressed in the syntactic role of agent. Hence, the "zai" construction makes clear what the more vague "you" construction often leaves implicit: the linking of an object with a location. One could say that the linking of an object with a location is the purpose of either "you" or "zai" constructions.

Third, the meaning of "you" (have) and "zai" (is [present] in) is generally vague. These are actions which, as we have just stated, link an object with a location. Using English passive constructions we could say that when an object is "had" (you) in a location, it is "present in" (zai) a location. Neither "you" or "zai" say very much about what kind of linking takes place between an object and a location. In fact, the kind of object and the kind of location will tell us more about their actual linkage than the terms "you" and "zai" do. That is to say, the precise meaning of "you" and "zai" is highly dependent upon 1) the object "had" or "present 
in" and 2) the location where having or presence takes place. Hence, when translating into English expressions using either "you" or "zai," it is important to make clear in English both the object which undergoes an action and the location in which the action takes place, even if these are left implicit in Chinese.

Fourth, given that the precise meaning of "you" and "zai" shifts according to object and location, apart from English "have" for Chinese "you" and "is [present] in" for "zai," it is difficult to arrive at any English terms which can be used consistently to translate "you" and "zai" in all contexts.

In closing, both expressions, "tianxia you dao" and "dao zai tianxia," affirm that dao (pattern, way, path) is "had" (you) or "present in" (zai) in all under Heaven. If we substitute the phrase "actual events/ things in our everyday world" for "all under Heaven," both expressions affirm what we have described in the previous sub-section as Chinese actualist discernment: 1) actual events/things in our everyday world have (you) dao and 2) dao is present in (zai) actual events/things in our everyday world.

\section{4. "You"(have), "Zai" (is [present] in), and "Cunzai" (exist)}

To discuss Chinese actualist discernment further, we shift now to Song-Ming philosophical terms used by $R u$ scholars. We have already articulated Chinese actualist discernment using the expressions "all under Heaven has dao" (tianxia you dao) and "dao is in all under Heaven" (dao zai tianxia). Using the term $l i$ (pattern, principle), ${ }^{104}$ a term central to Song-Ming $R u$ philosophy, we may express Chinese actualist discernment as "all under Heaven has (you) $l i$ " and "li is (present) in (zai) all under Heaven." Further, as we did at the end of the previous sub-section, we may substitute "actual events/things in our everyday world" for "all under Heaven," yielding the expressions "actual events/things in our everyday world have (you) $l i$ " and "li is present in (zai) actual events/ things in our every day world."

Iviii 
In Song-Ming expression of Chinese actualist discernment, " $q i$ " refers to that which manifests $l i$ (pattern, principle) in actual events/ things in shape-and-form (xing) in space-and-time (shijian) in the everyday world. " $Q i$ " is difficult to translate well in English. In some ways $q i$ is like Greek pneuma or Sanskrit prana, both meaning something akin to "breath." What is intended is a name for fundamental "spirituopsycho-physical matter-energy fluid-concrete stuff" by which all events/ things manifest in actual shape-and-form in space-and-time. $Q i$ is a continuum of "concreteness," more or less subtle or gross, which is patterned by $l i$ (pattern, principle). ${ }^{105}$ For the sake of brevity, to present the spectrum of Chinese discernment of $q i$, from subtle energy of the soul to concrete blocks, we have adopted "energy-matter" or, simply, "matter" to translate both Chinese "qi" and the combined term "qizhi."

Put simply, "that which is manifested" is $l i$, and "that which manifests" $l i$ is $q i$. $L i$ which is discerned as present in (zai) actual events/things is called "substance" $(t i)$ in Song-Ming philosophical terms. Furthermore, the manifestation of $l i$ through $q i$ (energy-matter) in shape-and-form in space-and-time, that is, in events/things of the actual world, is called "function" (yong). That is to say, an event (shi) or a thing (wu) is a function, a manifestation-a setting forth through $q i$ in shape-and-form in space-and-time-of the presence of substance $l i$. In brief, then, $l i$ is substance and $l i$ and $q i$ together is function. We discern substance (ii) through function ( $l i$ and $q i$ ). In other words, we discern pattern or principle in actual events/things in the everyday world.

We may use "you" and "zai" linguistic constructions to express discernment of $l i$ as substance present in actual events/things as function in the everyday world. In linguistic semantic analysis, $l i$ as substance is considered an "object" or "entity" which undergoes an action referred to using either "you" (have) or "zai" (is [present] in). Furthermore, in linguistic semantic analysis events/things as function may serve as a location or place in shape-and-form in space-and-time in which an action, either "you" or "zai," takes place. We have already observed in the previous sub-section that in "you" syntactic constructions, 
a location serves in syntactic agent role as that which "you" (has) an object/entity. In "zai" syntactic constructions, a location serves in syntactic locative role and an object/entity which undergoes an action serves in syntactic agent role and is that which "zai" (is [present] in) a location. In this manner, in both "you" and "zai" statements, events/ things as function serve linguistically as a location. What is stated is that either events/things as function have (you) $l i$ as substance or $l i$ as substance is present (zai) in events/things as function.

Expressions such as "under Heaven" (tianxia), "all under Heaven" (tianxia), "the world" (shijic), "the ten thousand things" (wan wu). "actual events/things in the everyday world," and so forth, may all be used interchangeably with "events/things." Each of these expressions refers to what Song-Ming $R u$ philosophers call function. In "you" and "zai" syntactic constructions, each of these expressions (referring to events/ things as function) may serve as a location in which $l i$ (as substance), serving as object/entity, undergoes an action, either "you" (have) or "zai" (is [present] in).

"You" and "zai" syntactic constructions may be used to express not only discerned linkage between substance and function, but also discerned linkage between one function and another function. That is to say, "you" and "zai" constructions may be used to express discerned linkage between one actual event/thing and another actual event/thing. Linguistically, one actual event/thing serves as object/entity, in syntactic object role, which undergoes an action. Another actual event/thing serves as a location in which action takes place. This location takes syntactic agent role in a "you" construction or syntactic locative role in a "zai" construction. In this manner, for example, "the third man on the left has (you) a very large, blue book in his hand" (zuobian de di san wei de shouli you yige hen da de lan yansi de shu) mirrors "a very large, blue book is in (zai) the hand of the third man on the left" (yige hen da de lan yansi de shu zai zuobian de di san wei de shouli). Neither of these expressions using "you" or "zai" to express discerned linkage between one event/thing (function) and another event/thing (function) is an existential

$\mathrm{lx}$ 
claim. Similarly, a statement expressing discerned linkage between li or dao (substance) with events/things (function) is also not an existential claim. In our analysis, none of these traditional and customary "you" or "zai" expressions makes an existential claim. Rather, "you" and "zai" syntactic constructions are used to express discerned relationship, either between substance and function or function and function.

In our translations of Chinesc "you" and "zai" to this point, we have not used the Western term "exist." We would argue that the issue of existence, as Western philosophers have understood it, has not been central to traditional Chinese philosophy. When Chinese philosophers list and discuss terms and concepts of traditional Chinese philosophy, "cunzai," which is a combined Chinese expression used to translate the Western notion of "existence" into Chinese, ${ }^{106}$ is not usually mentioned.

In the Chinese expression "cunzai," the term "cun" is glossed both as "you" (have) and "zai" (is [present] in). ${ }^{107}$ However, as a combined term, "cunzai," which is used to translate Western "exist" into Chinese, means something quite different from either "you" or "zai" in traditional and customary Chinese usage. Importantly, unlike "you" or "zai," Chinese "cunzai" does make existential claims in Chinese. Thus, we may state that "a book exists" (shu cunzai) or "he exists" (ta cunzai). But these statements are very different statements from the sort we have been considering above such as " $l i$ is in events/things of the actual world" (li zai shiwuzhong) or "a book is in his hands" (shu zai ta de shouli). These latter statements link an object/entity with a location as a way of expressing a discerned relationship. In contrast, "cunzai" (exist) statements do not express a discerned relationship; rather, they express discernment of an event/thing in and of itself.

Strictly speaking, Chinese "cunzai" used to translate Western "exist," applies only to events/things as function, that is, to manifestations of $l i$ (substance) through $q i$ in shape-and-form in space-and-time. Hence, we may state that actual events/things in our everyday world "exist." Furthermore, strictly speaking, we may not state that pattern (whether $l i$ or $d a o$ ) which is discerned in actual events/things "exists." 
In Song-Ming $R u$ actualist discernment, events/things as function are discerned to manifest $l i$ as substance. Hence, in so far as events/things manifest $l i$, that is, in so far as, events/things are the durable extension in shape-and-form in space-and-time of $l i$, then the existence of events/ things gives or lends existence to $l i$ by extension. $L i$ in and of itself does not "exist."

Because the term "cunzai" (exist) ends with "zai," "cunzai" as an expression shares some syntactic patterns with "zai." For example, the statement "a book exists under Heaven" (shu cunzai yu tianxia) syntactically parallels the statement "a book is under the table" (shu zai yu zhuozixia). In so far as "cunzai" (exist) applies only to events/things in our everyday world, the general location "tianxia" (under Heaven or our everyday world) is often dropped from explicit expression. In brief, " $x$ exists" (x cunzai) often substitutes for "x exists under Heaven" ( $x$ cunzai yu tianxia).

Further, in a manner similar to Chinese "zai" expressions, which traditionally and customarily convert to "you" expressions, Chinese "cunzai" expressions may be converted to one recently fabricated type of "you" expression which has developed to accomodate the relatively new Chinese term cunzai which translates Western "exist." Using this recently adapted type of "you" expression, "x exists under Heaven" ( $x$ cunzai $y u$ tianxia) may be converted to "under Heaven has the existence of $\mathrm{x}$ " (tianxia you $x$ de cunzai). We have just noted that in expressions using "cunzai," usually the general location "under Heaven" is known but left implicit. Similarly, in the one kind of adapted "you" construction to which a "cunzai" expression may be converted, "under Heaven" may remain implicit. In this manner, "has existence of $\mathrm{x}$ " (you $x$ de cunzai) is an abbreviation of "under Heaven has existence of $\mathrm{x}$ " (tianxia you $x$ de cunzai). Therefore, just as " $\mathrm{x}$ exists under Heaven" ( $x$ cunzai yu tianxia) converts to "under Heaven has existence of $\mathrm{x}$ " (tianxia you $x$ de cunzai), so also the abbreviated expression " $x$ exists" ( $x$ cunzai) converts to the abbreviated expression "has existence of $x$ " (you $x$ de cunzai).

One further abbreviation can take place with "has existence of $x$ " (you $x$ de cunzai). Under one set of conditions-namely, 1 ) if a general lxii 
location which has $\mathrm{x}$ is "tianxia" (under Heaven or our everyday world) and 2) if $\mathrm{x}$ is an event/thing in shape-and-form in space-and-time-an even more abbreviated expression "has $\mathrm{x}$ " (you $x$ ) may substitute for "has existence of $\mathrm{x}$ " (you $x$ de cunzai). In addition, just as abbreviated " $x$ exists" ( $x$ cunzai) may be converted to abbreviated "has existence of $x$ " (you $x$ de cunzai), so also "x exists" ( $x$ cunzai) may be converted to this even more abbreviated expression "has $x$ " (you $x)$. Significantly, under these conditions (and only under these conditions) both abbreviated expressions " $x$ exists" ( $x$ cunzai) and "has $\mathrm{x}$ " (you $x$ ) make existential claims in Chinese.

However, here we come upon an ambiguity with regard to the abbreviated Chinese expression "you $x$." In the first instance, "you $x$ " is an abbreviation for the longer expression "tianxia you $x$ " (under Heaven has $\mathrm{x}$ ). "You" statements of this traditional and customary sort link an object/entity " $x$ " with a general location "under Heaven" or "all under Heaven." "You" statements of this traditional sort convert to "zai" statements of the form " $x$ zai yu tianxia" ( $\mathrm{x}$ is [present] under Heaven or $x$ is [present] in all under Heaven), which also link an object/entity " $x$ " which a general location. As we have detailed above, these traditional and customary "you" and "zai" statements give linguistic expression to discernment of relationship by linking an object with a location and do not make existential claims.

In the second instance, under a specific set of two conditions (see above), "you x" may be an abbreviation for the longer expression "tianxia you $x$ de cunzai" (under Heaven has the existence of $\mathrm{x}$ ). This one adapted kind of "you" statement converts to a "cunzai" statement of the form "x cunzai yu tianxia" (x exists under Heaven). "You x" (has $\mathrm{x}$ ), as an abbreviation of "tianxia you $x$ de cunzai" (under Heaven has existence of $\mathrm{x}$ ), converts to an abbreviated expression " $\mathrm{x}$ cunzai" ( $\mathrm{x}$ exists). These adapted "you" expressions (in longer or abbreviated forms) which convert to "cunzai" expressions, do make existential claims.

Thus, in the first instance, "you $\mathrm{x}$ " (has $\mathrm{x}$ ), which traditionally converts to " $\mathrm{x} z a i$ " ( $\mathrm{x}$ is [present] in), is not an existential claim. However, in the second instance, when the above stated two conditions pertain, 
that is, when "you x" (has $\mathrm{x}$ ) is an abbreviation for "tianxia you $x$ de cunzai" (under Heaven has the existence of $\mathrm{x}$ ), then "you $\mathrm{x}$," which converts to "x cunzai" ( $\mathrm{x}$ exists), is an existential claim.

We noted above that once an object/entity " $x$ " which undergoes an action is known, it may remain implicit as a location does, leaving "you" alone as a complete utterance. In recent Chinese philosophy, "you," as a complete utterance, retains the same ambiguity as "you $\mathrm{x}$ " does, namely, "you," like "you x," may be an abbreviation for a statement that affirms 1) a location has $x$ (and may be converted to a "zai" statement) or it may be may be an abbreviation for a statement which affirms 2) a location has the existence of $x$ (and may be converted to a "cunzai" statement). The latter is an existential claim whereas the former is not. Hence, "you" alone is highly ambiguous. However, when we make explicit what is implicit and understood with regard to a location and an object/entity which undergoes an action in that location, the ambiguity of both "you" and "you x" statements is largely eliminated.

This ambiguity with regard to "you" and "you x" statements which abbreviate two quite different types of statements is a fairly recent phenomenon in Chinese philosophical discussion. As we have stated above, existential claims have not been central to traditional Chinese philosophial thinking. However, with increasing assimilation of Western philosophical concerns, existential claims and their analysis have become quite preva. lent in contemporary Chinese philosophical discussion today. It is import. tant, therefore, to take special note of this ambiguity with regard to "you" and "you x" statements which can lead to significant confusion. For example, this ambiguity in recent times with regard to the abbreviated statements "you" and "you x" has suggested to some thinkers that all "you" expressions may be converted to "cunzai" expressions, that is, that all "you" expressions are existential claims. But this is the case only when the above two conditions apply, that is, when "you" or "you $\mathrm{x}$ " is an abbreviation for the longer expression "tianxia you $x$ de cunzai" (under Heaven has existence of $x$ ).

To say that all "you" statements are "cunzai" statements moves in Ixiv 
the direction of bringing even more Western philosophical focus on existence into Chinese philosophical discussion. As we shall learn shortly, Feng Youlan's New Lixue does just this. Feng Youlan's efforts in this direction were intentional, to see how far this kind of focus on existence could go in exploring Chinese Lixue philosophy. Our own view is that whilc some "you" statements may convert to "cunzai" (exist) statements, it is important to recognize that in traditional Chinese philosophy, "lou" statements generally convert to "zai" (is [present] in) statements. "You" and "zai" statements give expression to discernment of relationship, whether substance to function or function to function, which is subtly and importantly different from discernment of existence.

\section{5. "You" (have, having) and "Wu" (not have, not having)}

Having examined in some detail how mirror-like syntactic constructions 1) use "you" and "zai" to link an object/entity and a location, and 2) differ semantically and syntactically from "cunzai" expressions which make existential claims, our purpose in this section is to explore different linguistic contexts in which the terms "you" (have, having) and its negation "wu" (not have, not having) occur. By taking account of which object/entity is linked with which location, one or the other of which may be implicit in actual expression, we argue that other than "have" and "not have" (for you and wu respectively) no other English terms can be used consistently to translate these Chinese terms in all contexts. With literal, explicit translations of you and wu in different contexts, one may compare specific instances of you and $w u$ in specific contexts with certain Western philosuphical expressions such as "existence" and "nonexistence," "being" and "non-being," "something" and "nothing." However, in making these comparisons, one needs to remember that these are comparisons and not translations.

At the end of Sub-section Three above, we noted that the meaning of "you" (has) and of 'zai" (is [present] in) is generally vague. As terms which link an object/entity with a location, neither "you" or "zai" offers 
Jetails as regards to how an object/entity and a location are linked. Rather, it is the object/entity and the location linked that tell us more about a discerned relationship than the terms "you" or "zai" do. This is why it is important, when translating into English expressions using either "you" or "zai," to express explicitly in English both the location that has (you) an object/entity, that is, the location in which an object/entity is present (zai), as well as the object/ entity had or present in that location, even if location or object/entity is left implicit in Chinese.

Before setting forth examples of English translations of Chinese "you" and "wu" in different contexts, three further aspects of "you," "zai," and "cunzai" expressions need our attention.

First, how is each of these terms negated? The negative of Chinese "you" in vernacular Chinese is "mei you" (not have) and in classical Chinese is "wu" (not have). "Mei you" and "wu" function syntactically in the same manner as "you." For the remainder of this sub-section, we shall focus on classical "wu." The negative of "zai" is "bu zai" (not [present] in), which functions syntactically in the same manner as "zai." Just as traditional and customary expressions using "you" convert to expressions using "zai," so also expressions using " $w u$ " convert to expressions using "bu zai." Thus, "on the table does not have a book" (zhuozishang wu shu) converts to "the book is not on the table" (shu bu zai zhuozishang).

The negative of "cunzai" (exist) is "bu cunzai" (not exist). We have have noted above that the location in "cunzai" expressions is "under Heaven" (tianxia) and is most often left implicit while yet remaining understood. Furthermore, strictly speaking, "cunzai" applies only to events/things in shape-and-form in space-and-time. With location implicit, an abbreviated "cunzai" expression of the form " $x$ exists" ( $x$ cunzai) converts to one adapted type of "you" expression, namely, "has the existence of $x^{\prime \prime}$ (you $x$ de cunzai), which also leaves the known location, under Heaven, implicit. Similarly, the negative expression " $x$ does not exist" ( $\mathrm{x}$ bu cunzai) converts to one adapted type of "wu" expression, namely, "does not have the existence of $\mathrm{x}$ " (wu x de cunzai).

lxvi 
We have detailed how the abbreviated expression "you $x$ " is ambiguous in that it may be an abbreviated expression for 1) a location has $x$ or 2) a location has the existence of $x$. The first of these two possibilities converts to a "zai" (is [present] in) expression and the second of these possibilities converts to a "cunzai" (exist) expression. The negative expression " $u \cdot u$ " is also ambiguous. " $W u x$ " may mean 1) a location does not have $x$ or 2) a location does not have the existence of $x$. The lirst of these possibilitics converts to a " $b u z a i$ " (is not [present] in) expression and the second of these possibilities converts to a "bu cunzai" (does not exist) expression. In addition, once an object/entity $x$ which undergoes an action is known and remains implicit along with a location, then "wu" alone (like "you" alone) stands as a complete utterance, yet retains the same ambiguity as "wu $x$ " expressions do.

Second, how are "you," "zai," and "cunzai" (also their negatives) nominalized? Generally speaking, each of these terms may serve both as a verb and as a corresponding noun. What we have called "case" or "role" order syntax illumines how Chinese terms may serve in syntactic action role as a verb or in syntactic object, agent, or locative role as a noun. Having made this general observation, in actual practice "you" and "wu" often serve as nouns, "having" and "not having," respectively, while "zai" and "bu zai" do not. "Cunzai" also serves often as a noun "existence" but "bu cunzai" does not serve often as a noun meaning "non-existence."

Third, as we have observed previously, in "you" and "zai" syntactic constructions, both dao or $l i$ and actual events/things may serve as an object/entity linked with a location. ${ }^{108}$ On the other hand, in Chinese "cunzai" (exist) constructions, strictly speaking, only actual events/things in the everyday world may serve as an object/entity affirmed to exist.

Turning now to examples of "you" and " $w u$ " in different contexts, in Chinese discernment the greatest possible location is "under Heaven" (tianxia). We could say that "under Heaven" is the location of all "you" (having). We could even say that "under Heaven" is all "you" (having). With actual events/things in the everyday world, layers of "you" 
(having) are possible. Hence, all under Heaven can have a certain person; that certain person can have a certain book; that certain book can have a certain map; that map can have certain gradings of color, and so forth. In each of these layers of "you," an object/entity (serving in syntactic object role) which is had (you) in one layer becomes a location (serving in syntactic agent role) which has (you) another object/entity in a second layer of "you," and so forth.

However, when a layer of "you" is negative, that is, "wu," then layers of "you" come to an end. For if an object/entity is not had, it cannot, in turn, serve as a location which has anything else in another layer. Hence, when dao is an object/entity which under Heaven has (you), only two layers of "you" are possible. While under Heaven is discerned to have $(y o u)$ dao, dao of itself is discerned as not having ( $w u$ ) anything else. ${ }^{109}$

In Chinese, we may express both these discernments, i.e. under Heaven has dao and dao does not have [any object/entity], in this combined expression: tianxia you dao er dao wu. ${ }^{110}$ The first part of this combined expression, i.e. under Heaven has dao, which expresses Chinese actualist discernment, is so integral to Chinese everyday awareness, that it is often left implicit, but not forgotten. Thus, "dao wu" may stand as an abbreviated expression for the whole combined expression. However, when one encounters an abbreviated expression such as "dao wu" in a text, its meaning remains enigmatic until careful linguistic analysis renders explicit all that is implicit and abbreviated. Let us specify exactly how this may be done.

For any object/entity " $\mathrm{x}$ " taken as a location serving in syntactic agent role in a "you" or " $w u$ " linguistic expression, we may assume an initial discernment expressed linguistically as "under Heaven has object/ entity $\mathrm{x}$ " (tianxia you $x$ ). Once an object/entity " $\mathrm{x}$ " is had (you) under Heaven, it may be taken as a location, serving in syntactic agent role, of a second "you" or "wu" statement wherein a second object/entity is said to be had or not had in that location. Hence, for the expression "dao wu," we may assume an initial discernment linguistically expressed 
as "under Heaven has dao" (tianxia you dao). Once dao is had under Heaven, dao may be taken as a location serving in syntactic agent role of a second "you," in this case, "wu" expression.

In the second part of the combined expression in which dao now is taken as a location serving in syntactic agent role, the action which is undergone by an object/entity in syntactic object role is " $w u$ " (not have). However, the object/entity which undergoes the action of "not have" is left implicit. That an object/entity is left implicit is made clear by converting the "wu" (or "you") construction to a corresponding " $b u$ zai" (or "zai") construction (see above Sub-section Three). "Zai" construction requires that an object/entity serve in syntactic agent role. If an object/entity is left implicit in a "you" or " $w \boldsymbol{W}$ " construction, we must make this object/entity explicit to achieve a linguistically complete "zai" or "bu zai" construction. In seeking to accord with the intention of the expression "dao wu," we take any actual event/thing as the object/ entity serving in syntactic agent role in the "bu zai" construction and in syntactic object role in the corresponding " $w u$ " construction. We make "any object/entity $x$ " explicit by supplying it in brackets. Thus, we have "[any object/entity $\mathrm{x}$ ] is not present in (bu zai) dao" which converts back to "dao does not have (wu) [any object/entity $\mathrm{x}$ ] ." In this manner, we become clear that the expression "dao wu" (dao does not have) abbreviates "dao wu $x$ (dao does not have $\mathrm{x}$ )."

Putting linguistic expression of an initially assumed discernment that "under Heaven has dao" together with a second discernment linguistically expressed as "dao does not have [any object/entity $\mathrm{x}$ ]," we get the combined expression: "under Heaven has dao and dao does not have [any object/entity $\mathrm{x}$ ]" (tianxia you dao er dao wu). We now have two layers of "you" construction, the second layer is a negative "you" construction, that is, a " $w u$ " construction. In this manner, we arrive at full explicit expression of what is implicit in the enigmatic "dao wu" expression.

When we analyze the meaning of "dao $w u$ " as an abbreviation for "tianxia you dao er dao wu," we grasp that dao is discerned as had or 
present in the world, but dao as a location does not have $(w u)$ any object/ entity. In effect, we are stating that although dao is had or is present in the world, dao may not be considered as a location, for it does not have any object/entity $x$ linked with it. In other words, in Chinese actualist discernment under Heaven has dao, but dao cannot be located anywhere in space-and-time; only events/things can be located in space-and-time and may serve as a location with which another object/entity may be linked. We may re-state this point using Song-Ming $R u$ terms, when we say under Heaven has $l i$ as substance, but $l i$ cannot be located anywhere in spaceand-time; only function can be located in space-and-time. This point will be important to discussion of Feng Youlan's philosophy in the essays of Section Three of this volume.

With this linguistic analysis, we see that "you" and "wu" as abbreviated expressions express discernment of "[under Heaven] has [any object/ entity $\mathrm{x}$ ]" or "[under Heaven] does not have [any object/entity $\mathrm{x}$ ]," respectively. If we nominalize "you" or "wu," then literal English translations of "you" and "wu" would be "[under Heaven's] having lany object/entity $\mathrm{x}$ ]" and "[under Heaven's] not having [any object/entity $\mathrm{x}]$," respectively.

For the abbreviated expression "dao wu," we could make one additional analysis of its linguistic structure. In this second analysis, we may take both dao and wu each as an object/entity. " $W u$ " taken as a nominalized object/entity would refer to "[under Heaven's] not having [any object/entity $\mathrm{x}$ ]." We may assume an initial discernment regarding each object/entity dao and $w u$. These initial discernments could be linguistically expressed as "under Heaven has dao" (tianxia you dao) and "under Heaven has [under Heaven's] not having [any object/entity $x$ ] (tianxia you wu)." Once dao and wu are discerned as had under Heaven, we can go on to say something more about each. In this second analysis of the expression "dao wu," we take as its intention to assert sameness with regard to dao and wu. To make this assertion in English, we supply in brackets the English copula "is" between the two terms. In this manner, "dao wu" becomes "dao [is] wu." If we offer complete literal transla-

$\operatorname{lxx}$ 
tion of the whole of this abbreviated expression, including assumed initial discernments regarding dao and $w u$, we have: 1) under Heaven has dao, 2) under Heaven has [under Heaven's] not having (wu) [any object/ entity $\mathrm{x}$ ], and 3) dao [is] [under Heaven's] not having [any object/ entity $\mathrm{x}$ ]. Importantly, this second analysis of "dao $w u$ " draws upon our first literal translation of " $w u$ " as "[under Heaven] does not have [any object/entity $\mathrm{x}$ ]."

By making explicit what is left implicit in abbreviated expressions such as "doo $w u$ " which we have just considered, straight forward literal translation and understanding of an enigmatic expression can be achieved. On the other hand, if we notionally substitute Western philosophical terms such as "non-Being," or "non-existence," or "nothing, void," for Chinese " $w u$ " regardless of syntactic structures which interrelate terms, then, understanding of an abbreviated expression such as "dao $w u$ " becomes confused. For example, if we substitute English "non-Being" for $w u$, then "Iao wu" in English becomes "dao [is] non-Being." This substitution, then, turns the affirmation that "1) under Heaven has dao, 2) under Heaven has [under Heaven's] not having [any object/entity $x$ ], and 3) dao is [under Heaven's] not having [any object/entity $\mathrm{x}$ ]," into a statement of quite a different sort, the meaning of which is not altogether clear.

We may well want to compare the three-fold full intent of "dao wu" as described above with an English expression which states "dao is nonBeing," but this would be a comparison and not a translation. That is to say, we cannot simply substitute English "non-Being" for Chinese "wu." We must first make explicit all that is implicit in the abbreviated Chinese expression, then literally translate the whole Chinese expression, before making a comparison. The same is true for other English terms such as "non existence," "nothing" or "void." None of these terms syntactically matches Chinese "you" and "wu" well enough to be helpful in making literal translation. Without careful literal translation, accurate comparison of the meaning of philosophical statements coming from two different languages is not possible.

Our own sense is that the three-fold full meaning of "dao wu" 
detailed above is compared better with the meaning of the English statement, "dao is Being," than with the meaning of the statement, "dao is non-Being." But, again, this is a comparative assessment based upon careful literal translation having made explicit all that is implicit in the Chinese abbreviated expression. We could hardly take "is Being" as a translation match for "wu" in "dao wu."

We would offer a second example of an expression using " $w u$ " which illustrates the importance of making explicit in English a location which does not have $(w u)$ an object/entity and an object/entity which is not had. Kong $\mathrm{Zi}$ (Confucius) observed that dao did not prevail in the everyday world of his time. ${ }^{111}$ One could express this observation as: tianxia wu dao (literally, under Heaven does not have dao)." However, given Chinese actualist discernment of dao to the effect that under Heaven does have dao, we have to consider the above expression to be an abbreviation of a longer statement.

We would suggest that an expanded statement of Kong Zi's observation is a combined expression which has "under Heaven has dao" (tianxia you dav) as the first part. Dao, then, is discerned as had (you) or present (zai) in the world. As regards the second part of the combined expression, what object/entity serving in syntactic object role is not had $(w \cdot u)$ ? We would suggest that "manifestation of dao" (dao zhi shixian) is the object/ entity not had and that "dao," in this context, is an abbreviation for "manifestation of dao." In the first part of the combined expression, "under Heaven" is taken as a location serving in syntactic agent role. The same is true for the second part of this combined expression, that is to say, under Heaven is taken as the location which does not have manifestation of dao. Taken together, we have a two-part combined expres. sion: under Heaven has dao, but under Heaven does not have manifestation of dao (tianxia you dao er tianxia wu dao zhi shixian). The expression "tianxia wu dao" abbreviates this expanded expression by leaving implicit the first part of the combined expression and abbreviating "manifestation of dao" as simply "dao." Making explicit what is implicit as well as a careful literal translation of the whole combined expression 
renders clear the meaning of enigmatic "tianxia wu dao." Kong Zi's remedy for "under Heaven's having $d a o$, but not having manifestation of dao" is had in the oral-written record of the way of sage-kings, but not yet fully manifest in the world.

In summary, without making explicit the implicit locations and objects/entities linked with locations in abbreviated Chinese expressions, careful, literal translation of " $y o u$ " and " $w u$ " expressions is not possible. Further, while substitution of a Western philosophical term such as "Being" or "non-Being," "existence" or "non-existence," "something" or "nothing," may perhaps work in one context or another, if at all, notional substitutions of Western philosophical terms will not work consistently in all contexts. Zhang Dainian in his discussion of "you" and " $w u$ " in traditional Chinese philosophy states, and makes clear in his examples from classical texts, that "you" and "wu" have no constant determination and are concepts having many layers of meaning. ${ }^{112}$ In our view, we are much better off with a literal translation of "you" and "wu" which allows a location and an object/entity had or not had in that location to inform us more fully as to what their linkage or lack of linkage. linguisticially expressed using "you" or "wu" construction, means in any one context. Then we can compare specific contexts of "voul" and "wu" with certain Western philosophical notions.

One final point needs be emphasized. In our efforts to make clear the location and object/entity linked or not linked by "you" and "wu," we have labored hard throughout this volume to avoid in each instance use of the quite ambiguous English expressions "there is/exists" and "there is not/does not exist" to translate Chinese "you" and "wu" respectively. Conversion of a "you" or "wu" syntactic construction to a "zai" or " $b u$ zai" construction brings to the surface an implicit object/entity and an implicit location. By making explict an implicit object/entity and an implicit location in a "you" or " $w u$ " expression, and by literally transiating "you" and "wu" as "have" and "not have," respectively, one can invariably avoid English "there is/exists" or "there is not/does not exist" in translation. By avoiding "there is/exists" or "there is not/does not 
exist" in English, one avoids substituting existential claims in English translation for "you" or "zai" expressions in Chinese which do not make existential claims.

We close this section with four examples taken from classical Chinese texts with 1) literal translation of Chinese "you" and "wu" of the sort we have demonstrated above, and with 2) notional substitution of Western philosophical terms offered by A. C. Graham. (In the literal translations first offered, we supply in brackets the locations, object/ entities and other terms left implicit in Chinese.)

He Yan (Ho Yen) (died A.D. 249) has made much the same sort of expression regarding dao as we have in our first expression above "under Heaven has dao and dao does not have (any object/entity)" (tianxia you dao er dao wu)":

Fu dao zhe, wei wu suo you zhe ye.

1. As for dao, [it is that which] alone does not have that which is had.

2. The dao is only that in which there is not anything that there is." 113

A statement by Wang Bi (Wang Pi) (A.D. 226-249) elaborates upon this point:

Dao zhe, wu zhi cheng ye. Wu bu tong ye. Wu bu you ye. Kuang zhi yue dao. Mo ran wu ti, bu ke wei xiang.

1. As for dao, [it is] a name for not having $(w u)$. [Under Heaven] does not have $(w u)$ [that which dao] does not penetrate. [Under Heaven] does not have $(w u)$ [that which] does not follow [dao]. [We] call it dao. [Dao] is still and does not have $(w u)$ a body and cannot be made into an image.

2. The Dao is a term for Nothing. Since there is nothing it does not pass through and nothing which does not follow it, it is called by metaphor the Dao (Way). It is

Ixxiv 
still and has no body, and cannot be treated as an image. ${ }^{114}$

Another well-known passage comes from Lao $Z i$ :

San shi fu gong yi gu, dang qi wu you che zhi yong. Yanzhi yi wei qi, dang qi wu you qi zhi yong. Zao hu you yi wei shi, dang qi wu you shi zhi yong. Gu you zhi yi wei li, wu zhi yi wei yong.

1. Thirty spokes share one hub. Precisely where [the wheel] does not have $(w u)$ has $(y o u)$ the usefulness of the wheel. Clay is taken to make a vessel. Precisely where [the vessel] does not have (wu) has (you) the usefulness of the vessel. Chiseling doors and windows is undertaken to make a house, precisely where [the house] does not have $(w u)$ has the usefulness of the house. Thus, the advantage which can be taken of having [is] the usefulness which can be made of not having.

2. Thirty spokes share one hub; it is just where it does not exist that the wheel is useful. We turn clay to make a vessel; it is just where it does not exist that the vessel is useful. We chisel out doors and windows to make a house; it just where it does not exist that the house is useful. Therefore we draw advantage from them where they exist, use them where they do not exist. ${ }^{115}$

Our last example comes from the Xiang Xiu-Guo Xiang Commentary on the Zhuang $Z i$, which Feng Youlan translated in the late 1920s:

Fei wei wu bu de hua er wei you ye, you yi bu de hua er wei wu yi. Shi yi you zhi wei wu, shi qian bian wan hua, er bu de yi wei wu ye. Bu de yi wei wu, gu zi gu wu wei you zhi shi er chang cun ye. 
1. [It] is not only that not having (wu) is not able to transform to become having (you). Having (you) also cannot transform to become not having $(w u)$. For this reason, if [we] take becoming things which pertains to having (you), even if [things] undergo a thousand changes and ten thousand transformations, still we do not acquire one which becomes not having $(w u)$. Because we do not acquire one which becomes not having, from old we do not have $(w u)$ a time of not yet having (you). [Having] always is present (cun).

2. It is not only that Nothing cannot be transformed into Something; Something also can no longer be transformed into Nothing. Therefore Something is so constituted that, although it alters and transforms in thousands and myriads of ways, it can never become Nothing. Because it can never become Nothing, from the remotest past there has never been a time before there was Something and it will always continue. ${ }^{116}$

\section{One Small Change: Feng Youlan's Philosophical Terms in English Translation}

We have observed in detail how, in traditional and customary use, expressions "you dao" ([under Heaven] has dao) and "dao zai" (dao is present [under Heaven]) mirror each other, semantically and syntactically. In both expressions, "tianxia" (under Heaven or all under Heaven) is an implicit location in which an action, referred to with either "you" or "zai" serving in syntactic action role, takes place. In the discussion which follows, we continue to be concerned with expressions which take "tianxia" (under Heaven or all under Heaven) as an implicit location.

In the twentieth century Feng Youlan made one small change: for statements with "tianxia" (under Heaven) as an implicit location, Feng took "you" (have, having) statements as equivalent to "cunzai" (exist, Ixxvi 
existence) statements, rather than as equivalent to "zai" (is present in) statements as in traditional Chinese philosophy. ${ }^{117}$ It is quite simply this one small change, having endless ripple effects, that has caused a great stir.

Feng knew well what he was doing in making this one small change. His purpose was to explore how well and how far this one shift could go in presenting traditional Chinese actualist discernment by using terms of contemporary Western philosophy which cluster around the notion of existence. Many have criticized Feng for making this shift; others have marveled at just how far he was able to go with it.

In matching Chinese "you" and "cunzai," the particular version of English "existence" which Feng chose for this matching was that of New Realism, a school of thought which came into its own during the early 1920s when Feng Youlan was pursuing his doctoral studies at Columbia. ${ }^{18}$ Applying New Realist understanding of "existence" to the modern Chinese expression "cunzai" and matching "cunzai" with Chinese "you," Feng found what he discerned as a bridge, crossing great waters, linking Chinese traditional actualist philosophy and Western New Realist philosophy. Across this bridge, Feng willfully traveled to open up and to engage insights of traditional Chinese philosophy within contemporary, world-wide philosophical discourse.

We cannot explore all the ramifying shifts in Chinese philosophical thinking that result from this one small change of aligning Chinese "you" with "cunzai" instead of "zai" in expressions which have "tianxia" (under Heaven) as an implicit location. We will, however, put our attention to four areas in which clusters of important changes occur. In doing so, the stage is set for more detailed discussion of these changes in essays of this volume.

We consider first how Feng is able to match all "you" statements with "cunzai" statements when an implicit location is "under Heaven" or "all under Heaven." We set forth in Sub-section Three above the ambiguity of the Chinese abbreviated expressions "you" and "you $x$ " under two conditions: namely, when the implicit location of "you" is "under 
Heaven" or "all under Heaven" (tianxia), and when object/entity "x," which may or may not be implicit, is an actual event/thing in the everyday world. Under these conditions the expressions "you" and "you x" may be taken as abbreviations for either 1) under Heaven has $\mathrm{x}$ (tianxia you $x$ ) or 2) under Heaven has the existence of $x$ (tianxia you $x$ de cunzai). The first of these longer expressions converts to a "zai" expression, i.e. $\mathrm{x}$ is [present] under Heaven, and is not an existential claim. The second of these longer expressions converts to a "cunzai" expression, i.e. $\mathrm{x}$ exists under Heaven, and is an existential claim. When the above two condi. tions obtain, some consider that statements 1) and 2) are equivalent. That is to say, under the two conditions above, some deem "you" statements as equivalent to "cunzai" statements.

However, what happens when "dao" or "li" is taken as an object/ entity which is said to be had (you) or present in (zai) in the implicit location "under Heaven" or "all under Heaven"? Strictly speaking, "cunzai" applies only to actual events/things in the everyday world. If dao or $l i$ assumes the role of object/entity in syntactic object role, which may or may not be implicit, in the abbreviated expressions "you" and "you $x$ " or their longer forms, can these expressions also be converted to "cunzai" expressions? That is to say, are these "you" expressions with dao or $l i$ also deemed equivalent to "cunzai" expressions? If so, how?

In matching Western New Realist discernment with Chinese actualist discernment, Feng Youlan not only brings withh him New Realist understanding of existence, but also "subsistence." English terms "subsist" and "subsistence" come from Latin: "sub" meaning "under" and "sistere" meaning "to place, to stand." English "hypostasis" has a similar derivation from Greek: "hypo" meaning "under" and "histanai" meaning "to stand, to make to stand." To "subsist" is 1) to be, to exist, 2) to continue to be or exist, 3) to inhere (in) and 4) in philosophy, to be logically conceivable and, hence, to hold true. ${ }^{119}$ Bearing these several senses of "subsist" in mind, we could say that "subsistence" is a variant of "existence" which is continuous, inheres in something else, and is logically conceivable and, therefore, true. In short, subsistence is continu- 
ous, underlying, inhering, logically true existence.

Feng uses two Chinese terms, "qianyou" and "qiancun," to translate "subsistence." Chinese "qianyou" is used in a manner syntactically parallel with Chinese "you," while "qiancun" is used in a manner syntactically parallel with Chinese "cunzai." We shall have more to say about these syntactic parallels in a moment. First, as regards the terms themselves, Chinese "qian" means "to hide, to conceal." Keeping our literal English translation of Chinese "you" as "have, having," "qianyou" may be translated literally into English is "hiddenly have, hidden having." In the Chinese term "qiancun," "cun" is an abbreviation of "cunzai." Literal English translation of "qiancun" is "hiddenly exist, hidden existence." We carry on with these literal translations for the moment.

With the New Realist notion of subsistence, the above problem regarding "you" statements with dao or $l i$ as an object/entity said to be had or present in all under Heaven appears to be solved. By matching Western "idea" or "form" (in the Platonic or New Realist sense) with Chinese $l i$ (pattern, principle), li may be said to "subsist" (li qiancun). In other words, in New Realist terms, 1) actual events/things in the everyday world exist (cunzai) and 2) li subsist (qiancun). The first expression in the form " $x$ cunzai" converts to a "you" expression of 'you $x$ de cunzai" (has the existence of $\mathrm{x}$ ). The second expression in the form of "x qiancun" converts to a "you" expression of "you $x$ de qiancun." In this manner, whether actual events/things serve as an object/entity in syntactic object role, or dao or li serve as an object/entity in syntactic object role, all "you" expressions are deemed to convert to "cunzai" or "qiancun" statements. That is to say, all "you" statements are deemed to make either existential claims or subsistential claims. Further, when the implicit location is "under Heaven" or "all under Heaven," all "zai" expressions are deemed equivalent to either existential or subsistential claims.

This is a substantive shift as regards the import of traditional Chinese philosophical statements. Specifically, as regards the meaning of "you," its purpose is no longer understood to link an object/entity with a location 
as a way of expressing discerned relationship. (See Sub-section Three above.) Traditional non-assertiveness as regards "you" and "zai" expressions is abandoned as an object/entity serving in syntactic agent role is asserted to "exist" in itself. That is, instead of saying "(the world) has a book" or "a book is present (in the world)," one asserts "book exists." Or, instead of saying "(the world) has me" or "I am present (in the world)," one asserts "I exist." So also, instead of saying "(the world) has dao" or "dao is present (in the world)," one asserts "dao subsists." 120

Before turning to a second cluster of shifts which come with Feng's matching "you" with "cunzai" (instead of "zai"), we want to explore a bit further syntactic constructions using 1) "you" and "cunzai" and 2) "qianyou" and "qiancun." Syntactic constructions using these terms in mirror-like ways parallel traditional and customary syntactic constructions using "you" and "zai" in mirror-like ways. For example, the place or general location which has (you) actual events/things Feng calls "actuality" (shiji). The place or general location which hiddenly has (qianyou) li (pattern, principle) Feng calls "truth" (zhenji). For Feng, just as "you" statements convert into "cunzai" statements, so also "qianyou" statements convert into "qiancun" statements. Hence, "actuality has events/things" (shiji you shiwu) converts to "events/things exist in actuality" (shiwu cunzai yu shiji). Similarly, "truth hiddenly has $l i "$ (zhenji qianyou li) converts to "li hiddenly exists in truth" (li qiancun yu zhenji). ${ }^{121}$

Bearing in mind Feng Youlan's intentional matching of "you" (have, having) with "cunzai" (exist, existence), why do we not translate both "you" and "cunzal" with English "exist" or "existence" in this volume? Why do we maintain a literal translation of "you" as "have" or "having"? Similarly, given Feng Youlan's deliberate intention to translate the New Realist understanding of "subsist" into Chinese using both "qiancun" and "qianyou," why do we not translate both these Chinese terms back into English with "subsist" or "subsistence"? These are important questions and after considerable painstaking effort in making several alternative translations of the papers of this volume, our decision $\operatorname{lxxx}$ 
to work with literal rather than notional translations for "you," "qiancun" and "qianyou," is deliberate for the following reasons.

First, as regards "you," which for Feng is equivalent to "cunzai" (exist), we have found that although Feng conceptually matches "you" with "cunzai," the syntactic constructions in which Feng puts "you" are traditional and customary syntactic patterns. In addition, other scholars whose papers appear in translation in this volume continue to use "you" in traditional and customary patterns. Therefore, to be consistent with one translation of "you" through this volume, even though Feng Youlan notionally matches "you" with "cunzai," we continue to translate "you" literally in its traditional and customary sense of "have" or "having."

Second, Feng Youlan uses "qianyou," the variant of "you" which applies to dao or $l i$, in traditional and customary patterns of "you" as well. Having decided for literal translation of "you," it has been helpful to go with a literal translation of "qianyou" (hiddenly have, hidden having) so that semantic and syntactic parallels between "you" and "qianyou" are readily apparent. Similarly, because "qianyou" statements, with dao or $l i$ serving as an object/entity in syntactic object role, convert to "qiancun" statements, with dao or $l i$ as an object/entity serving in syntactic agent role, having decided for literal translation of "qianyou," we opt also for literal translation of "qiancun" (hiddenly exist, hidden existence). Chinese "cunzai" translates Western "exist" or "existence." So also, "cun" in "qiancun" translates Western "exist" or "existence." Both "cunzai" and its shortened form "cun" we return back to English as "exist" or "existence."

In summary, in order that we may present obviously in English translation syntactic parallels between "qianyou" and "you" as well as "qiancun" and "cunzai," we return "cunzai" back to English as "exist," but we do not return "qianyou" and "qiancun" back into English as "subsist" or "subsistence." Without presentation of these syntactic parallels in English, arguments of these papers can not be adequately conveyed in translation. As one works into Feng Youlan's use of these terms as well as that of his critics, one will find literal translation of these 
terms essential for grasping semantic and syntactic inter-relationships among them.

Third, in exploring Feng's conceptual match of "you" with "cunzai" while yet using syntactic patterns of "you" and "zai," literal translation of these terms is helpful in bringing to light "where the new shoe fits" and where it does not. That is to say, literal translation of these terms allows one to observe closely where the match between "you" and "cunzai" aligns with traditional and customary use of "you" and "zai" and where it does not. In so doing, one comprehends where Feng's use of New Realist understanding of existence and subsistence aligns with traditional Chinese actualist discernment and where it does not.

Finally, in practical terms alone, with regard to the essays in this volume, we simply have found literal translation of 1) "you" and 2) "qianyou" or "qiancun" for more efficient and requiring far less explanatory notation in English than notional matching of these terms with English 1) exist, existence and 2) subsist, subsistence, respectively, even though Feng Youlan did have those notional matches in mind. Importantly, we have found that literal translations of these terms does not detract from but rather illumines Feng Youlan's own matching of "you" and "cunzai."

A second important area of shifting that comes with Feng Youlan's matching of "you" and "cunzai" concerns $l i$ (pattern, principle). In Western New Realist philosophy, ideas subsist and events/things exist. As we have said, Feng Youlan matches New Realist "idea" with Chinese $l i$; so also Feng matches Western "event/thing" with Chinese shiwu. Hence, li "subsist" (qiancun) and shiwu "exist" (cunzai). In addition, an idea, in New Realist understanding, is universal and an event/thing is particular. Feng also applies these notions of universal and particular to $l i$ and shiwu. $\mathrm{He}$ does this using the Chinese term "gongxiang" for universal and the Chinese term "shuxiang" for particular.

The Chinese term "xiang" refers to "look, appearance" and has been used by Buddhists to translate into Chinese the Sanskrit term "laksana" meaning "quality/ies of a thing." 12 In his New Lixue, Feng distinguishes 
between "shared quality/ies" (gongxiang) or universals which are $l i$, that by which a certain thing is a certain thing, and "different quality/ies" (shuxiang) or particulars, which are shiwu (events/things). In Feng's usage, "gongxiang" is similar to Chinese "pubian," the latter meaning "general, common." Both terms, in Feng's thinking, translate English "universal." Feng uses "shuxiang" in a manner similar to Chinese "teshu," the latter meaning "specific, unique." Both these latter terms, in Feng's thinking, translate English "particular."

Feng's matching of Chinese $l i$ with Western "idea" or "universal" brings a significant shift concerning $l i$ in that Chinese actualist discernment of one-to-one correspondence between pattern in heart-mind and pattern discerned in events/things of the actual world takes on a new understanding. (See Sub-section Two above.) Because a universal may pertain to more than one event/thing, Chinese actual discernment of $l i$ shifts from one $l i$ for one event/thing to one $l i$ for more than one event/ thing. Several philosophers in their essays in this volume will attend to problems, insights, gains, and losses which accompany Feng's matching of Western "idea" or "universal" with Chinese $l i$. We leave further exploration of ramifications, resulting from Feng's matching of "you" and "cunzai," which concern $l i$ and Chinese actualist discernment of $l i$, to these essays.

A third area of shift resulting from Feng's matching of "you" and "cunzai" concerns $t i$ (substance) and yong (function). As discussed in Sub-section Four above, in Song-Ming $R u$ discernment $l i$ is discerned as substance and events/things are discerned as function. Actual events/ things as function in shape-and-form in space-and-time are discerned to manifest substance in the everyday world. In linguistic expression, events/things are said to have (you) $l i$. In this linguistic expression, events/ things are considered a location serving in syntactic agent role and $l i$ is considered as an object/entity serving in syntactic object role. Recalling He Yan's statement, cited at the end of Sub-section Five above, that "as for dao, it is that which alone does not have that which is had" (fu dao zhe, wei wu suo you zhe ye), ${ }^{123}$ we may say also of $l i$ (pattern, 
principle) that $l i$ is that which alone does not have that which is had. That is to say, in linguistic expression, although "under Heaven" or "all under Heaven" or "all actual events/things" taken as a location serving in syntactic agent role may be said to have $d a o$ or $l i$, neither dao nor $l i$ when taken as a location serving in syntactic agent role has anything else. In effect, this is to say that neither dao nor $l i$ can be taken as a location. For in Song-Ming $R u$ discernment, dao and $l i$ are not function but substance.

In our discussion of "you" and "zai" in Sub-section Three above, we observed that in Chinese a locational suffix term such as "shang" (above), or "xia" (below), "zhong" (within), "xian" (before or in front of), and so forth, may be added to an event/thing to indicate explicitly that an event/thing serves as a location in a linguistic expression. It is important to comprehend in English translation that Chinese expressions such as "before things" (wuxian) or "above things" (wushang) or "within things" (wuzhong) do not indicate a place separate from things. Rather, the addition of a locational suffix indicates a sub-location within a location. Hence, in the expression "in front of" the door, the door is the location, and "in front of" is a sub-location within the location of the door. The same is true for the expression "above" the door, and so forth.

In Song-Ming $R u$ terms, Chinese actualist discernment has been expressed as "li is [present] (zai) before things" (li zai wuxian), or "li is [present] above things" (li zai wushang), or " $l i$ is present within things" (li zai wuzhong). When $l i$ is described as present "before things" or "above things" or "within things," things is taken as a location, and "before" or "above" or "within" is a sub-location of that location (things). That is to say, "before" or "above" is not a place separate from things as a location. Each of these Song-Ming expressions articulates discernment of relationship between $l i$ and things, between substance and function. Expression of this discerned relationship differs slightly as regards the specific sub-location (above, below, or within) with respect to things which are taken as a location, serving in syntactic agent role, that is said to have $l i$.

However, when Feng Youlan states, on the one hand, that truth has

Ixxxiv 
li (zhenji you li) or that li hiddenly exists in truth (li qiancun yu zhenji) and, on the other hand, actuality has events/things (shiji you shiwu) or that events/things exist in actuality (shiwu cunzai yu shiji), an important shift has taken place. In linguistic expression, the location that is said to have $l i$ is no longer actual events/things in the everyday world as in SongMing $R u$ expressions. Rather, in Feng Youlan's expression, truth is taken as a location, serving in syntactic agent role, and is said to have $l i$; similarly, actuality is taken as a location and is said to have actual events/things.

In this manner, Feng in his New Lixue system does something quite new with Song-Ming expressions such as "li is before events" (li zai shi xian) and "li is above events" (li zai shi shang). He considers "before" or above" not as a sub-location within a larger location "events," but rather as a location altogether separate from events. In this way, Feng enables the Chinese language to discuss a "two world" theory: a world of $l i$ and a world of actuality quite separate from each other and having, in fact, no point of direct contact. This, then, makes for what could be called a "docetic" view of $l i$ and events/things. ${ }^{124}$ Essays of Sections Three and Four of this volume, in particular Professor Chen Lai's essay, will explore in detail ramifications with regard to Feng's re-working Song-Ming terms to express a "two world" theory.

Finally, we turn to a fourth importart area of change, resulting from Feng's matching of "you" and "cunzai," which concerns the purpose for which one engages in philosophy. Chinese actualist discernment and description of the world would seem to lead in one direction, while Platonic and New Realist discernment and description of the world would seem to lead in another direction. This does not necessarily mean that what is discerned in itself is different. Interestingly, Feng Youlan himself fathomed that even though these two types of Chinese and Western philo. sophical discernment and description lead in different directions, still at important points some of what is discerned and described on both sides comes extremely close to the other-one could say, even touches the other, only to part on another path. It is this convergence at significant points that fascinated Feng Youlan and moved him to explore, deftly 
and brilliantly, whether all could converge between these philosophical views. Our own view and that of many of Chinese philosophers today is that while there is convergence at significant points, divergence between these two philosophical views carries the day.

Those of Chinese actualist discernment have discerned actual events/ things in the everyday world as functional extensions into shape-and. form and into space-and-time of substance $l i$ (pattern, principle). As concrete, durable extensions in shape-and-form in space-and-time of li, actual events/things are considered to manifest presence of $l i$ in the everyday world. $L i$ is present $(z a i)$ in the world. Actual events/things, which manifest li present in the world, exist (cunzai) in the world. In this view, we could consider "cunzai" (existence) as a concrete, durable variation of "zai" (presence in). That is to say, notionally, "existence" is modelled on "presence" and is an extended in shape-and-form in spaceand-time version of presence. In this view, "presence in" (zai) is more primary than "existence" (cunzai). This is illustrated linguistically in that "zai" may apply both to $l i$ and to events/things. "Cunzai" applies only to events/things. That is, we can state that $l i$ is present in the everyday world in actual events/things under Heaven, and we can state that events/ things are present (zai) in the world. However, "cunzai" as a variation of " $z a i$ " applies only to events/tlings as function which manifests $l i$ as substance present in function.

Chinese actualists seek to manifest perfectly-in shape-and-form in space-and-time in existing (cunzai) actual events/things in the everyday world-li which is discerned as already present in (zai) actual events/things in the everyday world. As we have previously described, their efforts have been "to bring the grain out in the wood" of everyday living. As $d a o$ or $l i$ is discerned as the way to life, harmony, peace, and what is good, then knowing dao or $l i$ is for the purpose of manifesting dao or $l i$ in activity in the world. The more perfect and complete knowing of $d a o$ or $l i$ is, the more perfect and complete manifestation of dao or $l i$ in practice is. In this manner, life, harmony, peace, and all that is good prevails here in the world.

Lxxxvi 
By contrast, Platonic and New Realist discernment and description of idea and events/things in the world come from the other way around. "Existence" pertains to events/things in shape-and-form in space-andtime. "Subsistence" is a rarefied, without shape-and-form, without spaceand-time, variation of "existence." This is to say, notionally, subsistence is modelled on "existence," and is a more rarefied form of existence which pertains to ideas or forms and is objective, true, and pure. Actual manifestation of idea in shape-and-form in space-and-time in existence is discerned as always imperfect, incomplete, and problematic. Therefore, Platonic and New Realists seek to pass through and beyond existence, the realm of actual manifestation of ideas and universals, to the realm of idea or form which subsists. What is treasured is perfect idea or form, which can be contemplated, known, analyzed, and appreciated in itself. As Bertrand Russell has described it, the world of ideas or universals is unchanging, correct, precise, pure, bringing happiness and delight to mathematicians, logicians, metaphysicians and all those who love life and find it beautiful. ${ }^{125}$

To be sure, for early Western Realists such as Plato and Socrates, to know idea or form (universal) is to enliven idea or form in practice which is virtue. Some scholars have discerned convergence here between China and the West as regards knowing idea/form and knowing $l i$ (pattern, principle) in that both lead to moral practice. Yet, clearly, the road which leads to unity of knowing and doing is discerned and described from quite different directions in these two traditions, although, as we have said, there are striking points of convergence which Feng Youlan mightily endeavored to extend.

In the above analysis, our concern has not been to evaluate which is the better or more accurate discernment or description of our world and our engagement in it. Rather, our concern has been with accurate translation, paying attention to differences of philosophical discernment and of linguistic expression used to convey philosophical discernment. Our effort in transiation has been to allow these differences to speak in one and the same language and not to cover these over with notional matches 
which, while suggesting much in common, hinder more analytic comparison which would bring true similarities and differences to light.

We close this sub-section on Feng's philosophical terms in English translation with a consideration of a few remaining expressions needing further clarification before embarking upon the essays.

The Chinese term which we translate into English as "actual" is shi (actual, solid, practical, honest, fruit). Shi (actual) combines with shi (event, matter, business) in shishi (factual). In terms used by Feng Youlan, shi (actual) occurs in shiji (actuality), shixian (manifest in actuality), shicun (actually exist, actual existence), shiyou (actually have, actual having) and in combined expressions such shiji de shiwu (events/ things in actuality), shiji de cunzai (existence in actuality), shiji cunzai de shiwu (events/things existing in actuality).

Interestingly, but not surprisingly, in Feng's and others' use, shi (actual) also occurs in the Chinese translation "shizai" of Western "real" in New Realism (Xin Shizailun) or Plato's Realism (Shizailun). Shizai literally translated means "actually present (in)." As a translation for Platonic and New Realist "real," shizai is somewhat problematic. Feng Youlan holds that li are "real" (shizai) but do not exist (cunzai) in actuality (shiji). The shi of "real" and the shi of "actuality" are one and the same. Hence, while in English it may make sense to say that $l i$ are real but do not exist in actuality, in Chinese, we have "li shizai er bu shicun" which, literally translated is " $l i$ is actually present but not actually existing," which to some may be contradictory. One way around using Chinese "shizai" to translate English "real" is to use Chinese "zhen" for English "real," as Professor Chen Lai does when he talks of $l i$ as "true but not actual" (zhen er bu shi). ${ }^{126}$

As regards the terms "shiji" (actuality) and "zhenii" (truth), Chinese " $j i$ " has the sense of border, boundary, region, place. ${ }^{127}$ As we have set forth above, syntactic constructions using "cunzai" parallel traditional and customary syntactic constructions using "zai." An event/thing exists (cunzai) in a place/location and a place/location has (you) existence of an event/thing (shiwu de cunzai). Thus, in Feng Youlan's presentation,

Ixxxviii 
events/things exist (cunzai) in actuality (shiji) and actuality has existence of event/things. Similarly, $l i$ hiddenly exist (qiancun) in truth (zhenji) and truth has hidden existence (qianyou) of $l i$ (pattern, principle).

Further in this regard, Feng Youlan speaks of "the world of $l i$ " (li shijie) as another term for "zhenji" (truth) and of "the world of facts" (shishi shijie) as another term for "shiji" (actuality). However, in a different kind of discussion, Feng speaks of four spriitual worlds (jingshen shijie), abbreviated as "jingjie." In his theory of spiritual worlds, Feng is not concerned with a theory of existence and subsistence as he is in his discussion of the world of $l i$ (pattern, principle) and the world of shish $i$ (facts) or shiwu (events/things). Rather, in his theory of spiritual realms, Feng is concerned with different types of knowing or levels of knowing.

Although some translators have translated shiji as "realm of actuality" and zhenji "realm of truth," in this volume, in order to differentiate between 1) Feng's ontological discussion of two worlds (shijie) of $l i$ (pattern, principle) and shi (facts/events) and 2) Feng's spiritual discussion of four worlds (shijie), we have chosen to translate shiji simply as "actuality" and zhenji simply as "truth." English "world" translates Chinese "shijie" as is the usual custom. Hence, Chinese "shiji shijie" and "zhenji shijie" are rendered in English as "world of actuality" and "world of truth," respectively. This leaves the English term "realm" for Feng's four "spiritual realms" (jingjie).

As regards the term "cunzai" (exist, existence), Feng uses cunzai in two ways: 1) for "exist" in actuality (shiji de cunzai) and 2) for "exist" more generally, including "hiddenly exist" (qiancun [subsist]). Feng occasionally states that "li exists" (cunzai). However, when Feng wants to state narrowly that $l i$ hiddenly exist, he will use the Chinese term "qiancun" to contrast with "cunzai," which is used more narrowly of events/things in actuality. In some instances authors of this volume use "shiyou" and "shicun" to refer narrowly to "actual having" and "actual existence" in contrast with "qianyou" (hidden having) and "qiancun" (hidden existence). Literal English translation of these terms renders the conceptual parallels between these sets of terms readily apparent. 
As stated in Part One of this Introduction. Feng Youlan's four main concepts in this volume are translated into English as follows: 1) li (pattern, principle), qi (energy-matter), daoti (embodiment of dao [Way]), and daquan (great whole). In Feng Youlan's New Lixue system, the great whole includes both shiji (actuality) and zhenii (truth). Because shiji has (you) events/things and zhenji has (you) li, the great whole may be considered the whole of all "you" (have, having), including both "shiyou" (actual having) pertaining to events/things and "qianyou" (hidden having) pertaining to $\mathrm{li}$.

We have earlier discussed the Chinese linguistic custom of abbreviating expressions with you, leaving implicit the location which has (you) an object/entity and, sometimes the object/entity had as well. Often times in "you" constructions, no specific location is implied, but, rather, the whole of all having (you) is implied and taken as a general location which is said to have (you) an object/entity currently in our attention. In an effort to make explicit what abbreviated Chinese expressions of the form "you" and "you $x$ " keep implicit, throughout this volume we use the expression "the greater world" for Feng's "great whole" which includes both actuality (shiji) and truth (zhenii). When no specific location of having (you) is mentioned or known in abbreviated Chinese expressions of the form "you" or "you x," "the greater world" is supplied in brackets as a general location.

To give example to our use of "the greater world" to refer to the great whole taken as a general location in "you" constructions, we shall turn momentarily to Feng Youlan's well-known proposition which contains two instances of abbreviated "you x." First, however, we do well to summarize which Chinese terms we translate as English "is" in this volume.

We have discussed our translation of Chinese "zai" as "is in" or "is present in," where "is" in these expressions is what we have called "floppy is," that is, "is" here makes no existential claim but serves, rather, by requirement of English syntax, as a verbal connective. ${ }^{128}$ Additionally, we have detailed in Sub-section Five above that by making explicit an 
implicit location of having (you) in abbreviated expressions of the form "you" and "you x," we may avoid expressions such as "there is/are" or "there exists/exist" in English as translations of Chinese you. Chinese "wei" meaning "to do, to make, to act," which is used to indicate that a certain object/entity takes on a certain role, we translate into English as "is discerned as," "is considered as," "is taken as," and so forth, depending on context. ${ }^{129}$ Here "is" is required by English syntax for passive forms of verbs "discern," "consider," "take," and so forth. What remains to translate into English as copulative "is" is simply Chinese shi (is), formerly a pronoun meaning "this here," which has become in modern vernacular Chinese a verb which serves as a verbal copula, much as English copular "is" does, connecting two nouns or two nouns phrases.

We are now able to appreciate more fully some of the ambiguities and subtleties of Feng Youlan's well-known proposition, of the first set of four sets of propositions which structure Feng's New Lixue, putting the whole of the above linguistic analysis of Feng's philosophical terms and expressions to work. In its abbreviated form, this proposition in Chinese reads: "you mouzhong shiwu bi you mouzhong shiwu zhi suo yi wei mouzhong shiwu zhe." Before we offer three possible English translations of this proposition, we do well to consider its basic form. The proposition connects two expressions of the "you x" type which we have discussed above. The two "you $\mathrm{x}$ " expressions are connected by Chinese "bi" (must). In basic form, the proposition reads, "have (you) a, must have (you) b" or "if have a, then must have b."

Due to ambiguity in two places, the proposition may be read or understood in three possible ways. First, the proposition does not state explicitly the location which serves in syntactic agent role in either part of the proposition. Second, it is not clear whether the implied location which serves in syntactic agent role in the first part of the proposition is the same implied location which serves in syntactic agent role in the second part of the proposition.

In this volume, when translating this well-known proposition of Feng's New Lixue, generally (unless otherwise indicated), we have taken 
"the greater world" as the implied location serving in syntactic agent role for both parts of the proposition. In this manner, making the implicit location explicit, we translate the proposition as: "If [the greater world] has events/things of a certain kind/type, [the greater world] must have that by which events/things of a certain kind/type are discerned as events/ things of a certain kind/type." This is the most inclusive manner in which this proposition may be read.

However, the implied location which serves in syntactic agent role may be understood as different in the two parts of the proposition. Thus, "actuality" may be taken as the implied location serving in syntactic agent role in the first part of the proposition, and "truth" may be taken as the implied location serving in syntactic agent role in the second part of the proposition. In this manner, the proposition would read: "If [actuality] has events/things of a certain kind/type, [truth] must have that by which events/things of a certain kind/type are discerned as events/ things of a certain kind/type." This second reading of the proposition is a more narrow understanding of the proposition which does not in any way conflict with the first more inclusive reading of the proposition. This second reading is closest to Feng's usual understanding of the proposition.

A third reading of the proposition takes "actuality" as the location which serves in syntactic agent role in the first part of the proposition, and "actual events/things of a certain kind/type" as the location which serves in syntactic agent role in the second part of the proposition. In this manner, the proposition would read: "If [actuality] has events/ things of a certain kind/type, [actual events/things of a certain kind/type] must have that by which events/things of a certain kind/type are discerned as events/things of a certain kind/type." This third reading of the proposition also does not conflict with the first more inclusive reading and, like the second reading, is a more narrow understanding of the first. This third reading is probably not Feng's usual understanding of the proposition, but may be closer to a traditional and customary Chinese understanding of this proposition.

In order to accomodate both Feng's understanding of the proposi- 
tion and a traditional Chinese understanding of the proposition, we have presented the more inclusive reading of the proposition (the first presented above) throughout this volume. Notice that without literally translating Chinese "you" as "have/having" (instead of "exist/existence" or "being") in English, and without taking account of possible different locations serving in syntactic agent role in each part of the proposition, we cannot render clearly or insightfully into English the ambiguity of this proposition nor its different readings, all of which are essential to understanding and evaluating Feng's New Lixue, as essays in this volume will demonstrate.

\section{NOTES}

1. Feng Youlan is in Pinyin romanization; Fung Yu-lan is in Wade-Giles romanization. Throughout this volume of essays by philosophers from the People's Republic of China, we use the Pinyin romanization. However, the WadeGiles romanization of Fung Yu-lan will appear in some endnotes and some bibliographical entrics.

2. Zhongguo Zhexue Shi (Shanghai: Shen Zhou Publishing Company, 1931, Volume One only; reprinted Shanghai: Commercial Press, 1934, Volumes One and Two). Fung Yu-lan, A History of Chinese Philosophy, translated by Derk Bodde (Peiping: Henri Vetch, 1937, Volume One only; revised and reprinted Princeton, NJ: Princeton University Press, Volume On, 1952; Volume Two, 1953).

3. Feng Youlan's work in English was joined a decade later in 1963 by that of Wing-tsit Chan, A Source Book in Chinese Philosophy, also published by Princeton University Press.

4. This stepping forth from the known older order into unknown new challenges is neither new to nor limited to the twentieth century for China or for any traditional cultural heritage.

5. Three recent efforts include those of Michel C. Masson, Philosophy and Tradition: The Interpretation of China's Philosophic Past, Fung Yu-lan 1939-1949 (Ricci Institute: Taipei, 1985); Wang Jianping, Feng Youlan: Zhexue Sixiang Yanjui (Feng Youlan: Research into His Philosophical 
Thought) (Sichuan: Renmin Chubanshe, 1987 [in Chincse]); and Yin Ding (Yin Lujun), Fung Yu-lan (Taipei: Tung-ta Publishing Company, a division of Sanmin Publishing Company, 1991 [in Chinese]).

6. From September to January 31, 1988-89, the Editor taught in the Philosophy Department at Beijing University; she returned in April 1989 to continue that teaching and left June 7 after the tragic events of Tiananmen Square. During these months she twice met Professor Feng Youlan at his home at Beijing University. The return in December 1990 was her second return to Beijing for academic conferences after June 1989.

7. Zong Pu, or Feng Zhongpu, Feng Youlan's daughter, a well-known author of fiction in China, is a member of the Chinese Academy of Social Sciences, of the Institute for Research in Forcign Cultures, and of the Socicty of Chinese Writers.

8. The International Academy of Chinese Culture (Zhongguo Wenhuo Shuyuan) is a scholarly educational organization whose members desire to rejuvenate and to carry forward the positive aspects of Chinese culture and to reintroduce traditional culture in modern China through this academic organization. With this common desire, the International Academy was established in October 1984 by the renowned scholar Liang Shuming (1893-1988), along with Beijing University Professors Feng Youlan, Zhang Dainian, Zhou Yiliang, Tang Yijie, Yin Falu, Zhu Bokun, and the former President of the Institute of World Religion of the Chinesc Academy of Social Sciences, Professor Ren Jiyu, who is now Director of the Chinese National Library, and other scholars. With Liang Shuming as Chairman of the Administrative Committee, Feng Youlan served as Honorary President of the Academy. Today, Professor Zhang Dainian holds the latter distinguished position. Professor Tang Yijie from the beginning has been President of the International Academy. Professor Tang is assisted in the organizational and administrative tasks of the International Academy by Beijing University Department of Philosophy Professors Wang Shouchang and Li Zhonghua.

9. The two exceptions in this volume are the papers of Mr. Thanh Van Tran and Assistant Professor Yin Lujun, a mainland Chinese scholar currently teaching Comparative Ethics at Louisiana State University, Department of Philosophy and Religious Studies, who was unable to attend the conference but remains in close communication with philosophical work done at Beijing 
University.

10. Mr. Thanh Van Tran's cssay and that of Assistant Professor Yin Lujun were submitted in English.

11. Sce note \#104 on the Sony-Ming understanding of li as "pattern, principle."

12. The Editor realizes that this Western approach is somewhat more difficult for writers of Chinese. Were a Chinese writer to present a Western philosophical term in its original language first, followed by suggested Chinese matches with it, different typefaces would be required, one for alphabetic languages and one for Chinese characters. By contrast, only one typeface is required when romanizations of Chinese characters are placed in a Western language text as in this volume. Nonctheless, writers of English do include Chinese characters, often written by hand, in their English text. Similarly, some Chinese scholars write out Western philosophical terms by hand in their otherwise Chinese character text. This method works well when the Chinese text is running horizontally left to right. It obviously becomes more difficult when Chinese text is running vertically.

13. Sansongtang Zixu (Autobiography at the Hall of Threc Pines) (Beijing: Sanlian Shudian, 1984), p. 251. We shall have more to say on Chinese "you" (have, having) and Chinese "cunzai" (exist, existence) in Introduction, Part Three.

14. Tu Youguang is currently Professor of Philosophy at the Research Institute for Higher Education at Huazhong Ligong University in Wuchang, Sichuan. He is a graduate of National Qinghua University, Foreign Studies Academy, Department of Philosophy, where he studied under Feng Youlan. As Gencral Editor of Feng Youlan's Sansongtang Quanii, Tu has often been called affectionately Feng Youlan's "Number One Student."

15. Professor Li Zhonghua of the Beijing University Philosophy Department was with me.

16. For a recent discussion of May 4, 1919 events and their impact on events of May-June 1989, see Julia Ching, Probing China's Soul: Religion, Politics and Protest in the People's Republic (San Francisco: Harper and Row, 1990), Chapter Five.

17. For rich, in-depth narration of Chinese scholars who engaged in the "intellectual enlightenment" of the May Fourth period, see Vera Schwarcz, The Chinese Enlightenment (Berkeley, CA: University of California Press, 1986) 
and her brilliantly literary Time for Telling Truth is Running Out: Conversations with Zhang Shenfu (New Haven, CN: Yale University Press, 1992). To follow the continuing track of intellectual dissent in China from the 1940's to the present day, see Merle Goldman's carefully informative trilogy in sequential order: Literary Dissent in Communist China (Cambridge, MA Harvard University Press, 1967), China's Intellectuals: Advise and Dissent (Harvard University Press, 1981, and Sowing the Seeds of Democracy in China: Political Reform in the Deng Xiooping Era (Harvard University Press, 1994).

18. Chen Duxiu, Dean of Humanities at Beijing University, supporter of the May Fourth 1919 demonstrations, and later one of the founders of the Chinese Communist Party summarized aspirations of the period: 1) to oppose Japanese imperialistic aggression and corrupt Chinese rulers; 2) to oppose feudalism and its old ethics while promoting emancipation of thought and women, 3) to put an end to superstition while promoting science and industry, 4) to oppose the usc of clasșical written language in instruction and to promote the use of vernacular language and literature in education and in spreading culture to the people, and 5 ) to promote the rights of the people while opposing bureaucracy. This list is taken from Roderick MacFarguhar, The Hundred Flowers (London: Atlantic Books, 1960), p. 20 cited in Julia Ching, Probing China's Soul: Religion, Politics and Protest in the People's Republic (San Francisco: Harper and Row, 1990), Chapter Five, pp. 109, 253.

19. See Vera Schwarcz, Time for Telling Truth Is Running Out, op. cit., p. 97, for details of the "lost," off-the-public-record, beginning of the Chinese Communist Party with Chen Duxiu in Shanghai and Li Dazhao and Zhang Shenfu in Beijing.

20. Although different in content, perhaps in form and in accomplishment, this twentieth century, single-voiced allegiance in some ways has not been altogether different from single-voiced allegiances of China's past.

21. On Yan Fu, see Benjamin Schwartz, In Search of Wealth and Power: Yen Fu and the West (Cambridge, MA: The Belknap Press of Harvard University Press, 1964). On Kang Youwei, Tan Sitong, Zhang Binglin, and Liu Shipei, see Hao Chang, Chinese Intellectuals in Crisis: Search for Order and Meaning, 1890.1911 (Berkeley, CA: University of California Press, 1987). 
22. Liang Shuming (1893-1988) was Professor of Philosophy at Beijing University from 1918 to 1924. As one devoted to China's culture and people, Liang gave his life to rural reconstruction and Confucian modernization, while ever remaining a Buddhist in inner spiritual cultivation. In line with these endeavors, several decades later in the carly 1980s, Liang Shuming was the renowned founder of The International Academy for Chinese Culture (Zhongguo Wenhua Shuyuan) which hosted the "International Seminar on the Thought of Feng Youlan". in December 1990. For reference on Liang Shuming, the May Fourth Incident, and the "cultures controversy" as it continued to develop in the early 1920s, sce Guy Alitto, The Last Confucian: Liang Shu-ming and the Chinese Dilemma of Modernity (Berkeley: University of California Press, 1979, 1986), Chapter Three.

Regarding Liang Shuming's lectures on Eastern and Western culture, Alitto writcs:

Liang Shu-ming's statement on the subject, which was first published in book form at the end of 1921, was regarded by intellectuals at the time, and by historians since, as the link between Liang $\mathrm{Ch}$ ich'uo's [Liang Qichao] 1919 articles [which reported "to his countrymen that the West was crying for Eastern spiritual solace"] and Chang Chun Mai's [Zhang Junmai; also known as Carsun Chang in the West] "Philosophy of life" lecture [which raised "doubts about following the West's path of industrialism, capitalism and scientism"]. The three stand together in most people's minds as a wave of conservative backlash against the successes of the New Culture movement. In their anti-positivist bent their search for the core significance of Chinese culture under the encrustations of traditions, and their distaste for the modern industrialized West, the three do share common themes and approaches. (Ibid., pp. 77-8)

Influencing debates on Eastern and Western cultures were Columbia University's John Dewey, who lectured at Beijing University for a good part of the academic year 1919-1920 and spoke "of a future blending of Eastern and Western thought" and Bertrand Russell, who, while in residence at Beijing University in 1921, lectured in the direction "that China would somehow absorb Western science and technology while retaining the ethical qualities 
and humanistic way of life at which China was superior." (Ibid., pp. 76-77)

23. Feng Youlan, Zhongguo Zhexue Shi (Shanghai: Shen Zhou Publishing Company, 1931, Volume One only; reprinted Shanghai: Commercial Press, 1934, Volumes One and Two). Republished: Beijing: Zhonghua Shuju, 1961. Fung Yu-lan, A History of Chinese Philosophy, English translation by Derk Bodde (Peiping: Henri Vetch, 1937, Volume One only, revised and reprinted Princeton, NJ: Princeton University Press, Volume One 1952; Volume Two 1953).

24. Chinese "Hanxue" (Han Learning) was a discipline within Ru (Confucian) learning. Hanxue bcgan in the late Ming and carly Qing period and, over a period of two hundred years, developed mcthods of empirical research, using philological and phonological evidence among either types of empirical evidence, in an effort to recover "the original texts" of carly Ru (Confucian) scholarship. Thereby, Hanxue aspired to recover the original meaning of these texts. The impetus for the school may well have been to seck authoritative answers from texts of the "sages" themselves to questions posed by rivaling schools of Song-Ming $R u$ (Confucian) study.

25. A Short History of Chinese Philosophy, (New York: Macmillan, 1946), p. 333.

26. Ibid.

27. Ibid., p. 334. See Chapter Five of this volume, note \#2, for further details of these volumes completed by Feng Youlan, Tang Yongtong, and Jin Yuelin.

28. See Chapter One, note \#5, and Chapter Six, initial paragraph, for a discussion of the meaning of Feng's title: Zhen Yuan Liu Shu.

29. For reference, see also Professor Tu Youguang's essay in which he cites from Feng's $X$ in $Z h i$ Yan to the effect that the statement "some event/thing exists" (shiwh cunzai) is an affirmation concerning actuality, that is, a statement of fact. From this one statement of fact, New Lixue proceeds formally and analytically, without any further statements of fact, to deduce an entire metaphysical system.

30. For a descriptive historical account of these events, see Jonathan D. Spence, The Search for Modern China, (New York: W. W. Norton \& Company), Chapter 18.

31. Ibid., pp. 5634 .

32. Merle Goldman, "The Party and the Intellectuals," in The Cambridge History 
of China (Cambridge: Cambridge University Press, 1987), Volume Fourteen, Chapter Five, p. 234.

33. See the Editor's "Ministers of the Moral Order: Innovations of the Early Zhou Kings, the Duke of Zhou, Confucius, and $R u$," unpublished doctoral dissertation, Harvard University, 1984 which is under revision for publication. Sec also Merle Goldman, $o p$. cit., pp. 218-220.

34. Merle Goldman, "The Party and the Intellectuals," op. cit., p. 219.

35. Fur a general history of these campaigns from the 1930s to 1965, which sought to bring intellectuals under tight ideological and political control, sec Merlc Goldman, "The Party and the Intellectuals" and "The Party and the Intcllectuals: Phase Two," in The Cambridgc History of Ching, op. cit., Volume Fourtecn, Chapters Five and Ten, respectively.

36. For accounts of events in Feng Youlan's life, see Yin Ding (Yin Lujun), Fung Yulan, op. cit., pp. 205-234, and Wang Jianping, Feng Youlan, Zhexue Sixiang Yanjui, op. cit., pp. 249-283.

37. Through a careful program of initial solidarity, moving to isolation and guilt, fear and insecurity, then to resolution and fuller integration into the work of the Party, scholars were remoulded. See Jonathan D. Spence, op. cit., pp. 564-5, for exam ples.

38. ling Youlan gave self-criticisms ( $z i$ wo piping), beginning in 1950 with a sclf-criticism of his 1940s New Lixue system, entitled "Xin Lixue de Ziwo Jiantan" ("Sclf-criticism of Ncw Lixue"), Guangming Riboo, October 8, 1950. Feng's sclf-criticisms continued throughout the 1950s, more intensively in 1958 in the Anti-Rightist campaign following the Hundred Flowers Campaign. His most well-known self-criticism is Sishi Nian de Huigu (Looking Back Over Forty Years) (Beijing: Kexuc Chubanshe, 1959); also printed in scrics in Philosophical Research, Volume 3. This has been reprinted in Feng Youlan de Daolu (The Path of Feng Youlan) (Hong Kong: Panu Zazhi, 1974). See also an English translation, "Reflections on the Past Forty Years by Feng Youlan," by William A. Wycoff, Guest Editor, Chinese Studies in Philosophy, Vol. XIII, Nos. 2-3, 1981-2, pp. 9-126. See also Zhang Dainian's essay, Chapter Six of this volume, for excerpts from Feng's Sishi Nian de Huigu. Kam Louie, op. cit., pp. 52-53, offers an assessment of these 1958. 9 self-criticisms.

39. Hu Feng was a student of well-known May Fourth writer Lu Xun (18811936). On the Hu Feng campaign of 1955 , see Merle Goldman, "The Party 
and the Intellectuals," op. cit., pp. 239-242.

40. Regarding a rigt among leaders on whether to seek or not to seek intellectual support, Jonathan Spence writes:

Among the very senior members of the CCP (Chinese Communist Party) Politburo who favored an attempt to relax controls over the intellectuals, even if it meant allowing criticism of the CCP itself, were Mao Zedong, Zhou Enlai, the economic planner Chen Yun, the newly appointed secretary-general of the party Deng Xiaoping, and General Lin Biao. Among those insisting on tight party discipline, and on the belief that there was no longer a pressing need for alliance with bourgeois remnants, were two senior members of the Standing Committee of the Politburo, Liu Shaoqi and PLA commander in chief Zhu De, along with General Peng Dehuai and the tough party veteran who was now in the key position of mayor of Peking [Beijing], Peng Zhen.

Jonathan D. Spence, op. cit., p. 567.

41. See Communist China, 1955-59: Policy Documents with Analysis (Cambridge, MA: Harvard University Press, 1962), pp. 151-163; cited in Kam Louie, Inheriting Tradition: Intepretations of the Classical Philosophers in Communist China 1949.1966 (Oxford: Oxford University Press, 1986), p. 6 , note \#20.

The expression "hundred schools" describes scholars of the Warring States (480 B.C. -222 B.C.) period, who presented their views to feudal lords in power as regards how to order and to harmonize the kingdom and people. If the feudal lord were favorably persuaded, a scholar's approach would be implemented, at least to some extent, with the scholar receiving stipend and emolument as a Tutor. The parallels between this ancient period and the late-fifties period, after the break from the Soviet Union, are in the initial purpose of the latter campaign: namely, for those at the center of power to hear from scholars and, via these scholars, the people, as to how well the Communist Party was succeeding and what could be done better.

42. Merle Goldman, "The Party and the Intellectuals," op. cit., p. 243.

43. Jonathan D. Spence, op. cit., p. 567-570. Merle Goldman, "The Party and the Intellectuals,"op. cit., p. 248.

44. Jonathan D. Spence, op. cit., p. 569. 
45. Merle Goldman, "The Party and the Intellectuals," op. cit., p. 250.

46. Kam Louie, op. cit., pp. 33-41.

47. The issues and points of view articulated at this January 1957 conference were to influence philosophical discussion for the next two decades in China. A collection of important papers of the conference was published in the same year: Zhexue Yanjui Bianjibu (ed.), Zhongguo Zhexueshi Wenti Toolun Zhuanji (A Symposium on the Problems of the History of Chinese Philosophy) (Beijing, Kexue Chubanshe, 1957); cited in Kam Louje, op. cit., p. 258.

48. Kam Louie, op. cit., pp. 4, 34 .

49. See Chapter Five of this volume note \#2.

50. Kam Louie, op. cit., p. 36.

51. "Guanyu Zhongguo Zhexueshi Yanjiu de Liangge Wenti" ("Two Problems Concerning the Study of the History of Chinese Philosophy"), Renmin Ribao (People's Daily), October 23, 1956.

52. Kam Louie, op, cit., p. 41.

53. "Zhongguo Zhexue Yichan de Jicheng Wenti" ("On the Question of Inheriting the Legacy of Chinese Philosophy"), Guangming Ribao, January 8, 1957.

54. Kam Louie, op. cit., p. 44.

55. For a discussion of the January 1957 conference and especially Feng Youlan's presentations at the conference along with follow-up debates, see Kam Louie, op. cit., pp. 4147.

56. Jonathan D. Spence, op. cit., p. 570.

57. See Kam Louie, op. cit., p. 8; Jonathan D. Spence, op. cit., pp. 570-571; and Julia Ching, op. cit., pp. 110-113.

58. Jonathan D. Spence, op. cit., p. 572.

59. Ibid.:

60. Merle Goldman, "The Party and the Intellectuals," op. cit., pp. 255-8.

61. Kam Louie, op. cit., p. 48.

62. Ibid., p. 50.

63. Jbid., p. 54 .

64. During these debates, Guan Feng would continue to criticize Feng for inheriting Zhuang Zi's false views. Feng's spiritual realm of Heaven and Earth (Tian $D i$ ) was viewed as a direct inheritance of Zhuang $\mathrm{Zi}$ 's "Carefree Wandering" sort of thinking. In Guan Feng's view, such false thinking was detrimental to the social progress of China in that it led to escapist, negative 
tendencies and passive submission. Kam Louie, op. cit., pp. 111-112, 118-21. It is questionable whether Feng Youlan construed or used Zhuang Zi's views in the manner in which Guan Feng alleged he had, although sce ibid., p. 192. The Afterword of this volume presents further discussion of Feng Youlan and his views of Zhuang $\mathrm{Zi}$ interpreted through the Zhuang $Z i Z h u$ (Xiang Xiu-Guo Xiang Commentary on the Zhuang Zi).

65. See Afterword of this volume on this point.

66. See Kam Louie, op. cit., p. 123. Kam Louie notes that Feng Youlan's argument here had obvious implications for Feng Youlan himself in the early 1960 s.

67. See above note \#53.

68. Kam Louic notes that Feng Youlan, soon after presenting his January 1957 paper, was ready to substitute the term "general" for "abstract" and the term "specific" tor "concrete." In other words, Feng would change the terminology to save the method. See Kam Louic, op. cit., p. 45.

69. Sce Chapter One of this volume for a presentation of this, the first line of Feng Youlan's famous couplet.

70. Chapters Six, Nine, and Twelve of this volume offer further detail on the question of whether $l i$ (pattern, principle) is prior to shi (events) or whether $l i$ is within shi. One way of understanding the latter position is this: general principles are within events from age to age. This is different from imposing principles upon events from without or denying the presence of principle altogether.

71. "Lun Kong Zi Guanyu 'Ren' de Sixiang" ("Kong Zi On Thinking About Benevolence"), in Zhexuc Yanjiu (Philosophical Research), May 1961.

72. Karl Marx and Frederick Engels, "The German Ideology," in Selected Works (Moscow: Progress Publishers, 1960-70), Volume 1, p. 48; cited in Kam Louie, $o p$. cit., p. 55. Feng's citation from Marx brought considerable criticism in that it would appear that Feng quoted Marx out of context. Marx's overail point was that a ruling class imagines its values as universal.

73. For discussion of Feng Youlan's views on Kong $\mathrm{Zi}$ along with those of other scholars in the early 1970s, see Kam Louie, Critiques of Confucius in Contemporary' China (Hong Kong, Chinese University Press, 1980).

74. See Selected Bibliography for dates of publication for each volume of Feng Youlan's Zhongguo Zhexue Shi Xinbian (New Edition of A History of Chinese 
Philosophy). Volume Seven was published post-humously.

75. See Selected Bibliography for a listing of the volumes and publication dates for Feng Youlan's Sansongtang Quanji (The Collected Works at the Hall of Three Pines or The Collected Works of Feng Youlan), edited by Tu Youguang.

76. This is the second line of the couplet which serves as the title of the documentary film shown at the start of "The International Seminar on the Thought of Feng Youlan" on December 4, 1990. It is also the theme which runs through the whole of Feng's Xin Yuan Dao (The Spirit of Chinese Philosophy).

77. Professor Li's modern New Ruxue encompasses, perhaps, Professor Zhang Liwen's three modern versions of Song-Ming Lixue discussed above.

78. The Editor owes a considerable debt of gratitude to Professor Chen Lai, who in his essay in this volume carefully examines significant terms of SongMing Lixue and explains how Feng Youlan's New Lixue use of these terms differs from earlier Song-Ming Lixue use. Translation of Professor Chen Lai's essay required a thorough re-thinking of customary translations of Chinese "you" (have, having) and "zai" (is in, is present in) and taking a more literal rather than notional approach to translation. We have adopted Professor Chen Laijs format in our focus on terms in Part Three of this Introduction. However, our discussion of Chinese philosophical terms here differs from that of Professor Chen Lai in that our purpose is to explain these terms in English for the sake of non-readers of Chincse as well as, possibly, readers of Chinesc. In so doing, we seek to provide orientation for what Professor Chen Lai and others fathom more deeply in their own analyses.

79. Angus C. Graham, Studies in Chinese Philosophy and Philosophical Literature (Singapore: The Institute of East Asian Philosophies, 1986), p. 357.

80. Ibid.,.p. 359.

81. Ibid.

82. Ibid., p. 329.

83. Ibid., p. 330 .

84. Ibid., p.331.

85. We have used the term "actualist" instead of the term "realist" to differentiate the Chinese case from the Platonic, which will allow us to compare them rather than to assume from the beginning that they are the same by using the same name for both. In addition, there is good reason in describing the Chinese case to prefer "actualist" to "realist," as will be set forth shortly. 
86. For a discussion of names and their referring function, see Kao Kung-Yi and Diane B. Obenchain "Kung-sun Lung's Chih Wu Lun and Semantics of Reference and Prediction," The Journal of Chinese Philosophy 2 (1975) pp. 285-324. As regards the function of naming in Chinese language use, the view expressed here in Part Three of this Introduction is gleaned from many sources, Western and Chinese, and has developed in thought for over twentyfive years. We find that some of our own conclusions at certain points intersect with conclusions of some of Chad Hansen's recent writings, although our explanations differ. On naming, see Chad Hansen, Language and Logic in Ancient China (Ann Arbor, Ml: The University of Michigan Press, 1983), especially pp. 31-37. (Subsequently Hansen 1983.) See also Chad Hansen, "Chinese Idcographs and Western Ideas," in The Journal of A sian Studies 52:2 (May 1993), pp. 373-399, especially pp. 393-4 wherc Hansen takes the meaning of a name to be its "intention to refer to" an event/thing in actuality. (Subsequently Hansen 1993.) Finally, see Chad Hansen, "Language in the Heart-Mind," in Understanding the Chinese Mind: The Philosophical Roots, edited by Robert E. Allinson (Hong Kong: Oxford University Press, 1989), pp. 75-123, which explores social and cultural aspects related to Chinese vicws of naming. (Subsequently Hansen 1989.)

87. While early on Chinese characters wcre constructed so as to image in a pattern of lines the visual patterns of events/things to which they refer, Chinese characters have now become systematized both by visual organization and by sound. Few characters used today are strictly "representational pictures" of patterns discerned in events/things in actuality with which patterns in characters are deemed to be in correspondence. Nonetheless, the fact that characters remain patterns of lines, however visually and phonctically organized, assists in conveying the discernment of coherence between patterns of heart-mind functioning and patteris in actual events/things. Notionally, a sense of alignment of pattern from heart-mind through name (and visual character) to event/thing endures even with simplified Chinese characters of today.

Chad Hansen has argued that Chinese characters as patterns of lines convey meaning in a manner different from that of Western alphabetic words; see especially Hansen 1993. While we would agree with Hansen's observation, we would argue the case somewhat differently. See my forthcoming study on 
$R u$ (Confucians), op. cit., for more on this topic.

88. In Hansen's view, a name refers to "mass-stuff-kind." "Mass-stuff $k$ ind" differs from "count-kind" or "category/class." The expression "mass-stuffkind" suggests notionally one continuous stuff referred to by Chinese nouns which are both singular and plural or "mass nouns." Somewhat differently, the expression "count-kind" suggests that there are instances of a kind which can be counted and numbered, which are referred to by count nouns of Western languages that take pluralization. Hansen argues that this Western linguistic characteristic of count nouns encourages a philosophical discernment that there is one ideal or "universal" form or pattern which the mind knows, actual events/things, called "particulars," instantiate this universal form in more than one instance, but always with some variation and never perfectly or exactly. See Hansen 1983, 1993.

89. Chad Hansen contends that the view that one name corresponds to one actual event/thing comes from a teaching of the Warring States period called "rectification of names" or "correction of names" (zheng ming). In Hansen's view, this teaching belonged primarily to the $R u$ (Confucians); Gong-sun Long also subscribed to this view. See Hansen 1983, pp. 106-110 and Hansen 1989, pp. 107-119.

While $R u$ (Confucians), specifically $X u n Z i$, made a very strong case for this teaching in the late Warring States period, it is not clear what version, if any, of this teaching on "rectification of names" actually was Kong Zi's (Confucius') own. The collected sayings (A nalects) of Kong $\mathrm{Zi}$ are those remembered by his students and their students, and may well have been influenced by teachings which came into sway after Kong Zi's demise. Nor is it certain that the teaching originated with the $R u$ (Confucians). On this point, see A. C. Graham Disputers of the Tao (La Salle, IL: Open Court, 1989), p. 24, and Graham's survey of others' views. See also Benjamin I. Schwartz, The World of Thought in Ancient China, (Cambridge, MA: The Belknap Press of Harvard University Press, 1985), pp. 92-95, 311, 314.

90. A combined name, in some manner, joins or interconnects discriminative patterns in heart-mind, associated with each specific namc. Debates on how names combine, and how a combined name corresponds to one specific event/thing, intensified in the late Warring States period among the Later Mohists and others such as Gong sun Long, Hui Shi, and Zhuang $\mathrm{Zi}$, and 
continue today. Such debates concern 1) what thinking (si) is, 2) logical analysis of the meaning ( $y i)$ of names (ming), and 3) theories of inborn nature (xing) of actual events/things. Importantly in this regard, pattern (whether called doo. Tian, li) is discerned as in thinking, in naming, and in inborn nature.

On Later Mohist and Gong-sun Long debates, see Chad Hansen 1983, Chapters Four and Five. Sec also A. C. Graham, "Threc Studies of Kung-sun Lung," in his Studies in Chinese Philosophy and Philosophical Literature (Singapore: Institute of East A sjan Philosophies, 1986), pp. 125-215. And again, on the use of reasoning in Gong sun Long, the Later Mohists, Hui Shi and Zhuang $\mathrm{Zi}$, see A. C. Graham, Disputers of the Tao, op. cit., Part I, Chapter Five. Part II, Chapters Two and Three.

91. Sec A. C. Graham, Two Chinese Philosophers, (London: Lund Humphrics, 1958), Chapter on "Li" (principle).

92. On Chinese philosophy's not making a clear distinction between descriptive luws of nature and pescriptive rules of morality, between is and ought, fact and value, sce David Nivison, "Tao and Tc," in The Encyclopedia of Religion, edited by Mircea Eliade (New York: Macmillan Publishing company), Vol. 9. pp. 284-6, and A. C. Graham, Disputers of the Too, op. cit., pp. 29-30, 355-6. See also Hansen 1989, pp. 84-95, for a discussion of Chinese discourse as prescriptive.

93. See P. F. Strawson's view that "the meaning of an expression is the habits, conventions, rules for correct use of an expression, on all occasions, to refer or to assert" in P. F. Strawson. Logico-Linguistic Papers (London: Methuen \& Co., Ltd., 1971), p. 9; cited in Kao Kung-yi and Diane B. Obenchain "Kungsun Lung's Chih Wu Lun and Semantics of reference and Predication,"op. cit., p. 285.

94. On Chinese "shi-fei" use, sec also Angus C. Graham, Studies in Chinese Philo. sophy and Philosophical Literature, op. cit., pp. 331-343. See also Hansen 1989, pp. 99-102.

95. Sce Hansen 1989, pp. 75-123 in its entirety for a multi-approached account of just this theme; see, in particular, pp. 84-95. See also Hansen 1993, p. 393, and Hansen 1983, p. 105, for summaries of his views on this issue.

96. Languages of any sort function somewhat in the same manner as Chinese language does as far as names referring to the actual world is concerned. 
How ever, if, due to visual Chinese characters in patterns of lines, patterns of discrimination in the heart-mind are experienced as in tighter cohesion with patterns discerned in actual events/things than in other languages, then certain types of social control through thought or naming campaigns may be possible using the Chinese spoken and written language. However, one wonders whether another variable may be operative. For any language, "traditionalist" one-voiced, authoritative use of a language differs from "modernist" manyvoiced, elective use of a language.

97. The imposition of human-made pattern upon events/things is characteristic of the Chinese Legalist $(\mathrm{Fa} / \mathrm{ia})$ point of view.

98. This was a favorite expression of my father as my sisters and I grew up polishing wood.

99. This is the meaning of the second line in Feng Youlan's famous couplet which serves as the title of the documentary film on Feng Youlan's life discussed in Chapter One of this volume.

100. See Kan Kung-yi and Diane B. Obenchain, 'Kung-sun Lung's Chih Wu Lun and Semantics of Reference and Predication," op. cit., for a discussion of Chinese ergative semantics and case or role order syntax.

101. This linguistic focus on action is surcly correlated with Chinese philosophy's focus on action.

102. One hears a literal English translation of Chincse you, i.c., "have," as a fully meaningful sentence used all over Singapore these days. Everywhere on the street Chinese speakers of English can be heard to respond to any question such as "Do you have any new spapers?" with "Have, have" (in English).

103. On English "to be" as a "stop-gap" verb, see Graham, "Being in Western Philosophy Compared with Shih/Fei and $Y u / W u$ in Chinese Philosophy," in Graham, Studies in Chinese Philosophy and Philosophical Literature, op. cit., p. 330.

104. A C. Graham in his study of the two Cheng brothers, Cheng Yi (1033-1107) and Cheng Hao (1032-1085), who preceeded $\mathrm{Zhu} \mathrm{Xi} \mathrm{(1130-1200)} \mathrm{and} \mathrm{offered}$ much to the latter's metaphysical system, suggests that we picture $l i$ as "veins" in a piece of jade or in a body. The great innovation of the Cheng brothers, according to Graham, was their affirmation that "the innumerable $l i$ are one li." (See A. C. Graham, Two Chinese Philosophers, [London: Lund Humphries, 1958], p. 11.) We extend our knowledge of $l i$ by tracking with $l i$ 
to discover where one specific li connects with another, and this to another, until the whole of $l i$, the interconnecting web or pattern of $l i$, is discerned, cognized, and known. Graham states:

Principle [ii] scems to be conceived as a network of veins; however much they diverge from each other, the veins prove when we 'extend' them to be one; on the other hand we can also go on indefinitely making finer and finer distinctions among them, finding as we proceed that not only classes but individuals and parts of individuals have $l i$ which distinquish them from each other. (Ibid., p. 13.)

We are invited by $\mathrm{Zhu} \mathrm{Xi}$ to envision $l i$ as grain in wood:

There is only one principle (dooli), but its divisions are not the same...Thus there is only one for this board, but the grain runs one way here and another way there, one for a single house, but it has different sorts of rooms one for plants, but they include both peach and plum-trees; one for mankind, but there is Mr. A. and Mr.

B. Mr. A cannot become Mr. B; Mr. B cannot become Mr. A.

(Zhu Zi Yulei [Conversations of Master Zhu, Arranged Topically], $6 / 3 \mathrm{~B}, 4 \mathrm{~A} / 3$, citcd in Graham, ibid, p. 13.)

Importantly, cach individual event/thing has $l i$. The $l i$ of an individual event/ thing is one distinct line in the grain of the wood, as it were. As Zhu $\mathrm{Xi}$ has put it:

As far as the things in the universe go, we can be certain that each has a reason why it is as it is and a rule to which it should conform. This is what is meant by principle $[l i]$.

(Zhu Xi's Daxue Huowen 15:3, cited in Danicl Gardner's translation in his Learning to be a Sage, [University of California Press, 1990], p. 90.)

Insofar as each individual event/thing has its own individual $l i, l i$ would seem to differ, at least in this aspect, from Plato's idea or Aristotle's eidos (form) as a universal. $L i$ is the action which is/ought to be performed by an individual event/thing so named that differentiates that individual, event/thing or person from other individuals events/things or persons. (See A.C. Graham's discussion of this point, ibid., p. 18.) Knowing li resembles knowing names (ming) in the teaching of the Warring States period called "rectification of names" 
according to which every event/thing or person has a specific name and a specific task to perform which corresponds to that name.

Through logical analysis, we discover more general $l i$ and more specific $l i$; there is $l i$ of being first son and $l i$ of being third son. However, while it is possible to logically abstract, as it were, what is shared in common among li of first, second, and third sons, in practice this abstraction has not been of much practical use, for the abstraction does not tell an individual what to do. If one is first son, one acts in accordance with $l i$ of first son which is specific for lirst son and further specified by individual family custom, generation, location, anf so forth. The $l i$ of one person is never precisely the same as that for another; nor is $l i$ exactly the same for individual inanimate event/things. Hence, to know what is shared in common by different $l i$ of first, second, and third sons could be called a universal or an idea, but it would not be of much use in informing one how to act. Appropriate right action, not universal ideas, has been what matters in Chinese society. Each individual event/thing has $l i$, an action which that individual is to perform. That action joins with other actions and these in turn interconnect with others, extending in all directions to unite the whole of action in one pattern li or dao, onc patterned activity of all actual events/things in our everyday under Heaven.

105. A. C. Grahan has pointed out that unlike $l i$, which is metaphorically illustrated by veins in stone or grain in wood, $q i$ is "the breath in our throats, ... the source of life, ... [we] feel it rising and ebbing in our bodies as physical energy, ...we smell it as odours" and much more. This is the subtle end of $q i$. The more concrete, gross side of $q i$ is matter (zhi or zhiliao). Graham states that by the Song Dynasty, solid things were viewed as condensing from and dissolving back into a more subtle $q i$, such that "matter (zhi) is mrely ether $[q i]$ in a very dense and inert state." See A.C. Graham, Two Chinese Philosophers, op. cit, p. 31.

106. A history of the modern development and use of the Chinese term "cunzai" would make an important contribution to Chinese-Western comparative philosophy.

107. Chung-wen ta-tz'u-tien (The Encyclopedic Dictionary of the Chinese Language), edited by Chang Ch'i-yun, Lin Yin, and Kao Ming (Taipei: Institute for Advanced Chinese Studies, 1962-68, reprinted edition, Taipei: Huakang Ch'u-pan-pu, 1974), Volume 3,pp. 279-282. 
108. We would agree with A. C. Graham that Chinese you is used most often of events/things in the actual world. However, we would disagree with Graham that other uses of you (and its negative $w u$ ) are only "occasional." Further, we would agree with Graham that "usually" dao or $l i$ are described as "wh (not having)," but would not agree with Graham's translation of "wu" as "Nothing" or "Void" in these contexts. See Angus C. Graharn, "Being' in Western Philosophy Compared with Shih/Fei and $Y u / W u$ in Chinese Philosophy," in Graham, Studics in Chinese Philosophy and Philosophical Litera. ture, op. cit., p. 344.

109. Chinese $l i$ (pattern, principle) can substitute for doo (way, path) in this expression.

110. Chinese "er" is a term which connects two expressions. It may be translated into English as "and" or "but," or the first expression may be taken as modify ing the second expression with "er" connecting them.

111. Analects, Book 5, Chapter 7 and 21, cited in Benjamin 1. Schwartz, The World of Thought in Ancient China, op. cit., p. 62. Sce Raymond Dawson's transiation, Confucius, The Analects, (Oxford: Oxford University Press, 1993), pp. 16, 18.

112. Zhang Dainian, Zhongguo Gudian Zhexue Gainian Fanchou Yao Lun (Discussion of the Essentials of the Conceptual Categories of Chinese Classical Philosophy) (Beijing, 1989), p. 79.

113. Graham, Studics in Chinese Philosophy and Philosophical Literature, op. cit., p. 345. Pinyin romanization added.

114. Ibid., loc cit. Pinyin romanization added.

115. Ibid., p. 346. Pinyin romanization added.

116. Jbid., p. 347. Pinyin romanization added.

117. In Sansongteng $Z i x u$ (Autobiography at the Hall of Three Pines) op. cit., p. 251 , Feng states:

In fact, "to have" (you) is "to exist" (cunzai). If ["to have"] is not "to exist," then "to have" has no meaning. (Qishi 'you" jiushi "cunzai." Ruguo bushi "cunzai," "you" ye iiu meiyou shenma yiyile.)

118. See Chapter Seven in this volume, the essay by Japanese scholar Azuma Juji, entitled "The Formation of New Lixue: Feng Youlan and New Realism."

119. This description of "subsistence" follows Webster's New Twentieth Century Dictionary of the English Language (unabridged) (Cleveland and New York: 
The World Publishing Company, 1966), pp. 897 and 1817.

120. The term li (pattern, principle) may substitute in these assertions here as well.

121. Wc shall have more to say about English translations of "shiji" and "zhenji" below.

122. Seng Zhao (384-414) in the third chapter of his book Banro Wuzhi Lun (On Prajna Not Being Know ledge) writes:

Because Wisdom (is assumed to) know what is to be known and to apprehend the qualities (of things), it is said to be knowledge. But since Absolute Truth inherently lacks any phenomenal qualities, how is it to be "known"?

In discussing this passage in $A$ History of Chinese Philosophy, Feng states:

The qualities [xiang] of a thing are the answer to the question of what that thing is. To know what a thing is, is to apprehend the qualities of that thing. But since Absolute Truth is not a "thing," it lacks the qualitics of such and hence cannot be known by ordinary knowledge.

Sec Feng Youlan, A History of Chinese Philosophy, Vol. 2, p. 266, for discussion of and citation from Seng Zhao, Zhoo Lun (The Book of Zhao) translated by Walter Liebenthal, Monumento Serica, Monograph XIII, (Peiping: Catholic University of Peking, 1948), p. 80. (Pinyin romanization has been used for names and titles in this citation.)-editor]

123. Graham,op. cit., p. 345. Pinyin romanization added.

124. The term "docetism" is derived from the Greek word meaning "to seem" and has been applied in the history of Christian thought to the vicw that the humanity and suffering of Jesus Christ was only "apparent" and not in fact. The term could be used to describe Feng's discernment of the relationship of $l i$ to events/things. Whereas in Song-Ming $R u$ discernment, substance is discerned as actually engaging in function, in Feng Youlan's discernment, substance is not discerned as actually engaging in function. Rather, substance is discerned as in the world of truth. Function is discerned as in the world of actual events/things. Feng does not discern any direct connection between substance and function. While for Feng some connection between the two obtains, it is not direct or actual, but, rather, apparent or manifest. For this reason, we have suggested the term "docetic" to describe Feng's view.

125. Bertrand Russell, The Problems of Philosophy (London, New York: Oxford University Press, 1912), Chapter Nine, "The World of Universals." The sen- 
tence presented here is taken from our paraphrased translation of a point made by Professor Azuma Juji in his essay, Chapter Seven of this volume.

126. See Section One of Professor Chen Lai's essay, Chapter Nine of this volume.

127. Professor Chen Lai will present in his essay that " $j i$ " has the sense of "cunzai" (existence).

128. A. C. Graham describes "is" used as a connective verb as a "stop gap" verb, supplied only becausc English requires that a sentence have a verb. Sce A.C. Graham, "Being" in Western Philosophy Compared with Shih/Fei and $Y u / W u$ in Chinese Philosophy," op. cit., p. 330.

129. See A. C. Graham's discussion of "wei" (to do, to make, to act) in Ibid., p. 326.

cxii 